NBER WORKING PAPER SERIES

AI AND JOBS: EVIDENCE FROM ONLINE VACANCIES

\author{
Daron Acemoglu \\ David Autor \\ Jonathon Hazell \\ Pascual Restrepo \\ Working Paper 28257 \\ http://www.nber.org/papers/w28257 \\ NATIONAL BUREAU OF ECONOMIC RESEARCH \\ 1050 Massachusetts Avenue \\ Cambridge, MA 02138 \\ December 2020, Revised February 2022
}

We thank Bledi Taska for detailed comments and providing access to Burning Glass data; Joshua Angrist, Andreas Mueller, Rob Seamans, and Betsey Stevenson for very useful comments and suggestions; Jose Velarde and Zhe Fredric Kong for expert research assistance; and David Deming and Kadeem Noray for sharing their code and data. Acemoglu and Autor acknowledge support from Accenture LLP, IBM Global Universities, Schmidt Futures, and the Smith Richardson Foundation. Acemoglu acknowledges support from Google, the National Science Foundation, the Sloan Foundation, and the Toulouse Network on Information Technology, and Autor thanks the Carnegie Fellows Program, the Heinz Family Foundation, and the Washington Center for Equitable Growth. The views expressed herein are those of the authors and do not necessarily reflect the views of the National Bureau of Economic Research.

NBER working papers are circulated for discussion and comment purposes. They have not been peer-reviewed or been subject to the review by the NBER Board of Directors that accompanies official NBER publications.

(C) 2020 by Daron Acemoglu, David Autor, Jonathon Hazell, and Pascual Restrepo. All rights reserved. Short sections of text, not to exceed two paragraphs, may be quoted without explicit permission provided that full credit, including $\odot$ notice, is given to the source. 
AI and Jobs: Evidence from Online Vacancies

Daron Acemoglu, David Autor, Jonathon Hazell, and Pascual Restrepo

NBER Working Paper No. 28257

December 2020, Revised February 2022

JEL No. J23,O33

\section{ABSTRACT}

We study the impact of AI on labor markets using establishment-level data on vacancies with detailed occupation and skill information comprising the near-universe of online vacancies in the US from 2010 onwards. There is rapid growth in AI related vacancies over 2010-2018 that is greater in AI-exposed establishments. AI-exposed establishments are reducing hiring in non-AI positions. We find no discernible relationship between AI exposure and employment or wage growth at the occupation or industry level, however, implying that AI is currently substituting for humans in a subset of tasks but it is not yet having detectable aggregate labor market consequences.

Daron Acemoglu

Department of Economics, E52-446

Massachusetts Institute of Technology

77 Massachusetts Avenue

Cambridge, MA 02139

and NBER

daron@mit.edu

David Autor

Department of Economics, E52-438

Massachusetts Institute of Technology

77 Massachusetts Avenue

Cambridge, MA 02139

and NBER

dautor@mit.edu
Jonathon Hazell

Department of Economics

London School of Economics

32 Lincoln's Inn Fields

United Kingdom

jadhazell@gmail.com

Pascual Restrepo

Department of Economics

Boston University

270 Bay State Road

Boston, MA 02215

and NBER

pascual@bu.edu 


\section{INTRODUCTION}

The last decade has witnessed rapid advances in artificial intelligence (AI) based on new machine learning techniques and the availability of massive data sets. ${ }^{1}$ This change is expected to accelerate in the years to come (e.g., Neapolitan and Jiang, 2018, Russell, 2019), and AI applications have already started to impact businesses (e.g., Agarwal, Gans and Goldfarb, 2019). Some commentators see this as a harbinger of a jobless future (e.g., Ford, 2015; West, 2018; Susskind, 2020), while others consider the oncoming AI revolution as enriching human productivity and work experience (e.g., McKinsey Global Institute, 2017). The persistence of these contrasting visions is unsurprising given the limited evidence to date on the labor market consequences of AI. Data collection efforts have only recently commenced to determine the prevalence of commercial AI use, and we lack systematic evidence even on whether there has been a major increase in AI adoption - as opposed to just extensive media coverage.

This paper studies AI adoption in the US and its implications. Our starting point is that AI adoption can be partially identified from the footprints it leaves at adopting establishments as they hire workers specializing in AI-related activities, such as supervised and unsupervised learning, natural language processing, machine translation, or image recognition. To put this idea into practice, we build an establishment-level data set of AI activity based on the near-universe of US online job vacancy postings and their detailed skill requirements from Burning Glass Technologies (Burning Glass or BG, hereafter) for the years 2007 and 2010 through $2018 .^{2}$

We start with a task-based perspective, linking the adoption of AI and its possible implications to the task structure of an establishment. This perspective emphasizes that current applications of AI are capable of performing specific tasks and predicts that firms engaged in those tasks will be the ones that adopt artificial intelligence technologies. ${ }^{3}$ To identify the tasks compatible with current AI technologies, we use three different but complementary

\footnotetext{
${ }^{1} \mathrm{AI}$ is a collection of algorithms that act intelligently by recognizing and responding to the environment to achieve specified goals. AI algorithms process, identify, and act upon patterns in unstructured data (for example, speech data, text, or images) to achieve specified goals.

${ }^{2}$ The BG data have been used in several recent papers. Alekseeva et al. (2021) and Babina et al. (2020), discussed below, use BG data to study AI use and its consequences. Papers using BG data to explore other questions include Hershbein and Kahn (2016), Azar et al. (2018), Modestino, Shoag, and Ballance (2019), Hazell and Taska (2019) and Deming and Noray (2020).

${ }^{3}$ See Acemoglu and Autor (2011) and Acemoglu and Restrepo (2018, 2019a). This is not the only possible approach to AI. One could also think of AI as complementing some business models (rather than performing specific tasks within those models) or as allowing firms to generate and commercialize new products (see Agarwal, Gans and Goldfarb, 2019, and Bresnahan 2019). We explain below why the task-based approach is particularly well-suited to our empirical approach and how it receives support from our findings.
} 
measures: Felten, Raj and Seamans' (2018, 2019) AI Occupational Impact measure; Brynjolfsson, Mitchell and Rock's (2018, 2019) Suitability for Machine Learning (SML) index; and Webb's (2020) AI Exposure score. These indices all identify sets of tasks and occupations that are most impacted by AI technologies, but each is computed based on different assumptions on AI capabilities. We construct an establishment's AI exposure from its baseline (2010-2012) occupational structure according to each one of these indices, and use these baseline measures as proxies for AI exposure throughout our analysis. ${ }^{4}$ Since our goal is to study the impact of AI on AI-using firms rather than AI-producing firms, we exclude firms in the professional and business services and information technology sectors (NAICS 51 and 54 ), both of which are primary suppliers of AI services.

Our first result is that there is a rapid takeoff in AI vacancy postings starting in 2010 and significantly accelerating around 2015-2016. Consistent with a task-based view of AI, this activity is driven by establishments with task structures that are compatible with current AI capabilities. For instance, a one standard deviation increase in our baseline measure of AI exposure based on Felten et al.- approximately the difference in the average AI exposure between finance and mining and oil extraction - is associated with $15 \%$ more AI vacancy posting. The strong association between AI exposure and subsequent AI activity is robust to numerous controls and specification checks when using the Felten et al. and the Webb measures, but this is less apparent with the SML index. This leads us to place greater emphasis on the Felten et al. and Webb measures when exploring the effects of AI exposure on the demand for different types of skills and non-AI hiring.

Our second result establishes a strong association between AI exposure and changes in the types of skills demanded by establishments. With the Felten et al. and Webb measures (and to a lesser extent, with SML), we find that AI exposure is associated with both a significant decline in some of the skills previously sought in posted vacancies and the emergence of new skills. This evidence bolsters the case that AI is altering the task structure of jobs, replacing some human-performed tasks while simultaneously generating new tasks accompanied by new skill demands.

The finding that establishments with AI-suitable tasks hire workers into AI positions and change their demand for certain types of skills does not, of course, tell us whether AI is increasing or reducing overall or non-AI hiring in exposed establishments. In principle, AI-exposed establishments may see an increase in (non-AI) hiring, if either AI directly

\footnotetext{
${ }^{4}$ Figure 4 below shows that the relationship between mean wage of an occupation and the three AI exposure measures is distinct, which is the basis of our claim that each one of these indices captures a different aspect of AI exposure.
} 
complements workers in some tasks, increasing their productivity and encouraging more hiring, or AI substitutes for workers in some tasks, but increases total factor productivity sufficiently to raise demand in non-automated tasks via a productivity effect (Acemoglu and Restrepo, 2019a). Alternatively, AI adoption may reduce hiring if AI technologies are replacing many tasks previously performed by workers and the additional hiring they spur in non-automated tasks does not make up for this displacement.

Our third main result shows that AI exposure is associated with lower (non-AI and overall) hiring. These results are robust in all of our specifications using the Felten et al. measure and in most specifications with the Webb measure, but, as anticipated, not with SML. The timing of these relationships is also plausible: substantial declines in hiring take place in the time window during which AI activity surged-between 2014 and 2018. This pattern of results, combined with the concentration of AI activity in more AI-exposed tasks, suggests that the recent AI surge is driven in part by the automation of some of the tasks formerly performed by labor. We find no evidence for either the view that there are major human-AI complementarities in these establishments or the expectation that AI will increase hiring because of its large productivity effects - though we cannot rule out that other applications of AI that are not captured here could have such effects.

In contrast to the establishment-level patterns, we do not detect any relationship between AI exposure and overall employment or wages at the industry or occupation level. There are no significant employment impacts on industries with greater exposure to AI, and also no employment or wages effects for occupations that are more exposed to AI. We conclude that despite the notable surge in AI adoption, the impact of this new technology is still too small relative to the scale of the US labor market to have had first-order impacts on employment patterns outside of AI hiring itself. Nevertheless, our main findings - that AI adoption is driven by establishments that have a task structure that is suitable for AI use and that this has been associated with significant declines in establishment hiring -imply that any positive productivity and complementarity effects from AI are at present small compared to its displacement consequences.

Our paper builds on Alan Krueger's seminal work on the effects of new digital technologies on workers and wages (Krueger, 1993; Autor, Katz and Krueger, 1998). Subsequent literature has investigated the implications of automation technologies, focusing on wages, employment polarization and wage inequality (e.g., Autor, Levy and Murnane, 2003; Goos and Manning, 2007; Autor and Dorn, 2013; Goos, Manning, and Salomons, 2014; Michaels, Natraj and Van Reenen, 2014; Gregory, Salomons and Zierahn, 2016). Recent work has studied the impact of specific automation technologies, especially industrial robots, on employment and wages, 
focusing either on industry-level variation (Graetz and Michaels, 2019), local labor market effects (Acemoglu and Restrepo, 2020a), or firm-level variation (Dinlersoz and Wolf, 2018; Bessen et al., 2019, Bonfiglioli et al., 2019; Dixon, Hong and Wu, 2019; Koch, Manuylov and Smolka, 2021; Humlum 2019 and Acemoglu, Lelarge and Restrepo, 2020).

There are fewer studies of the effects of AI specifically, though this body of work is growing rapidly. Bessen et al. (2018) conduct a survey of AI startups and find that about $75 \%$ of AI startups report their products help clients make better predictions, manage data better, or provide higher-quality. Only 50\% of startups report that their products help customers automate routine tasks and reduce labor costs. Grennan and Michaely (2019) study how AI algorithms have affected security analysts and find evidence of task substitution: analysts are more likely to leave the profession when they cover stocks for which there are abundant data available. Differently from these papers' focus on AI-producing sectors and specific applications of AI such as finance, we study this technology's effects on AI-using establishments and non-AI workers throughout the economy.

Most closely related to our paper are a few recent works also investigating the effects of AI on firm-level outcomes. Babina et al. (2020) study the relationship between AI adoption and employment and sales at both the firm and the industry level. They document that, consistent with Alekseeva et al. (2020), AI investment is stronger among firms with higher cash reserves, higher mark-ups and higher R\&D intensity, and moreover, these firms grow more than non-adopters. A contrast between our approach and Babina et al.'s is that we focus on AI suitability based on establishments' occupational structures rather than observed AI adoption, and this may explain why we arrive at different results for hiring. Also related is Deming and Noray (2020), who use Burning Glass data to study the relationship between wages, technical skills, and skills obsolescence. Though their focus is not AI, their work demonstrates that Burning Glass data are suitable for detecting changes in job skill requirements, an angle of inquiry we pursue below.

As noted above, our work exploits measures of AI-suitability developed by Felten, Raj and Seamans (2018, 2019), Brynjolfsson, Mitchell and Rock (2018, 2019), and Webb (2020). Our results are consistent with Felten, Raj and Seamans (2019), who find a positive relationship between AI-suitability and AI vacancy posting, but no relationship with employment growth, at the occupational level. We confirm that AI suitability is not at present associated with greater hiring in more highly-exposed occupations or industries, but we find robust effects on skill demand and a negative impact on establishment hiring.

The rest of the paper is organized as follows. Section 2 presents a model motivating our empirical strategy and interpretation. Section 3 describes the data, and Section 4 presents 
our empirical strategy. Section 5 presents our main results on AI exposure and AI hiring, while Section 6 looks at changes in the types of skills AI-exposed establishments are looking for. Section 7 explores the effects of AI on hiring at the establishment, industry and occupation levels. Section 8 concludes. Additional robustness checks and empirical results are presented in the Appendix.

\section{THEORY}

In this section, we provide a model that motivates our empirical approach and interpretation.

\subsection{Tasks, Algorithms and Production}

Establishment $e$ 's output, $y_{e}$, is produced by combining the services, $y_{e}(x)$, of tasks $x \in \mathcal{T}_{e} \subset$ $\mathcal{T}$ with unit elasticity (i.e., a Cobb-Douglas aggregator):

$$
\ln y_{e}=\ln A_{e}+\int_{\mathcal{T}_{e}} \alpha(x) \ln y_{e}(x) d x
$$

where $\mathcal{T}$ is the set of feasible tasks, a subset $\mathcal{T}_{e}$ of which is used in the production process of establishment $e$, and $\alpha(x) \geq 0$ designates the importance or quality of task $x$ in the production process and are common across establishments. We impose $\int_{\mathcal{T}_{e}} \alpha(x) d x=1$ for all feasible $\mathcal{T}_{e}$, which ensures that all establishments have constant returns to scale.

Establishments differ in their productivity term $A_{e}$, and, more importantly, in the set of tasks they perform (for example, because they produce different goods and services or use distinct production processes). We also assume that each establishment faces a downwardsloping demand curve for its product and will set its price $p_{e}$ to maximize profits (and its problem is separable from the profit-maximization problem of the firm's other establishments in case of multi-establishment firms). In this profit-maximization problem, we assume that each establishment is small in the labor market and takes other prices and aggregate output as given.

Tasks are produced by human labor, $\ell_{e}(x)$, or by services from AI-powered algorithms, $a_{e}(x)$ :

$$
y_{e}(x)=\left[\left(\gamma_{\ell}(x) \ell_{e}(x)\right)^{\frac{\sigma-1}{\sigma}}+\left(\gamma_{a}(x) a_{e}(x)\right)^{\frac{\sigma-1}{\sigma}}\right]^{\frac{\sigma}{\sigma-1}}
$$

where $\sigma$ is the elasticity of substitution between labor and algorithms, and $\gamma_{\ell}(x)$ and $\gamma_{a}(x)$ are assumed to be common across establishments. We assume that AI services are provided by combining AI capital (machinery or algorithms) purchased from the outside, $k_{e}(x)$, and in- 
house workers operating, programming, or maintaining this capital, $\ell_{e}^{A I}(x)$, with the following technology:

$$
a_{e}(x)=\min \left\{k_{e}(x), \ell_{e}^{A I}(x)\right\}
$$

which implies that in-house AI workers need to be combined with capital in fixed proportions. ${ }^{5}$ We assume throughout that all establishments are price takers for production workers, AI workers, and AI capital, whose respective prices are: $w, w^{A I}$, and $R$.

We view recent advances in AI as increasing the ability of algorithms to perform certain tasks - corresponding to an increase $\gamma_{a}(x)$ for some $x$. In what follows, we denote by $\mathcal{T}^{A}$ the subset of tasks that, due to these advances, can now be profitably performed by algorithms/AI. These advances in AI technology will have heterogeneous impacts on establishments depending on their task structure. For example, an increase in $\gamma_{a}(x)$ for text recognition will impact establishments in which workers perform significant text recognition tasks and will change the factor demands of these "exposed establishments".

To make these ideas precise, we define establishment $e$ 's exposure to AI as

$$
\text { Exposure to } \mathrm{AI}_{e}=\frac{\int_{x \in \mathcal{T}_{e} \cap \mathcal{T}^{A}} \ell_{e}(x) d x}{\int_{x \in \mathcal{T}_{e}} \ell_{e}(x) d x},
$$

where the employment shares are measured before the advances in AI take place. This measure represents the share of tasks performed in an establishment that can now be performed by AI-powered algorithms. ${ }^{6}$

We next explore how advances in AI impact AI activity and the demand for (non-AI) workers.

\subsection{Task Structure and AI Adoption}

To illustrate how the task structure determines AI adoption, we follow Acemoglu and Autor (2011) and Acemoglu and Restrepo (2018, 2019a) and assume that $\sigma=\infty$, so that algorithms

\footnotetext{
${ }^{5}$ This assumption can be relaxed in various ways. First, the technology can be more general than Leontief, so that factor prices affect how intensively AI workers are used. Second, establishments may be allowed to substitute outsourced AI workers for in-house services. The first modification would not have any major effect on our results, while the second would imply that our proxy for AI activity at the establishment level may understate the extent of AI, potentially leading to attenuation of our estimates.

The common technology assumption in (2) and (3) can also be relaxed but is useful for simplifying the exposition by ensuring that differences in factor demands across establishments are driven entirely by task structures, making the link between the model and our empirical approach more transparent.

${ }^{6}$ When $\sigma=\infty$ as in Propositions 1 and 2 below and the share of AI algorithms in cost is initially small, exposure to $\mathrm{AI}$ is $\int_{x \in \mathcal{T}_{e} \cap \mathcal{T}^{A}} \alpha(x) d x$, which gives the share of tasks that can now be completed with AI in total costs.
} 
and labor are perfectly substitutable within a task. We also focus on the realistic case in which the initial cost share of AI, denoted by $s_{e}^{A}\left(=\left(R k_{e}(x)+w^{A I} \ell_{e}^{A I}(x)\right) /\right.$ total costs $)$, is small. Additionally, we consider the problem of a single establishment, holding the prices of other establishments in the market as given.

Proposition 1 Suppose that $\sigma=\infty$ and the initial cost share of $A I, s_{e}^{A}$, is small. Consider an improvement in AI technologies that increases $\gamma_{a}(x)$ in $\mathcal{T}^{A}$ and leads to the use of AI algorithms in these tasks. Then the effects on the cost share of AI and in-house AI employment are given by

$$
d s_{e}^{A}=\text { Exposure to } A I_{e} \geq 0
$$

and

$$
d \ln \ell_{e}^{A I}=\left(\frac{1-s_{e}^{A}}{s_{e}^{A}}+\left(\varepsilon_{e} \cdot \rho_{e}-1\right) \cdot\left(1-s_{e}^{A}\right) \cdot \pi_{e}\right) \cdot \text { Exposure to } A I_{e} \geq 0,
$$

where $\varepsilon_{e}>1$ is the demand elasticity faced by the establishment, $\rho_{e}>0$ its passthrough rate, and $\pi_{e} \geq 0$ the average percent cost reduction in tasks performed by AI.

The proof of this proposition is provided in Appendix A, where we also provide the expressions for the passthrough rate, $\rho_{e}$, and average cost savings from the use of AI algorithms, $\pi_{e}$.

The proposition shows that changes in AI activity and hiring of AI workers are both proportional to exposure to AI. Motivated by these results, in our empirical work we use exposure to AI as the key right-hand side variable and identify greater use of AI with the posting of more vacancies for in-house AI workers.

Although in this proposition we focused on the case where $\sigma=\infty$, a similar logic applies when $\sigma>1$ and AI does not fully replace workers in the tasks it is used. In this case, AI advances still increase the cost share of AI and the hiring of AI workers in exposed establishments. When $\sigma<1$, however, technological advances will not raise the cost share of AI because of strong complementarities between tasks produced by algorithms and humans.

\subsection{AI, Task Displacement and Hiring}

The next proposition characterizes the effects of AI advances on hiring of (non-AI) workers. Its proof is also in Appendix A.

Proposition 2 Suppose that $\sigma=\infty$ and the initial AI share of costs, $s_{e}^{A}$, is small. Consider an improvement in AI technologies that increases $\gamma_{a}(x)$ in $\mathcal{T}^{A}$ and leads to the use of $A I$ 
algorithms in these tasks. The effects on on non-AI employment, $\ell_{e}$, are:

$$
d \ln \ell_{e}=\left(-1+\left(\varepsilon_{e} \cdot \rho_{e}-1\right) \cdot \pi_{e}\right) \cdot \text { Exposure to } A I_{e}
$$

where $\varepsilon_{e}>1$ is the demand elasticity faced by the establishment, $\rho_{e}>0$ its passthrough rate, and $\pi_{e} \geq 0$ the average percent cost reduction in tasks performed by $A I$.

Proposition 2 shows that the effects of AI advances on labor demand are proportional to our exposure measure. More centrally, it clarifies the effects of AI advances on labor demand. The direct consequence of such advances is to expand the set of tasks performed by algorithms, $\mathcal{T}^{A}$, and to shrink the set of tasks allocated to workers in exposed establishments. Because $\sigma=\infty$, this technological improvement displaces workers from tasks in $\mathcal{T}^{A}$. This displacement effect is captured by the " -1 " in the parentheses on the right-hand side of (5). In addition, as emphasized in Acemoglu and Restrepo (2018), the reallocation of tasks from workers to algorithms reduces costs and expands establishment output, $y_{e}$ (and this output response depends on the demand elasticity and the passthrough rate). This "productivity effect", the magnitude of which is proportional to the cost reductions due to AI, $\pi_{e} \geq 0$, increases hiring in non-automated tasks. If the second term on the right-hand side of (5), $\left(\varepsilon_{e} \cdot \rho_{e}-1\right) \cdot \pi_{e}$, exceeds -1 , the productivity effect dominates and AI technologies increase hiring. ${ }^{7}$ Otherwise, AI advances will reduce (non-AI) hiring in exposed establishments.

We make two additional remarks. First, as with the results on AI activity, the main conclusions of Proposition 2 can be generalized to the case in which $\sigma>1$. In this case, not all workers previously employed in AI-ex post tasks would be displaced, but the substitution away from them to algorithms would create a negative displacement and a positive productivity effect, similar to those in the proposition.

Second, if different tasks require different skills, then the adoption of AI technologies may also change the set of skills that exposed establishments demand (and list in their vacancies). Skills relevant for tasks now performed by algorithms will be demanded less frequently, and new skills necessary for working alongside AI algorithms may also start being included in vacancies.

Our empirical work will be based on equation (5). We will explore the relationship between AI exposure, as defined in (4), and changes in the number and skill content of the vacancies an establishment posts. Specifically, we will look at whether exposed establishments hire more AI workers, demand different sets of skills, and whether they increase or

\footnotetext{
${ }^{7}$ This expression also clarifies that when the passthrough rate is less than $1 / \varepsilon_{e}$, the establishment's price increases sufficiently that output does not expand and thus employment always declines.
} 
reduce their hiring of non-AI workers.

\subsection{Human-Complementary AI}

We have so far not considered human-complementary effects of AI. The possibility that AI will complement workers engaged in exposed tasks can be captured by assuming that $\alpha(x)$ increases for exposed tasks (see eq. 1) or, alternatively that $\sigma<1$, so that algorithms and human labor are complementary within a task (or both). This type of human-complementary AI may increase labor demand because algorithms raise human productivity in exactly the tasks in which AI is being adopted.

Evidence that AI is associated with greater establishment-level employment would be consistent with the human-complementary view, but could also be consistent with task substitution associated with large productivity gains that nonetheless increase hiring at exposed establishments. Conversely, evidence of negative, or even zero, effects would weigh against both the human-complementary view and the possibility of large productivity gains from AI - since both AI-human complementarity and large productivity effects boosting employment in non-automated tasks could generate a positive relationship between AI exposure and establishments hiring. Our evidence below finds negative effects of AI exposure on (non-AI) hiring, and thus suggests that the current generation of AI technologies is predominantly task-replacing and generates only modest productivity gains. ${ }^{8}$ It remains possible that other AI technologies than the ones we are proxying here could have different effects.

\subsection{Measuring Exposure to AI}

Propositions 1 and 2 show that we should see the effects of advances in AI in establishments with task structures that make them highly exposed to AI. Differences in exposure are, in turn, driven by the different task structures across establishments. In our empirical exercise, we will use the occupational mix of an establishment prior to the major advances in AI to infer its task structure and compute its exposure to AI. Formally, we assume that the set of tasks in the economy, $\mathcal{T}$, is partitioned into tasks performed by a set of distinct occupations and denote the set of tasks performed in occupation $o \in O$ by $\mathcal{T}^{o}$. Each establishment $e$ 's task structure is thus represented by the set of occupations that the establishment employs, denoted by $O_{e} \subset O$, and so $\mathcal{T}_{e}=\cup_{o \in O} \mathcal{T}^{o}$. For example, some establishments will employ accountants and their production will use the set of tasks accountants perform, while others

\footnotetext{
${ }^{8}$ Or that productivity gains, if present, have little effect on demand, potentially due to low passthrough rates.
} 
require the tasks performed by security analysts or retail clerks and thus hire workers into these occupations. In our empirical work, we will use the occupational indices provided by Felten, Raj and Seamans (2018, 2019), Webb (2020) and Brynjolfsson, Mitchell and Rock $(2018,2019)$ to identify the set of occupations involving tasks where AI can (or could) be deployed. We will then compute measures of AI exposure based on the occupational structure of an establishment. ${ }^{9}$

\section{DATA}

We next describe the BG data, document that it is broadly representative of employment and hiring trends across occupations and industries, present our AI exposure indices, and document their distribution across occupations and their evolution over time.

\subsection{Burning Glass Data}

Burning Glass (BG) collects data from roughly 40,000 company websites and online job boards, with no more than $5 \%$ of vacancies from any one source. BG applies a deduplication algorithm and converts the vacancies into a form amenable to data analysis. The coverage is the near-universe of online vacancies from 2010 onwards in the United States, with somewhat more limited coverage in 2007. Our primary sample comprises data from the start of 2010 until October 2018, though we also make use of the 2007 data. The vacancy data enumerate occupation, industry and region information, firm identifiers, and detailed information on occupations and skills required by vacancies, garnered from the text of job postings.

A key question concerns the representativeness of BG data given that the source of the vacancies is online job postings. Figure 1 shows that BG data closely track the evolution of overall vacancies in the US economy as recorded by the nationally representative Bureau of Labor Statistics (BLS) Job Openings and Labor Turnover Survey (JOLTS). The exception

${ }^{9}$ Using our notation, we can represent these indices as

$$
O \operatorname{Exp}^{o}=\frac{\int_{x \in \mathcal{T}_{\circ}^{\circ} \cap \mathcal{T}^{A}} \ell(x) d x}{\int_{x \in \mathcal{T}^{\circ}} \ell(x) d x}
$$

where $\ell(x)$ is average employment in task $x$ and we denote average employment in occupation $o$ by $\ell^{o}=$ $\int_{x \in \mathcal{T}_{o}} \ell(x) d x$. When $\ell(x)=\ell_{e}(x)$, which follows from our common technology assumption, the exposure to AI measure is equal to the employment weighted average of the occupation AI exposure measure:

$$
\frac{\sum_{o \in O_{e}} O E x p^{o} \ell^{o}}{\sum_{o \in O_{e}} \ell^{o}}=\frac{\sum_{o \in O_{e}} \frac{\int_{x \in \mathcal{T}^{o} \cap \mathcal{T}^{A}} \ell(x) d x}{\int_{x \in \mathcal{T}^{\circ}} \ell(x) d x} \ell^{o}}{\sum_{o \in O_{e}} \ell^{o}}=\frac{\int_{x \in \mathcal{T}_{e} \cap \mathcal{T}^{A}} \ell(x) d x}{\int_{x \in \mathcal{T}_{e}} \ell(x) d x}=\text { Exposure to } \mathrm{AI}_{e}
$$


is the downturn in BG postings data between 2015 and 2017. ${ }^{10}$ Appendix Figure A1 shows that over the 2010-2018 period, the occupational and industry composition in BG is closely aligned with both overall occupation employment shares from the OES and with industry vacancy shares from JOLTS. ${ }^{11}$

We make use of Burning Glass' detailed industry and establishment data. When this information is available from the text of postings, vacancies are assigned a firm name and a location, typically at the city level, as well as an industry code. We classify each firm as belonging to the industry in which it posts the most vacancies over our sample period. We define an establishment of a firm as the collection of vacancies pertaining to a firm and commuting zone. Commuting zones are groups of counties that, due to their strong commuting ties, approximate a local labor market (Tolbert and Sizer, 1996).

Of particular importance for our paper are BG's detailed skill and occupation coding. Vacancies in BG data contain information on skill requirements, scraped from the text of the vacancy. The skills are organized according to several thousand standardized fields. Groups of related skills are collected together into "skill clusters". Over 95\% of vacancies are assigned a six-digit (SOC) occupation code. ${ }^{12}$

We use these skill data to construct two measures of AI vacancies, narrow and broad. The narrow category includes a selection of skills relating to AI. ${ }^{13}$ The broad measure of AI includes skills belonging to the broader skill clusters of machine learning, artificial intelligence, natural language processing, and data science. A concern with our broad AI measure is that it may include various IT functions that are separate from core AI activities. For this reason, we focus on the narrow AI measure in the text and show the robustness of our main results with the broad occupation measure in the Appendix. Figure 2 shows the evolution

\footnotetext{
${ }^{10}$ While JOLTS measures a snapshot of open vacancies posted by establishments during the last business day of the month, BG counts new vacancies posted by the establishment during the entire month. We adjust the numbers of job openings in JOLTS to match BG's concept of vacancies, using the approach developed by Carnevale et al. (2014). The difference in concept between JOLTS and Burning Glass vacancies likely accounts for the downturn in BG postings data between 2015 and 2017.

${ }^{11}$ We note that BG data represent vacancy flows, while the OES reports employment stocks, and thus we do not expect the two data sources to align perfectly. Moreover, online vacancy postings tend to overrepresent technical and professional jobs relative to blue collar and personal service jobs (Carnevale et al., 2014).

${ }^{12}$ Six-digit occupation codes are highly granular, including occupations such as pest control worker, college professor in physics, and home health aide.

${ }^{13}$ The skills are Machine Learning, Computer Vision, Machine Vision, Deep Learning, Virtual Agents, Image Recognition, Natural Language Processing, Speech Recognition, Pattern Recognition, Object Recognition, Neural Networks, AI ChatBot, Supervised Learning, Text Mining, Support Vector Machines, Unsupervised Learning, Image Processing, Mahout, Recommender Systems, Support Vector Machines (SVM), Random Forests, Latent Semantic Analysis, Sentiment Analysis / Opinion Mining, Latent Dirichlet Allocation, Predictive Models, Kernel Methods, Keras, Gradient boosting, OpenCV, Xgboost, Libsvm, Word2Vec, Chatbot, Machine Translation and Sentiment Classification.
} 
of postings of narrow and broad AI vacancies in the BG data, highlighting the rapid takeoff of AI vacancies after 2015, as noted in the Introduction. While a sharp uptick is visible in all industries, the second panel of Figure 2 shows that the takeoff is particularly pronounced in the information, professional and business services, finance, and manufacturing sectors.

In what follows, our primary focus is on "AI-using sectors", and we drop establishments belonging to sectors that are likely to be producing AI-related products, namely the information sector (NAICS sector 51) and the professional and business services sector (NAICS sector 54). The former includes various information technology industries, likely to be selling AI products, while the latter contains industries such as management consultancy, likely to be integrating AI into other industries' production processes.

\subsection{AI Indices}

We study three measures of AI exposure. Each is assigned at the six-digit SOC occupation level, and each is designed to capture occupations concentrating in tasks that are compatible with the current capabilities of AI technologies.

The first measure is from Felten et al. (2019). It is based on data from the AI Progress Measurement project, from the Electronic Frontier Foundation. The Electronic Frontier data identify a set of nine application areas in which artificial intelligence has made progress since 2010, such as image recognition or language modeling. Felten et al. use Amazon MTurk to collect crowdsourced assessments of the relevance of each of these application areas to the 52 O*NET ability scales, e.g., depth perception, number facility, and written comprehension. The authors then construct the AI Occupational Impact for each O*NET occupation as the weighted sum of the 52 AI application-ability scores, where weights are equal to the O*NET-reported prevalence and importance of each ability in the occupation.

The second measure is from Webb (2020). Webb's analysis seeks to measure what tasks artificial intelligence can perform by identifying overlaps between claims about capabilities in AI patents and job descriptions in $\mathrm{O}^{*} \mathrm{NET}$. Occupations that have a larger fraction of such overlapping tasks are classified as more exposed.

The third measure is Suitability for Machine Learning (SML) from Brynjolfsson et al. (2019). To build this measure, Brynjolfsson et al. (2019) develop a 23-item rubric that enables the scoring of the suitability of any task for machine learning. They derive the SML scores by applying this rubric to the textual description of the full set of $\mathrm{O}^{*} \mathrm{NET}$ occupations using CrowdFlower, a crowdsourcing platform.

The three measures introduced above identify occupations that involve tasks in which AI 
algorithms have made (or could make) significant advances. The measures differ in the way they capture the applicability of AI to a task. Felten et al. and Webb focus on identifying tasks that fall within existing capabilities, either by relying on the reports from the AI Progress Measurement Project or based on the text of patents. The Brynjolfsson et al. SML index is more forward-looking, and identifies tasks that could be performed by machine learning/AI in the near term, even if outside the reach of existing capabilities. Given the short period of time covered by the BG data, we expect, and in fact find, that Felten et al.'s AI Occupational Impact and Webb's measure should have greater explanatory power for current adoption dynamics and establishment outcomes.

Figure 3 shows the distribution of our three indices by broad occupation categories and by one-digit industry. ${ }^{14}$ Figure 4 relates this same information to wages by plotting average AI exposure by occupational wage percentile for each index. The figures confirm that these three measure capture different aspects of AI. The Felten et al. measure, for example, is particularly high for managers, professionals and office and administrative staff and is very low for service, production and construction workers, capturing the fact that these occupations involve various manual tasks that cannot currently be performed by algorithms. The Webb measure is not particularly high in sales occupations and shows a strong positive relationship with occupational wage percentiles. In contrast, the SML measure is high for office and administrative occupations, and for sales occupations, and (perhaps surprisingly) above average for personal services, but is low for professional occupations and most bluecollar and service occupations. Consequently, SML has no systematic relationship with occupational wage percentiles. ${ }^{15}$

\section{EMPIRICAL STRATEGY}

Our empirical strategy links measures of AI activity and job posting outcomes to AI exposure, where both outcome and exposure variables are measured at the establishment level.

We estimate the following regression model:

$$
\Delta y_{e, t-t_{0}}=\beta A I_{e, t_{0}}+\mathbf{x}_{e, t_{0}}^{\prime} \gamma+\varepsilon_{e, t-t_{0}}
$$

\footnotetext{
${ }^{14}$ The broad occupational categories are those utilized by Autor (2019) and aggregate six-digit occupations into 12 roughly one-digit categories.

${ }^{15}$ Another notable difference is that the Webb index finds very little AI-suitability in either office or sales occupations. Alongside his AI index, Webb (2020) creates a separate software exposure index, pertaining to traditional non-AI software, that detects substantial software-suitability in office, administrative, and sales occupations. We use this index as a control in our robustness checks.
} 
where $e$ denotes establishment, $\Delta y_{e, t-t_{0}}$ denotes the change in one of our establishment-level outcomes between 2010-12 and 2016-18, $A I_{e, t_{0}}=\sum_{o}$ share postings $_{o e, t_{0}} \times$ AI score $_{o}$ is one of our three measures of establishment AI exposure calculated using establishment data for 2010 through 2012, and $\mathbf{x}_{e, t_{0}}$ is a vector of baseline controls, including industry dummies, firm size decile dummies, a dummy for the commuting zone (CZ) in which the establishment is located and, in some specifications, a set of firm fixed effects. ${ }^{16}$ Finally, $\varepsilon_{e, t-t_{0}}$ is an error term representing all omitted factors.

Our primary interest is in the coefficient $\beta$, which captures the relationship between AI exposure and the outcome variable. We standardize the establishment AI exposure measure by dividing it by its weighted standard deviation, with weights given by vacancies in 20102012. Hence, $\beta$ is the change in the outcome variable associated with a standard deviation difference in AI exposure.

The three main outcome measures we focus on for $\Delta y_{e, t-t_{0}}$ are AI vacancies, changes in job skill requirements of posted vacancies, and overall non-AI hiring, all measured at the establishment level.

\section{AI Exposure and AI Vacancies}

We first document that AI exposure predicts establishment-level AI activity, as proxied by our measure of narrow AI vacancies. Table 1 presents the main estimates. Panel A of this table shows the relationship between AI exposure based on Felten et al.'s index and growth in AI vacancies, while the subsequent two panels present results for our other measures of AI exposure. We estimate regression models based on (6), with the left-hand side variable defined as the change in the inverse hyperbolic sine of AI vacancies between 2010-12 and 2016-18. ${ }^{17}$ We focus on weighted specifications, using baseline establishment vacancies as weights. In the text, we report heteroscedasticity-robust standard errors that allow for arbitrary cross-sectional correlation across the establishments of each firm, and consider alternative standard errors in the Appendix.

Column 1 is our most parsimonious specification and includes no covariates, thus depicting the unconditional bivariate relationship. The coefficient estimate in Panel A of

\footnotetext{
${ }^{16}$ We pool 2010-12 data and, separately, 2016-18 data to improve precision.
}

${ }^{17}$ The inverse hyperbolic sine transformation is given by:

$$
\ln \left(x+\sqrt{\left(x^{2}+1\right)}\right) .
$$

For small values of $x$, this approximates a proportional change, but is well defined when $x=0$, which is a frequent occurrence in our sample of establishments. 
$\beta=15.96$ is precisely estimated $(\mathrm{SE}=1.73)$ and shows a sizable association between $\mathrm{AI}$ exposure and AI vacancies. This estimate implies that a one standard deviation increase in AI exposure - which corresponds to the difference between finance and mining and all extraction - is associated with approximately a $16 \%$ increase in AI vacancies.

The remaining columns explore the robustness of this relationship. Column 2 controls for firm size decile and commuting zone fixed effects. The coefficient estimate of AI exposure declines slightly to 13.82, but is now more precisely estimated. Column 3 additionally adds three-digit (NAICS) industry fixed effects. Reflecting the sizable variation in AI exposure across industries shown in Figure 3 above, these controls are more important for our regressions, and they reduce the magnitude of our estimate by about a third, to 9.19, but the standard error of the estimate also declines (to 1.21).

Column 4 goes one step further and includes a full set of firm fixed effects, so that now the comparison is among establishments of the same firm that differ in their AI exposure. The estimate of $\beta$ is similar to the bivariate relationship reported in column 1, 16.53, albeit slightly less precise, since all of the cross-firm variation is now purged.

Figure 3 documented significant differences in AI exposure across occupations. This raises the concern that our results may be confounded by secular trends across broad occupational categories. Columns 5 and 6 additionally control for the baseline shares of vacancies that are in sales and administration, two of the broad occupational categories that have been in decline for other reasons (e.g., Autor and Dorn, 2013). These controls do not substantially change the estimate in either column, which remain, respectively, at $9.75(\mathrm{se}=1.20)$ and $16.87(\mathrm{se}=1.86){ }^{18}$

The first panel of Figure 5 shows the specification from column 3 in the form of a bin scatterplot, where each bin represents about 50,000 establishments. The relationship between AI exposure and AI vacancy postings is fairly close to linear across the distribution and does not appear to be driven by outliers. The first panel of Figure 6 provides a complementary visualization, depicting the evolution of AI vacancies for the four quartiles of the establishment AI exposure measure. It shows that the top two quartiles post significantly more AI vacancies and drive the surge in AI vacancies after 2015. ${ }^{19}$

\footnotetext{
${ }^{18}$ Table A1 in the Appendix shows that the results in Panel A are also robust if we include the baseline shares of ten broad occupational categories. For example, the coefficients in the specifications that parallel columns 5 and 6 are, respectively, $7.24(\mathrm{se}=1.44)$ and $13.70(\mathrm{se}=2.12)$. However, some of the results for the Webb and SML AI exposure measures are sensitive to these controls.

${ }^{19}$ Our exposure measure is a "shift-share" instrument, and the heteroscedasticity-robust standard errors are not the most conservative ones because they do not recognize the additional correlation coming from the covariation of these shares (Adao, Kolesar \& Morales, 2019; Borusyak, Hull \& Jaravel, 2021). Appendix Table A2 repeats Table 1 with standard errors from Borusyak et al (2021), with similar results.
} 
Our simple measure of exposure to AI explains a significant fraction of the increase in AI vacancy posting. To document this point, we calculate the adjusted $R^{2}$ associated with the specifications in Table 1. For comparison, in Appendix Table A3 we also compute the share of the increase in AI activity associated with initial occupation composition by estimating a version of our main regression equation (6), with the share of establishment vacancies in each detailed occupation in 2010-2012 as regressors. The adjusted $R^{2}$ of the Felten et al measure of AI exposure is 0.0256 . The adjusted $R^{2}$ when initial occupation shares are used as regressors is $0.10 .{ }^{20}$ Hence, our AI exposure measure accounts for more than one-quarter of the AI adoption associated with baseline occupation structures.

Panels $\mathrm{B}$ and $\mathrm{C}$ of Table 1 repeat the Panel A regressions using the Webb and SML indices of AI exposure. Estimates using the Webb measure, reported in Panel B, are similar to those in Panel A in the first four specifications, though they are not fully robust to controls for the baseline shares of sales and administration vacancies in columns 5 and 6 . The second panel of Figure 5 shows that the bin scatter plot with the Webb measure looks similar tothough noisier than - the one in the first panel with the Felten et al. measure, and Figure 6 confirms that the surge in AI vacancies is again driven by the top two quartiles. Given that the Felten et al. and Webb indices capture different components of AI exposure (recall Figure 3), the broadly similar picture they depict is reassuring. However, from Appendix Table A3, the partial $R^{2}$ associated with the Webb measure is 0.0074 , roughly one quarter of the corresponding $R^{2}$ for the Felten et al measure.

Results with the SML index in Panel C are broadly similar, but significantly weaker. There is a positive association between the SML-based measure of AI exposure and AI vacancy growth without any covariates, but when three-digit industry fixed effects are included, this relationship becomes negative. The proximate explanation for this pattern is that the sales and administration occupations have a high SML score, as noted above, and are negatively associated with AI adoption. When we control for the baseline shares of these occupations in columns 5 and 6, the positive relationship in column 1 is restored. The third panels of Figures 5 and 6 show a less clear pattern relative to the Felten et al. and Webb measures as well. The bin scatterplot confirms the lack of a robust relationship between exposure to AI based on the SML measure and AI vacancies (from the specification in column 3), and the evolution of AI vacancy growth by exposure quartiles in Figure 6 no longer shows a monotone pattern. These weaker results with SML motivate our greater emphasis on the

\footnotetext{
${ }^{20}$ We estimate the regressions in Appendix Table A3 on a $10 \%$ sample, since there are many regressors in the model with initial occupation shares. We have verified that the adjusted $R^{2}$ is similar in the sample and the full data when the regressors are our measures of AI exposure.
} 
results using the Felten et al. and Webb measures in the remainder of the paper.

A concern with the estimates in Table 1 is that the AI measures may be proxying for exposure to non-AI digital technologies. If so, this would cloud the interpretation of our estimates as primarily capturing the impacts of AI exposure on establishment outcomes. We check for this possibility in Table 2 by additionally controlling for Webb's measure of exposure to "software", which is calculated analogously to his AI exposure measure, but focusing on occupations and tasks suitable for traditional software and digital technologies. The inclusion of the software exposure measure has little impact on the coefficients of interest, particularly in the case of the Felten et al. index. For example, in the most loaded specification (column 6), the point estimate is now 17.47 with a standard error of 1.90, as compared to 16.87 and a standard error of 1.86 in Table 1. The software exposure measure itself does not have a consistent association with AI vacancy growth: it is positive and statistically significant in some specifications, small and insignificant in others, and negative and significant in yet others. This set of estimates bolsters our confidence that the AI exposure variable identifies meaningful variation in the suitability of establishment task structure for AI, and that this variation is distinct from exposure to traditional software and digital technologies.

We provide several robustness checks on these basic patterns in the Appendix. In Table A4, we report estimates for AI vacancy growth using AI exposure calculated using establishments' occupational structures in 2007 rather than 2010. Although this greatly reduces the sample size as many establishments operating in both 2010 and 2018 are not present in the 2007 data, we obtain qualitatively similar results to those in Table 1.

In Table A5, we replace the narrow AI vacancy measures used in Table 1 with the broad AI vacancy indices discussed above (see Figure 2), and in Table A6, we use the change in the share of AI vacancies among all vacancy postings as the dependent variable. The results in both tables corroborate our main findings in Table 1 and, if anything, are stronger and more stable, especially with the Webb measure. The quantitative magnitudes implied by the estimates in these tables are comparable to our baseline estimates. For example, with the Felten et al. measure and the specification in column 6, an additional standard deviation of AI exposure is associated with a 19 percentage point increase $(\mathrm{se}=0.02)$ in the share of AI vacancies between 2010 and 2018.

We also explored firm-level variants of the establishment-level models above. Because of the many zeros in the data, the establishment-level estimates do not aggregate cleanly to firm-level estimates. As shown in columns 1 and 2 of Table A7, these estimates are generally imprecise and inconsistently signed. However, when we estimate models for the firm-level mean of establishment AI vacancy growth (columns 3 and 4) or models with share of AI 
vacancies (columns 5 and 6), the estimates are very similar to our main results in Table 1.

In summary, the data point to a recent surge in AI-related hiring, and our regression evidence reveals that establishments whose task structures enable the use of artificial intelligence technologies have substantially increased their AI-related postings. This evidence suggests that an important component of AI activity is linked to the types of tasks performed in an establishment - though it does not preclude the possibility that AI activity has other drivers, such as the development of new products or business models.

\section{AI AND NEW SKILLS}

Having established the link between AI suitability and AI activity/hiring at the level of establishments, we now turn to the broader labor market implications of growing AI adoption. AI is intended to supplement, replicate, and in some cases, exceed human-level intelligence in a variety of tasks. We therefore anticipate that establishments with task structures that are suitable for AI will tend to change the types of worker skills they demand.

To investigate whether AI exposure predicts skill demands in non-AI jobs, we build on work by Deming and Noray (2020) who document such changes associated with broader IT-related activity. We adapt their measure of change in skill demands to establishments and separate their gross skill change measure into negative and positive changes, capturing the disappearance of existing skills and the emergence of new skills:

$$
\begin{gathered}
\text { negative skill change } e_{e, t_{2}, t_{1}}=-\min \left\{\sum_{s=1}^{S}\left[\left(\frac{\text { skill }_{e, t_{2}}^{s}}{\operatorname{vacancies}_{e, t_{2}}}\right)-\left(\frac{\text { skill }_{e, t_{1}}^{s}}{\text { vacancies }_{e, t_{1}}}\right)\right], 0\right\}, \text { and } \\
\text { positive skill change }_{e, t_{2}, t_{1}}=\max \left\{\sum_{s=1}^{S}\left[\left(\frac{\text { skill }_{e, t_{2}}^{s}}{\text { vacancies }_{e, t_{2}}}\right)-\left(\frac{\text { skill }_{e, t_{1}}^{s}}{\text { vacancies }_{e, t_{1}}}\right)\right], 0\right\},
\end{gathered}
$$

Here, skill $e_{e, t}^{s}$ is the number of times skill $s$ is posted by establishment $e$ in year $t$, which we normalize by dividing it by the total number of vacancies posted by that establishment. The negative skill change measure therefore represents a decline in the frequency with which some of the skills that were formerly posted appear in vacancies, while the positive skill change measure captures increases in the frequency with which other skills are posted in vacancies - which may include the addition of skills that were not previously posted. We calculate these measures for non-AI vacancies and, as before, for all establishments except those in the professional and business services and information technology sectors (51 and $54)$. 
Tables 3 and 4 show that establishment AI exposure is robustly associated with negative and positive skill changes. For example, in the first panel of Table 3, which focuses on negative skill changes, column 1 shows an estimate of 0.83 , which indicates that a one standard deviation in the Felten et al. exposure measure is associated with a 0.83 absolute decline in the per-vacancy frequency with which skills previously demanded are posted $(\mathrm{se}=$ 0.09). This is a large change compared to the mean negative skill change in our sample, 4.70 , and suggests that the deployment of AI technologies goes hand-in-hand with significant skill redundancies. Equally interesting is the pattern in Table 4, which shows that AI exposure is associated with demand for new skills. Column 1 of this table shows that a one standard deviation increase in the Felten et al. AI exposure measure is associated with 0.95 absolute increase $(\mathrm{se}=0.08)$ in the per-vacancy frequency of skills that were either demanded less frequently previously or were not previously demanded. This too is a sizable impact as compared to the mean positive skill change in our sample of 6.30 .

These patterns are quite robust, as is shown in the remaining columns and panels of Tables 3 and 4. Each of the three AI exposure measures - Felten et al., Webb, and SMLpredict both negative and positive establishment-level skill changes between 2010 and 2018 . Paralleling our findings at many points in the paper, the Felten et al. measure proves to have the most stable and largest quantitative relationship to the outcome variable, followed by Webb and SML. In particular, all measures prove robust to the inclusion of firm size deciles, commuting zone dummies, and controls for initial establishment vacancy structures in sales and administrative occupations. All three are also robust to the inclusion of firm fixed effects when we look at negative skill changes. The association between AI exposure and positive skill changes is no longer present when we include firm fixed effects, however, suggesting that the addition of new skills may not be localized to highly-exposed establishments, but rather occur throughout the firm or at the headquarters. Finally, Appendix Tables A8 and A9 additionally confirm that controlling for Webb's measure of exposure to software, as we did in Table 2, has little effect on the relationship between AI exposure and changes in skill demands. Akin to the Table 2 results, this pattern underscores that the predictive relationship between AI exposure and establishment outcomes is distinct from that for exposure to traditional software.

To provide insight into what types of skills are affected by AI, we estimate the same models as in Tables 3 and 4 within 28 skill families created by Burning Glass. These families, which are enumerated in Figures 7 and 8, cover major job activities and skill sets within white-collar, blue-collar, and service occupations. We find that both positive and negative skill changes concentrate in the same, relatively small subset of skills. This is shown in 
Figure 7, which reports point estimates and 95\% confidence intervals for a regression of establishment negative skill change separately for each skill family on establishment AI exposure using each of our three measures. Using both the Felten et al and Webb measures, AI exposure predicts increasing demands for skills relating to engineering, analysis, marketing, finance, and information technology. Conversely, Figure 8 presents estimates for the relationship between AI exposure and positive skill change by skill family. For the Felten et al and Webb measures, AI-exposed establishments have lower demands for skills in the same families in which negative skill change is greatest.

The finding that AI exposure is associated with significant changes in the skills listed in vacancies bolsters our confidence that AI adoption has real effects on the task content of non-AI jobs - enabling firms to replace some of the tasks previously performed by workers, making certain skills redundant, while simultaneously generating demand for new skills. These results are also consonant with our theoretical model in Section 2, which suggests that AI adoption will induce churn of tasks performed by workers as some tasks previously performed by humans are taken over by algorithms.

\section{AI AND JOBS}

Our theory leaves open the possibility that AI may increase or reduce overall (and non-AI) hiring. We next investigate the effects of AI exposure on vacancies for non-AI positions.

\subsection{AI Exposure and Establishment Hiring}

Table 5 turns to the relationship between AI exposure and hiring. The structure of the table is identical to that of Table 1 except that the left-hand side variable is now the change in the inverse hyperbolic sine of total non-AI vacancies (and there are two extra columns, which we describe below). The non-AI vacancy measure is chosen so as to focus on the effects of AI activity on establishment hiring exclusive of already-reported impact on AI hiring itself. As before, we drop professional and business services and information technology sectors (NAICS 51 and 54).

In Panel A, where we focus on Felten et al.'s measure, we see a robust negative association between AI exposure and subsequent non-AI hiring. The estimate in column 1 is -13.80 (se $=4.22$ ), indicating that a one standard deviation increase in AI exposure is associated with a roughly $14 \%$ decline in overall non-AI vacancies. (We interpret the economic magnitudes of these point estimates below.) This coefficient estimate remains stable when we control for 
firm size decile, commuting zone and three-digit industry fixed effects in columns 2 and 3.

In column 4, we replace firm-level covariates with firm fixed effects while retaining the commuting zone dummies from the prior column. This is a stringent specification since we are now comparing across establishments of the same firm that differ in their AI exposure. In this specification, the point estimate for AI exposure is -4.81 , which is about half of the magnitude in the preceding column. Simultaneously, the estimates become more precise as the standard error falls from 4.08 to 1.44. The relationship between AI exposure and non-AI vacancies remains comparable when we include the baseline shares of sales and administration occupations in columns 5 and 6: $-12.42(\mathrm{se}=4.01)$ and -4.04 ( $\mathrm{SE}=1.47)$, respectively. ${ }^{21}$

We also investigated whether these estimates are driven by establishments that posted jobs in 2010-2012 and then stopped posting in 2016-2018 (which may reflect either true zero vacancy postings or establishment exits, perhaps for sampling reasons). Columns 7 and 8 limit the sample to establishments that posted in 2016-2018. The estimates are now somewhat smaller, but still negative and statistically significant at 5\%: -8.38 (se $=3.46)$ in column 7, with three-digit industry fixed effects, and $-3.56(\mathrm{SE}=1.86)$ in column 8 , with firm fixed effects. ${ }^{22}$

How large are the effects reported in Panel A? The interpretation of the regression coefficients is not straightforward because our outcome variable is vacancy flows, which differs from the stock of employment. To estimate the implied impact on employment, we cumulate vacancies between 2010 and 2018 to create a measure of 2018 employment for each establishment. Then we regress our measure of cumulative hiring between 2010 and 2018 on AI exposure in 2010 exactly as in Table 5. ${ }^{23}$ Table A11 in the Appendix reports the results of this exercise. In Panel A, with Felten et al.'s measure of AI exposure, the regression coefficient in column 1 of -7.24 ( $\mathrm{se}=4.66)$ implies that a one standard deviation in AI exposure is associated with a 7.2\% decline in non-AI employment between 2010 and 2018. Since a one standard deviation increase in AI exposure is quite large, this is a sizable but not implausible relationship. We also note that because this coefficient estimates the relative change in

\footnotetext{
${ }^{21}$ Since AI exposure predicts an increase in AI vacancies, it is not self-evident whether the implied impact on total vacancies (inclusive of AI hiring) is also negative. We show in Appendix Table A10 that the answer is yes, as expected, since AI vacancies are a tiny share of total vacancies.

${ }_{22}$ In Appendix Table A12 we calculate the standard errors from Borusyak et al (2012) for the specifications in Table 5 to account for the shift-share structure of our AI exposure measure. The standard errors change little.

${ }^{23}$ More specifically, we assume that establishment employment, $\ell_{e, t}$, follows the law of motion $\ell_{e, t+1}=$ $f v_{e, t}+(1-s) \ell_{e, t}$, where $v_{e, t}$ denotes the establishment's vacancies, $f$ is the vacancy fill rate and $s$ is the separation rate. We calculate employment in 2010 by assuming that the establishment is in steady state initially, and we compute employment in 2018 by iteratively solving the law of motion forward. We set $s=0.4$ to match the 2018 annual separation rate from JOLTS. We thank Andreas Mueller for suggesting this exercise.
} 
non-AI hiring at more versus less AI-exposed establishments, it does not imply an aggregate reduction in total hiring. ${ }^{24}$

Panel B of Table 5 turns to Webb's measure of AI exposure. The pattern is broadly similar to the one we see in Panel A but less stable. The coefficient estimate without any covariates in column 1 is -17.24 ( $\mathrm{se}=3.72$ ). It is comparable in column 2 when we control for $\mathrm{CZ}$ and three-digit industry fixed effects. However, the estimate declines substantially to -2.22 ( $\mathrm{se}=0.93)$ in column 4 when we control for firm fixed effects, and is inconsistent in sign and magnitude in columns 5 through 8. Finally, when we use SML in Panel C, there is no consistent evidence for a negative association between AI exposure and non-AI vacancy postings (negative and statistically significant in column 6 , but positive in six of eight columns and significantly so in two cases).

As in Table 2, we next control for Webb's measure of exposure to software in order to distinguish the effects of other (traditional) software applications from AI. The results reported in Table 6 document that the software exposure measure itself has no consistent association with non-AI hiring, while the effects of AI exposure remain very similar to our baseline estimates in Table 5. For example, the estimate using Felten et al.'s measure in column 1 is -14.62 , compared to -13.80 for the same specification in Table $5 .^{25}$

We showed in Figure 2 that AI adoption sharply accelerated around 2015 after having grown comparatively slowly in the prior five years. This discontinuous growth provides an opportunity to test whether any potential association between AI exposure and non-AI hiring fits this timing. We perform this exercise in Table 7, where we break the outcome period

\footnotetext{
${ }^{24}$ One can combine the reduced-form estimates in Tables 1 and 5 to obtain a Wald estimate of how AI activity driven by differences in tasks structures across establishments affects non-AI hiring. For example, the estimates in column 4 of Tables 1 and 5, using Felten et al.'s measure, yield an elasticity of - 0.3 - that is, a $10 \%$ increase in AI adoption is associated with a $10 \% \times 4.81 / 16.53=3 \%$ decline in non-AI hiring. Between 2010 and 2018, the increase in AI vacancies ranged from $218 \log$ points in finance to $198 \log$ points in manufacturing. Assuming that $1.6 \%$ of these increases - the partial $R^{2}$ of our AI exposure measure in Table 1 - were driven by task-level substitution of algorithms for labor, one may infer that this type of AI adoption led to a decline of $1 \%$ in non-AI hiring in finance and $0.92 \%$ in manufacturing. These estimates should be interpreted with caution, since they ignore general equilibrium effects and spillovers, and the partial $R^{2}$ may over or understate the role of task substitution in AI activity. Indeed, the OLS relationship between AI adoption and hiring, reported in Appendix Table A13, is positive. This underscores that other sources of variation, including possible links between an establishment's growth potential and its AI activity, matter more for AI adoption than the baseline task structure captured by our AI exposure measure.

${ }^{25}$ Another prediction of our conceptual framework is that hiring should decline particularly in occupations that are themselves highly exposed to AI. Consistent with this prediction, all of our three AI exposure measures predict a decline in "at-risk" vacancies. We are nevertheless cautious in interpreting these specifications and do not report them because they suffer from potential mean reversion. In particular, because the exposure measure is equal to the share of establishment postings that are at-risk in 2010-2012, any mean reversion in this measure will induce a spurious negative relationship between an establishment's at-risk vacancy share in 2010-2012 and its subsequent change.
} 
of 2010-2018 into two subperiods, 2010-2014 and 2014-2018, and estimate a subset of the specifications in Table 5 for these sub-intervals.

Focusing on Felten et al.'s AI exposure measure in Panel A, we see no economically or statistically significant relationship between AI exposure in 2010 and non-AI hiring during the years 2010-14, while there is a strong negative association for 2014-18, during the period of rapid AI takeoff. In the baseline regression for 2014-18 (column 5), the point estimate is -11.94 ( $\mathrm{se}=3.80$ ), indicating that a one standard deviation increase in AI exposure is associated with an approximately $12 \%$ decline in overall non-AI vacancies. This estimate remains stable and becomes more precise when we control for firm size deciles, commuting zone controls, and three-digit industry fixed effects in column 6. The relationship also remains comparable when we include the baseline shares of sales and administration occupations in column 7 -coefficient of -10.60 with a standard error of 2.82 . This result is robust to firm fixed effects, added in column 8, though, as before, their inclusions reduces the magnitude of the relationship. Panels $\mathrm{B}$ and $\mathrm{C}$ report the same specifications for the Webb and SML measures, respectively. As in Table 5, the relationships between non-AI hiring and AI exposure are less consistent and robust for these indexes.

Table A14 in the Appendix presents results at the firm level, which are, on the whole, similar to the establishment-level results. Table A1, on the other han5d, depicts similar, even if slightly smaller, estimates with average establishment size weights (rather than baseline establishment size weights as in Table 5).

In sum, the evidence using the Felten et al. and Webb measures of AI exposure shows statistically significant and economically meaningful negative effects, especially between 2015 and 2018, the period during which AI activity surged.

\subsection{AI and Industry Employment}

Associated with the surge in AI activity, there may also be industry-level changes that potentially offset or amplify the establishment-level consequences. To investigate whether more exposed industries are contracting (or expanding), we aggregate our AI exposure measure to the CZ-by-industry level and merge it with employment data. We proxy industry-level AI activity using the mean occupation AI exposure across the six-digit occupations posted in each sector by CZ cell during 2010-12, weighted by the number of vacancies posted in each occupation. We measure the change in (the log of) industry-by-CZ employment using County Business Patterns data (CBP) for 2000-2016. Because of increased suppression of industry-by-location data in the CBP starting in 2017, our analysis of CBP data ends in 
2016, thus (unfortunately) excluding the last several years of rapid AI expansion. ${ }^{26}$

The results are reported in the first three columns of Table 8, which again contins one panel for each AI exposure measure. The outcome variable in these regressions is industryby-CZ employment. All models include industry fixed effects, commuting zone fixed effects, and baseline occupational shares in sales and administration. The standard errors are robust to heteroscedasticity and correlation within commuting zones.

Columns 1 and 2 examine trends in industry employment during 2003-2007 and 20072010, before the major AI advances that followed. These columns show that industry AI exposure in 2010 does not predict differential employment behavior before 2010; thus, threedigit industries with different AI exposure were on roughly parallel trends before the pickup in AI activity in the late 2010s. This pattern is essentially unchanged after 2010. We do not see consistent positive or negative effects associated with AI exposure between 2010 and 2016. For example, the estimate in column 3 is -0.05 ( $\mathrm{se}=0.08)$. The point estimate implies very small effects associated with industry AI exposure: a one standard deviation increase in industry AI predicts an economically small and statistically insignificant $0.049 \%$ decline in industry employment. Panels B and $\mathrm{C}$ of the table show similar results using the Webb and SML measures in place of the Felten et al. index.

This set of null results may indicate that it is premature to detect AI's impact on industry reorganization or growth. Indeed, our calculations in footnote 24 suggest that the present effects of AI adoption on even some highly-impacted sectors, such as finance, might still be small. These results might also indicate that much of the effect of AI on employment, if eventually present, will occur within industries.

\subsection{Employment and Wages in AI-Exposed Occupations}

As a second approach to measuring impacts that extend beyond firms, columns 4 through 9 of Table 8 assess whether occupations with greater AI exposure exhibit differential employment or wage trends after the onset of rapid AI hiring. For this analysis, we use occupational employment and wage information from the US BLS Occupational Employment Statistics (OES) data. This establishment-based data series provides more accurate estimates of employment and wages in occupations than is available from household surveys.

The observations in this table are at the six-digit occupation level, and the dependent variable is the sum of employment in a six-digit occupation across all industries (excluding sectors 51 and 54). In all columns, we control for three-digit occupation fixed effects and use

\footnotetext{
${ }^{26}$ In processing the CBP data, we use the harmonization and imputation procedures developed by Fabian Eckert, Teresa Fort, Peter Schott, and Natalie Yang, available at http://fpeckert.me/cbp/.
} 
baseline employment as weights. The standard errors are robust against heteroscedasticity. In columns 4 through 6 , the dependent variable is change in employment, while in columns 7 though 9 it is change in the (log) average wage in the occupation.

The results for employment and wage growth using each of the AI exposure measures are similar to the industry-level findings in earlier columns: we detect no differential employment or wage behavior in more AI-exposed occupations after $2010 .^{27}$

Our evidence is fairly clear that there is no systematic aggregate relationship between AI exposure and industry and occupation-level outcomes. Our overall interpretation is that while AI technologies appear to be changing task and skill composition at exposed establishments and firms, any aggregate effects of AI are too small to detect. ${ }^{28}$

\section{Conclusion}

There is much excitement and quite a bit of apprehension about AI and its labor market effects. In this paper, we explored the nature of AI activity in the US labor market and its consequences for skill change, hiring, and industry and occupation level changes in employment and earnings. We have three main findings:

1. We see a surge in AI activity, particularly after 2015, proxied by vacancies seeking workers with AI skills, and this surge is driven by establishments with high exposure to AI-meaning that their task structure in 2010 was suitable for the AI technologies that are subsequently introduced. This pattern is highly robust with two of our three AI-exposure measures - those based on the indices constructed by Felten et al. and Webb-and still present but less robust with our third measure, SML, based on Brynjolfsson, Mitchell and Rock's work.

2. We estimate consistent and robust changes in the skills demanded by high-exposure establishments. In particular, establishments with task structures suitable for AI cease to post vacancies that list a range of previously-sought skills and start posting additional skill requirements. This evidence suggests that some of the tasks that workers

\footnotetext{
${ }^{27}$ Differently from our industry results, we detect a significantly faster increase in the employment of more exposed occupations between 2007 and 2010 when using the Felten et al. AI measure. This may reflect fast expansion in some IT-related occupations that have high Felten et al. scores, or it may be a chance finding given the large number of point estimates reported in this table.

${ }^{28}$ One alternative reading of these results is that $\mathrm{AI}$ is displacing and reinstating tasks at approximately the same rate, yielding no net effect on labor demand. Our main results do not support this interpretation, however, since we find significant declines in non-AI vacancies at exposed establishments.
} 
used to perform in these establishments are no longer required, while new skills are simultaneously being introduced.

3. With two of our three measures, we find that AI-exposed establishments reduce their non-AI and overall hiring. These results are statistically significant, economically sizable, and robust with the Felten et al. measure, and robust in most specifications with the Webb measure. We do not detect such negative employment effects with SML, which is as expected, since the relationship between AI exposure and AI hiring is much less robust and stable with SML as well.

In contrast to these three findings, we do not detect any relationship between AI exposure and employment or wages at the occupation or industry level.

The totality of the results reported above on the labor market effects of AI convince us that the AI is having real effects on establishments that are exposed to this new technology: there is a significant surge in vacancies for AI workers in establishments with task structures that are more suitable for AI; skill churn increases differentially at AI-exposed establishments, with both greater retirement of previously-posted skills and greater introduction of new skills; and finally, AI-exposed establishments appear to be reducing their non-AI hiring. These patterns are consistent with the hypothesis that AI-powered algorithms are being used for substituting for human skills. However, while AI technologies appear to be changing task and skill composition at exposed establishments, any aggregate effects of AI, if present, are not yet detectable - plausibly because AI technologies are still in their infancy and have spread only to a limited part of the US economy.

Our results leave open important questions and have evident shortcomings. First, it will be valuable to further explore and understand the juxtaposition of negative establishmentlevel impacts and zero aggregate effects. Second, our focus on AI adoption driven by the task structure of establishments may exclude other types of AI impacts that are less related to task structures, such as the use of AI to launch new products and services. These applications could have different and possibly more positive effects on jobs. Naturally, our estimates are not informative about AI applications that are missed by our AI exposure measures. Finally, because the next generation of AI-enabled technologies will likely have different capabilities from the current generation, our results do not foretell whether future AI technologies will prove more complementary or more substitutable with human capabilities. 


\section{REFERENCES}

Acemoglu, Daron, and David Autor. 2011. Skills, tasks and technologies: Implications for employment and earnings. Handbook of Labor Economics 4:1043-1171.

Acemoglu, Daron, and Pascual Restrepo. 2018. The race between man and machine: Implications of technology for growth, factor shares, and employment. American Economic Review 108(6):1488-1542.

Acemoglu, Daron, and Pascual Restrepo. 2019a. Automation and new tasks: How technology displaces and reinstates labor. Journal of Economic Perspectives 33(2):3-30.

Acemoglu, Daron, and Pascual Restrepo. 2019b. The wrong kind of AI? Artificial intelligence and the future of labour demand. Cambridge Journal of Regions, Economy and Society 13(1):25-35.

Acemoglu, Daron, and Pascual Restrepo. 2020. Robots and jobs: Evidence from US labor markets. Journal of Political Economy 128(6):2188-2244.

Acemoglu, Daron, Claire Lelarge, and Pascual Restrepo. 2020. Competing with robots: Firm-level evidence from France. AEA Papers and Proceedings 110:383-388.

Adão, Rodrigo, Michal Kolesár, and Eduardo Morales. 2019. Shift-share designs: Theory and inference. The Quarterly Journal of Economics 134(4):1949-2010.

Alekseeva, Liudmila, José Azar, Mireia Giné, Sampsa Samila, and Bledi Taska. 2021. The demand for AI skills in the labor market. Labour Economics 71.

Autor, David, Lawrence Katz, and Alan Krueger. 1998. Computing inequality: Have computers changed the labor market? The Quarterly Journal of Economics 113(4):1169-1213.

Autor, David, Frank Levy, and Richard Murnane. 2003. The skill content of recent technological change: An empirical exploration. The Quarterly Journal of Economics 118(4):1279-1333.

Autor, David, and David Dorn. 2013. The growth of low-skill service jobs and the polarization of the US labor market. American Economic Review 103(5):1553-1597.

Autor, David. 2019. Work of the past, work of the future. AEA Papers and Proceedings 109:1-32.

Agarwal, Ajay, Joshua S. Gans, and Avi Goldfarb. 2018. Prediction machines: The simple economics of artificial intelligence. Cambridge: Harvard Business Review Press.

Azar, José, Ioana Marinescu, Marshall Steinbaum, and Bledi Taska. 2020. Concentration in US labor markets: Evidence from online vacancy data. Labour Economics, 66. 
Babina, Tania, Anastassia Fedyk, Alex Xi He, and James Hodson. 2020. Artificial intelligence, firm growth, and industry concentration. Unpublished manuscript, Columbia University.

Bessen, James E., Stephen Michael Impink, Lydia Reichensperger, and Robert Seamans. 2018. The business of AI startups. Unpublished manuscript, Boston University, School of Law.

Bessen, James, Maarten Goos, Anna Salomons, and Wiljan Van der Berge. 2019. Automatic reaction: What happens to workers at firms that automate? Unpublished manuscript, Boston University, School of Law.

Bonfiglioli, Alessandra, Rosario Crino, Harald Fadinger, and Gino Gancia. 2019. Robot imports and firm level outcomes. Unpublished manuscript. Queen Mary University of London.

Borusyak, Kirill, Peter Hull, and Xavier Jaravel. 2021. Quasi-experimental shift-share research designs. Review of Economic Studies forthcoming.

Bresnahan, Timothy. 2019. Artificial intelligence technologies and aggregate growth prospects. Unpublished manuscript, Stanford.

Brynjolfsson, Erik, Tom Mitchell, and Daniel Rock. 2018. What can machines learn, and what does it mean for occupations and the economy? AEA Papers and Proceedings 108:43-47.

Brynjolfsson, Erik, Tom Mitchell, and Daniel Rock. 2019. Machine learning and occupational change. Unpublished manuscript, Stanford University.

Carnevale, Anthony, Tamara Jayasundera, and Dmitri Repnikov. 2014. Understanding job ads data: a technical report. Unpublished manuscript, Center on Education and the Workforce McCourt School of Public Policy Georgetown University.

Deming, David J., and Kadeem Noray. 2020. Earnings dynamics, changing job skills, and STEM careers. Quarterly Journal of Economics 134(4):1965-2005.

Dixon, Jay, Bryan Hong, and Lynn Wu. 2019. The employment consequences of robots: firm-level evidence. Unpublished manuscript, Statistics Canada.

Felten, Edward, Manav Raj, and Robert Seamans. 2018. A method to link advances in artificial intelligence to occupational abilities. AEA Papers and Proceedings 108:54-57.

Felten, Edward, Manav Raj, and Robert Seamans. 2019. The effect of artificial intelligence on human labor: An ability-based approach. Academy of Management Proceedings 1.

Ford, Martin. 2015. The rise of the robots. New York: Basic Books. 
Graetz, Georg, and Guy Michaels. 2018. Robots at work. The Review of Economics and Statistics 100(5): 753-768.

Grennan, Jillian, and Roni Michaely. 2019. Artificial intelligence and high-skilled work: Evidence from analysts. Unpublished manuscript, Duke University.

Goos, Maarten, and Alan Manning. 2007. Lousy and lovely jobs: The rising polarization of work in Britain. The Review of Economics and Statistics 89(1): 118-133.

Goos, Maarten, Alan Manning, and Anna Salomons. 2014. Explaining job polarization: Routine-biased technological change and offshoring. American Economic Review 104(8): 2509-2526.

Hazell, Jonathon, and Bledi Taska. 2019. Downward rigidity in the wage for new hires. Unpublished manuscript, London School of Economics.

Hershbein, Brad, and Lisa B. Kahn. 2018. Do recessions accelerate routine-biased technological change? Evidence from vacancy postings. American Economic Review 108(7): 1737-1772.

Humlum, Anders. 2019. Robot adoption and labor market dynamics. Unpublished manuscript, University of Chicago Booth School of Business.

Koch, Michael, Ilya Manuylov, and Marcel Smolka. 2021 Robots and firms. The Economic Journal 131(638): 2553-2584.

Krueger, Alan. 1993. How computers have changed the wage structure: Evidence from microdata, 1984-1989. The Quarterly Journal of Economics 108(1): 33-60.

McKinsey Global Institute. 2017. Artificial intelligence: The next digital frontier? Unpublished manuscript, McKinsey Global Institute.

Michaels, Guy, Ashwini Natraj, and John Van Reenen. 2014. Has ICT polarized skill demand? Evidence from eleven countries over twenty-five years. Review of Economics and Statistics 96(1): 60-77.

Neapolitan, Richard E., and Xia Jiang. 2018. Artificial intelligence: With an introduction to machine learning. CRC Press, Taylor \& Francis Group.

Russell, Stuart. 2019. Human compatible: Artificial intelligence and the problem of control. United States: Viking.

Susskind, Daniel. 2020. A world without work: Technology, automation and how we should respond. UK: Penguin.

Tolbert, Charles, and Molly Sizer. 1996. US commuting zones and labor market areas: A 1990 update." Unpublished manuscript, Economic Research Service.

Webb, Michael. 2020. The impact of artificial intelligence on the labor market. Unpublished manuscript, Stanford. 
West, Darrell. 2018. The future of work: robots, AI, and automation. Brookings Institution Press. 
Figure 1: Vacancies in Burning Glass and JOLTS

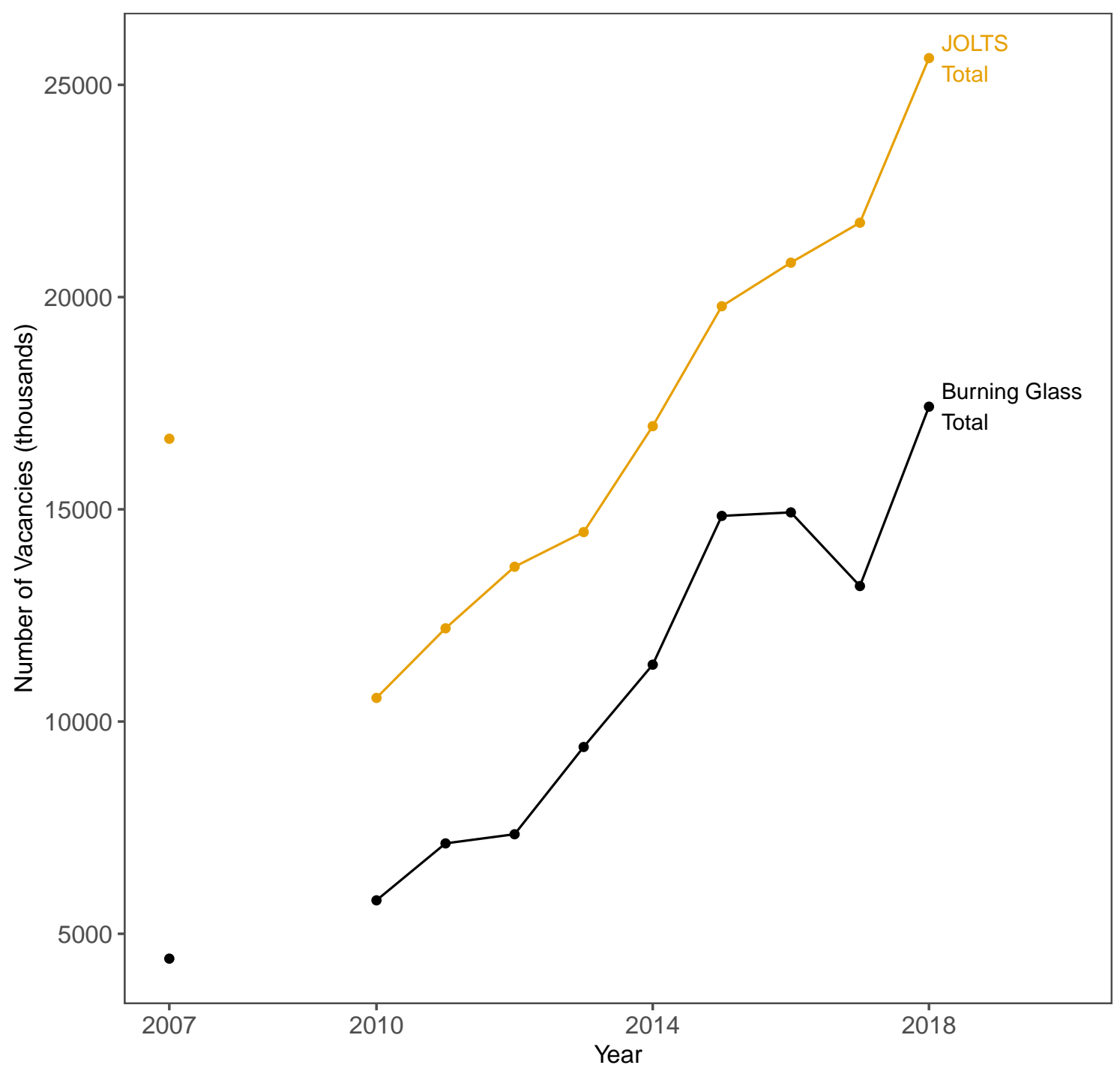

This figure plots the total number of vacancies in JOLTS and the total number of vacancies in Burning Glass by year. We multiply the number of job openings in JOLTS by a constant factor, of 0.65 , to arrive at a number of vacancies that matches the concept of a vacancy in Burning Glass. This method follows Carnevale et al. (2014). 
Figure 2: Share of AI Vacancies in Burning Glass

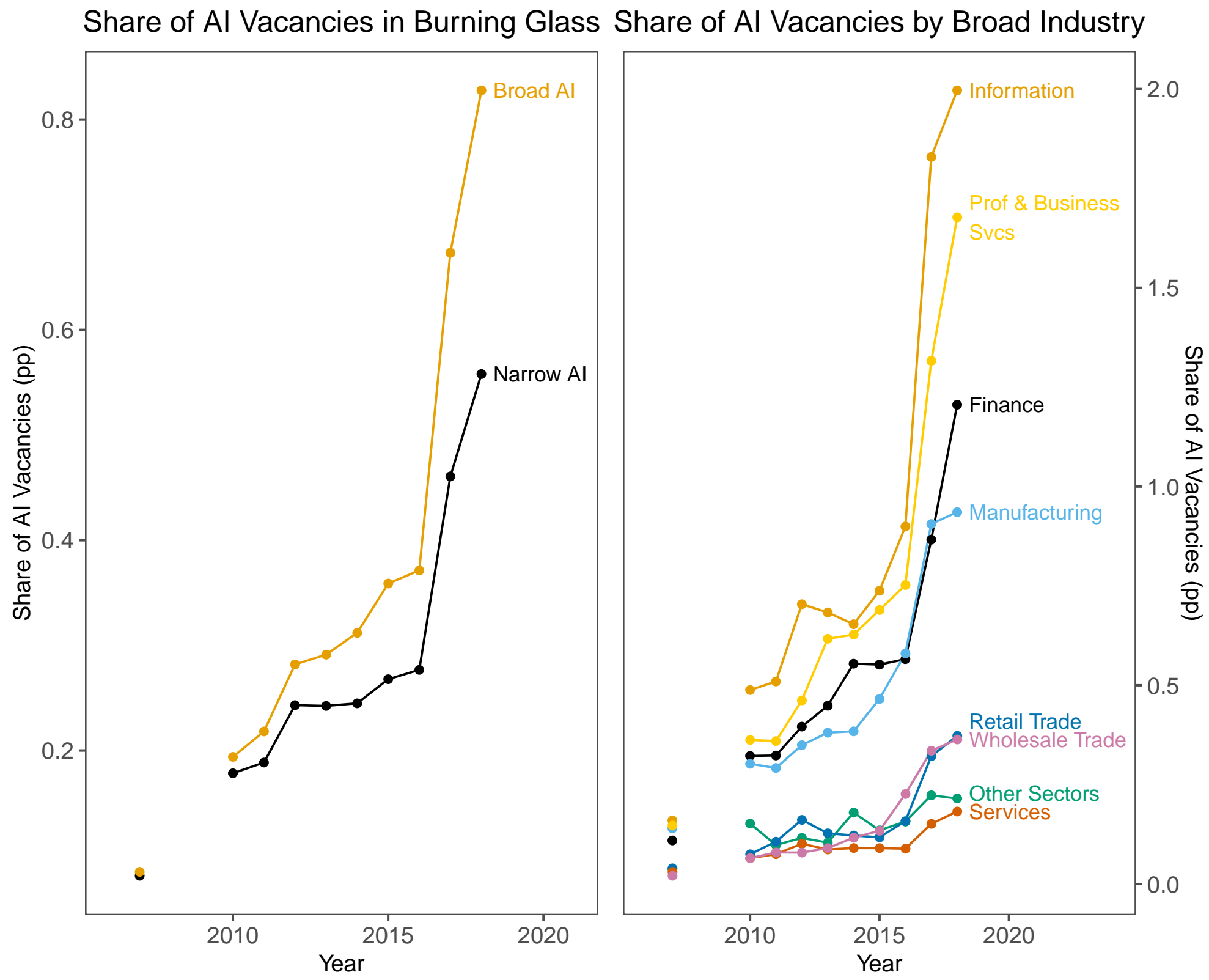

The left panel plots the share of vacancies in Burning Glass that post a skill in the Broad or Narrow AI categories, as defined in the main text. The right panel plots the share of narrow AI vacancies in Burning Glass, by year, in each industry sector. 
Figure 3: AI Exposure by Broad Occupation and Sector

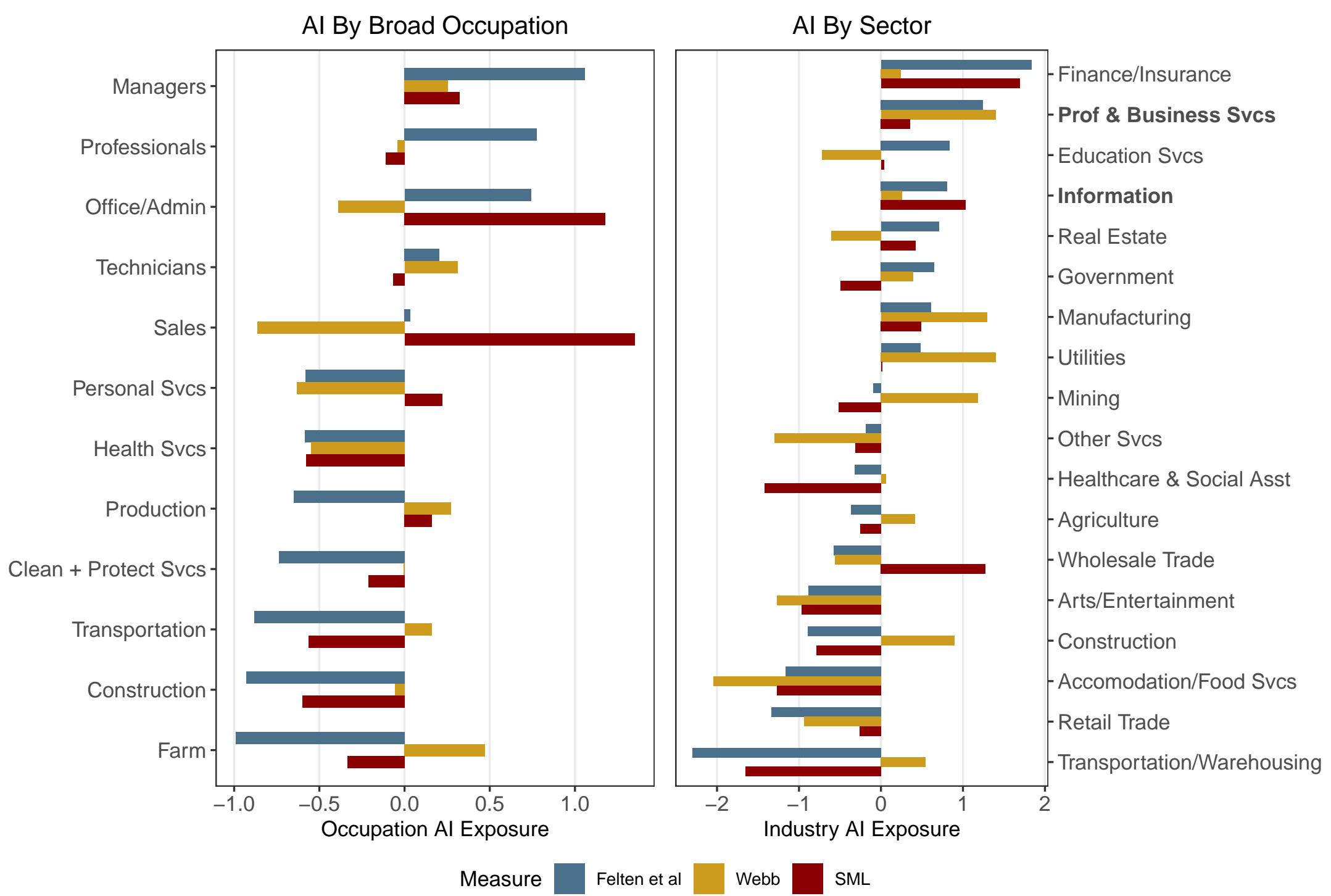

The left panel plots the average of the standardized measures of AI exposure across broad occupations. The right panel plots the average of the standardized measures of AI exposure across 2-digit NAICS sectors, by taking the mean across the 6 digit SOC occupations posted in each 2 digit NAICS sector, weighted by the number of vacancies posted by each sector in each occupation. 


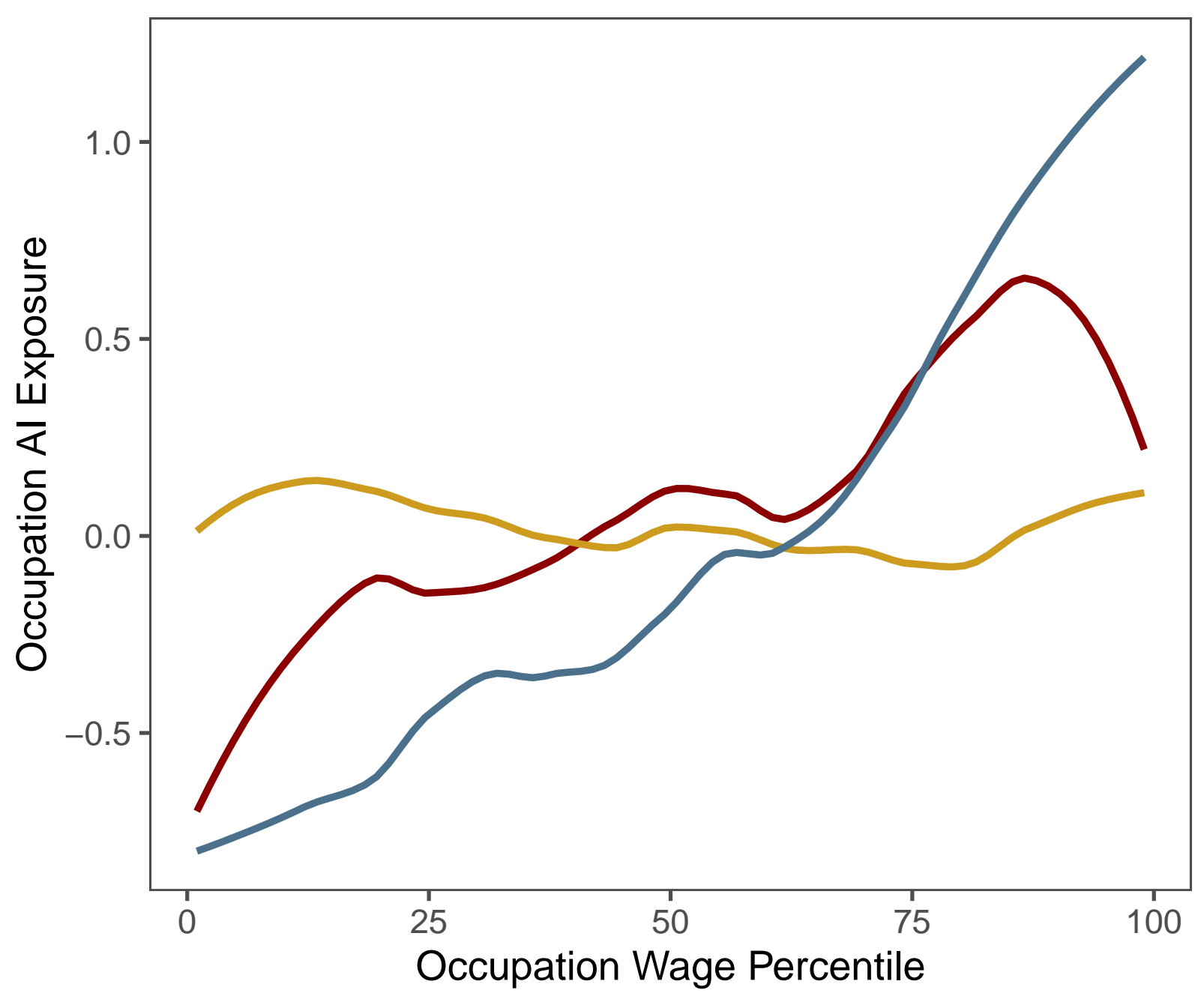

Measure - Felten et al $-\mathrm{SML}-$ Webb

The figure plots a smoothed polynomial regression of the (standardized) measures of AI exposure in each 6-digit SOC occupation against its rank in the wage distribution. We rank occupations according to their mean hourly wage for 2010-2018, obtained from the Occupational Employment Statistics. 
(a) Felten et al Measure

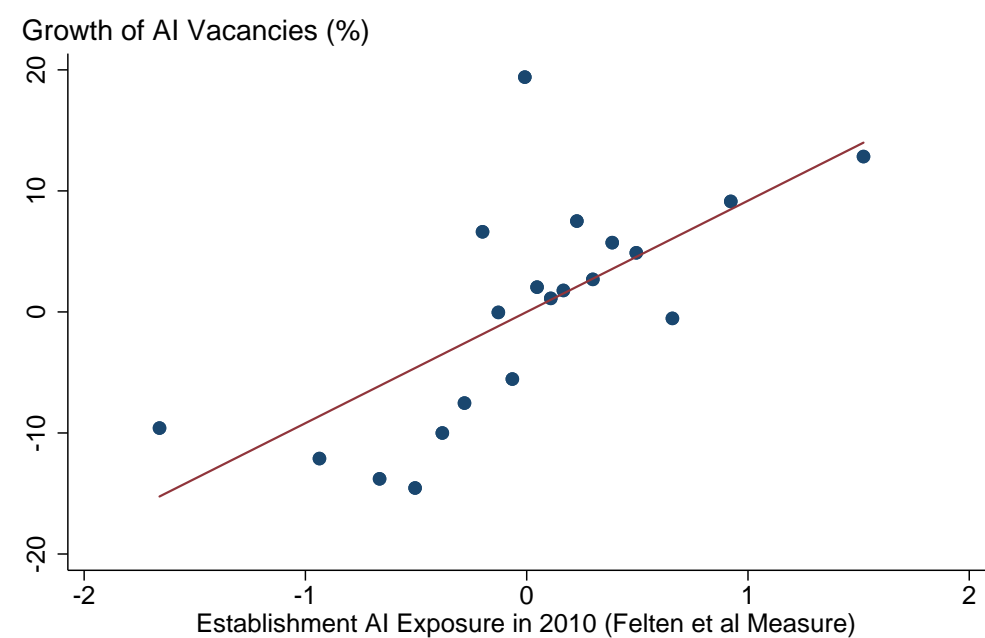

Coefficient is 9.19 , SE is 1.21 , regressor is standardized (b) Webb Measure

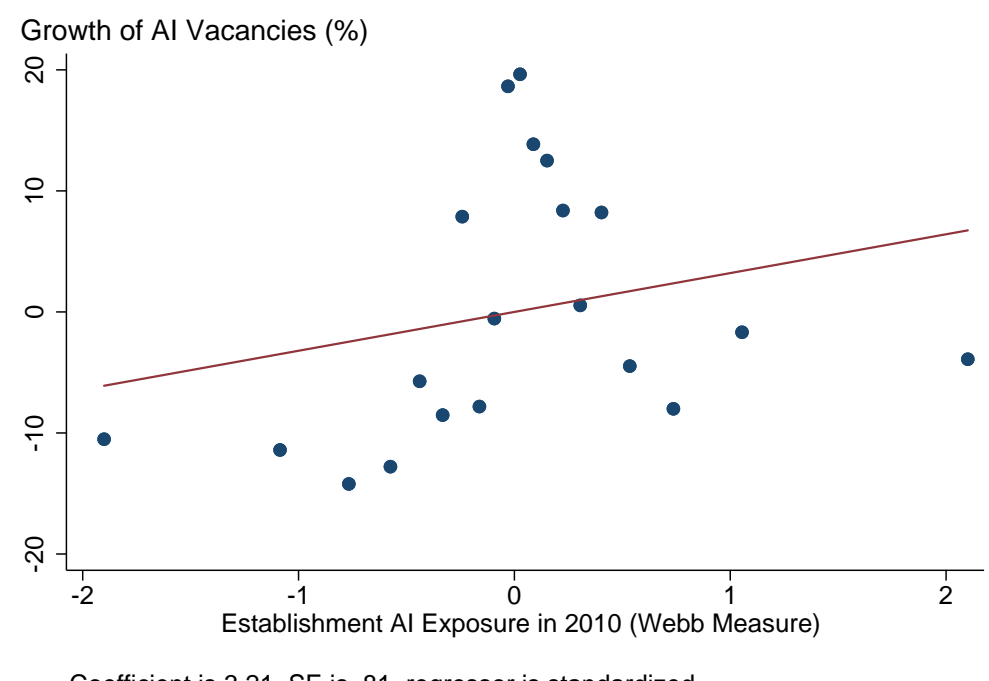

Coefficient is 3.21 , SE is .81 , regressor is standardized

(c) SML Measure

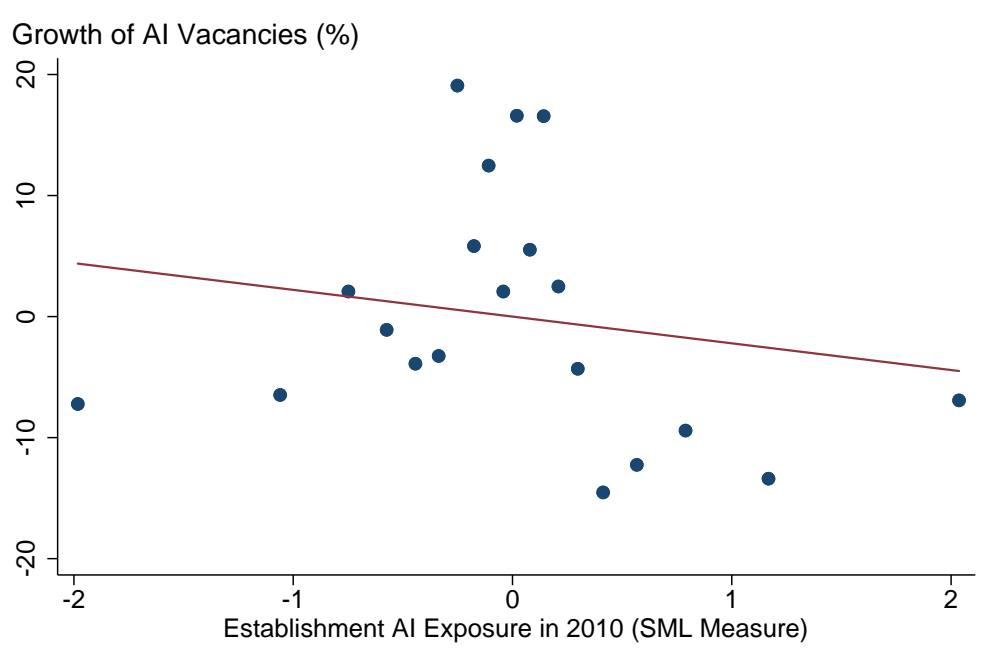

Coefficient is -2.21 , SE is .96 , regressor is standardized

The figure presents binned scatter plots that summarize the relationship between establishment AI Exposure in 2010 and the growth of AI establishment vacancies between 2010 and 2018. Panel A uses the measure of AI exposure from Felten et al. (2019). Panel B uses the Webb (2020) measure. Panel C uses the SML measure, from Brynjolfsson et al. (2019). In all panels, the covariates from column 3 of Table 1 are partialled out. The solid line corresponds to a regression with 2010 establishment vacancies as the weight. The corresponding point estimates and standard errors are reported at the bottom of each panel. We exclude vacancies in industry sectors 51 (information) and 54 (business services). 
FiguRE 6: Establishment Share of AI Vacancies by Quartile of AI Exposure
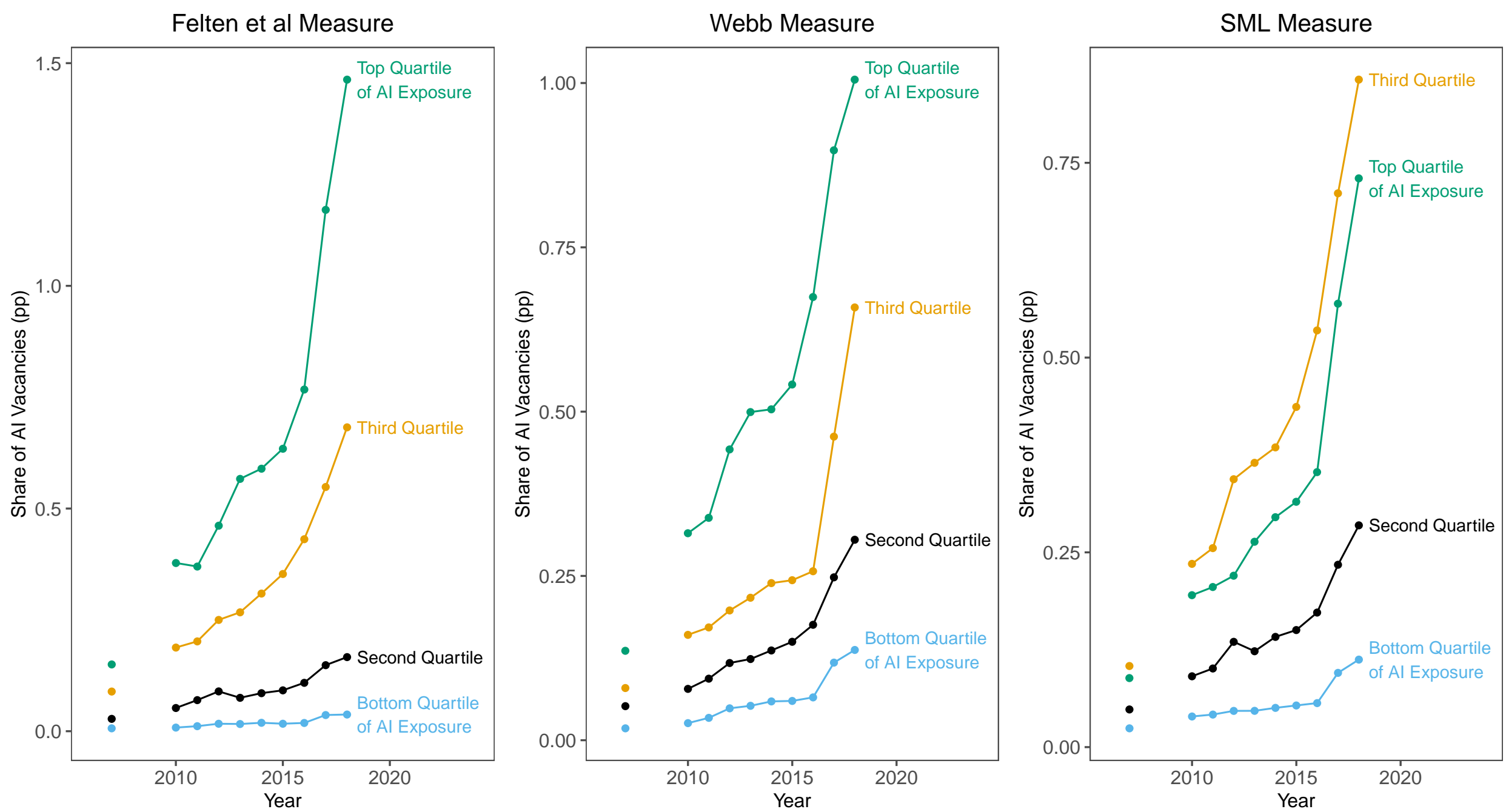

This figure plots establishments' share of AI vacancies in Burning Glass, for each quartile of the distribution of 2010 establishment AI exposure, after partialling out their 2010-2012 share of vacancies in sales and administration. In the first panel, the measure of occupation AI exposure is from Felten et al. (2019). In the second panel, the measure is SML, from Brynjolfsson et al. (2019). In the third panel, the measure is from Webb (2020). We exclude vacancies in industry sectors 51 (information) and 54 (business services). 
Figure 7: Effect of Establishment AI Exposure on Negative Skill Change, by Skill Family
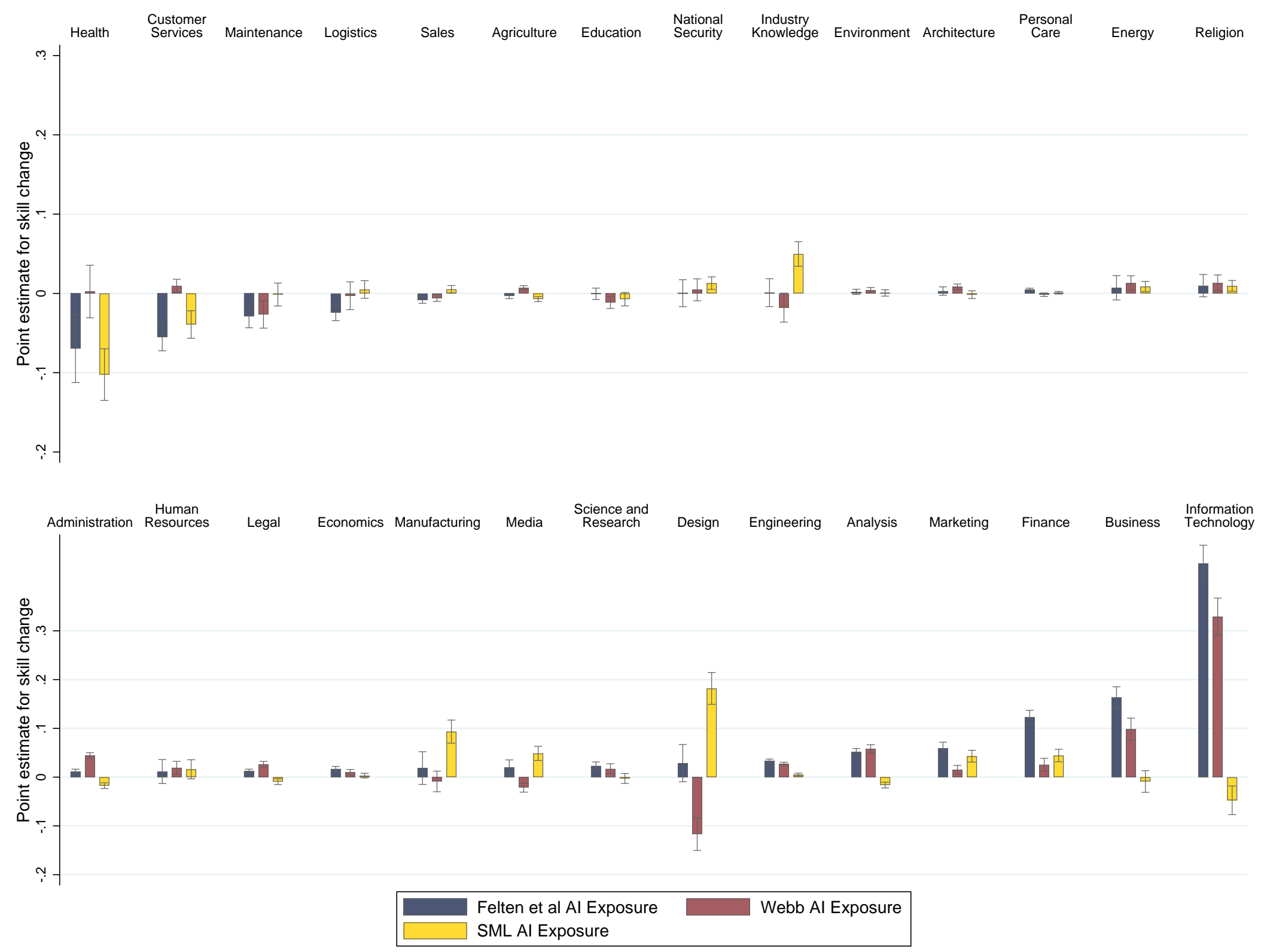

Notes: this figure plots the effect of establishment AI exposure on negative skill change by skill family. We construct measures of negative skill change at the establishment level as defined in the main text, separately for each skill family in Burning Glass. Then we regress establishment negative skill change on establishment AI exposure, using the specification of column (3), Table 3, separately for each skill family. We plot the resulting point estimates and $95 \%$ confidence intervals for our three measures of AI exposure. We list the results separately for each skill family, as described at the top of the graph. We report estimates using our AI exposure measures based on Felten et al. Webb and SML with bars of different shades. 
Figure 8: Effect of Establishment AI Exposure on Positive Skill Change, by Skill Family
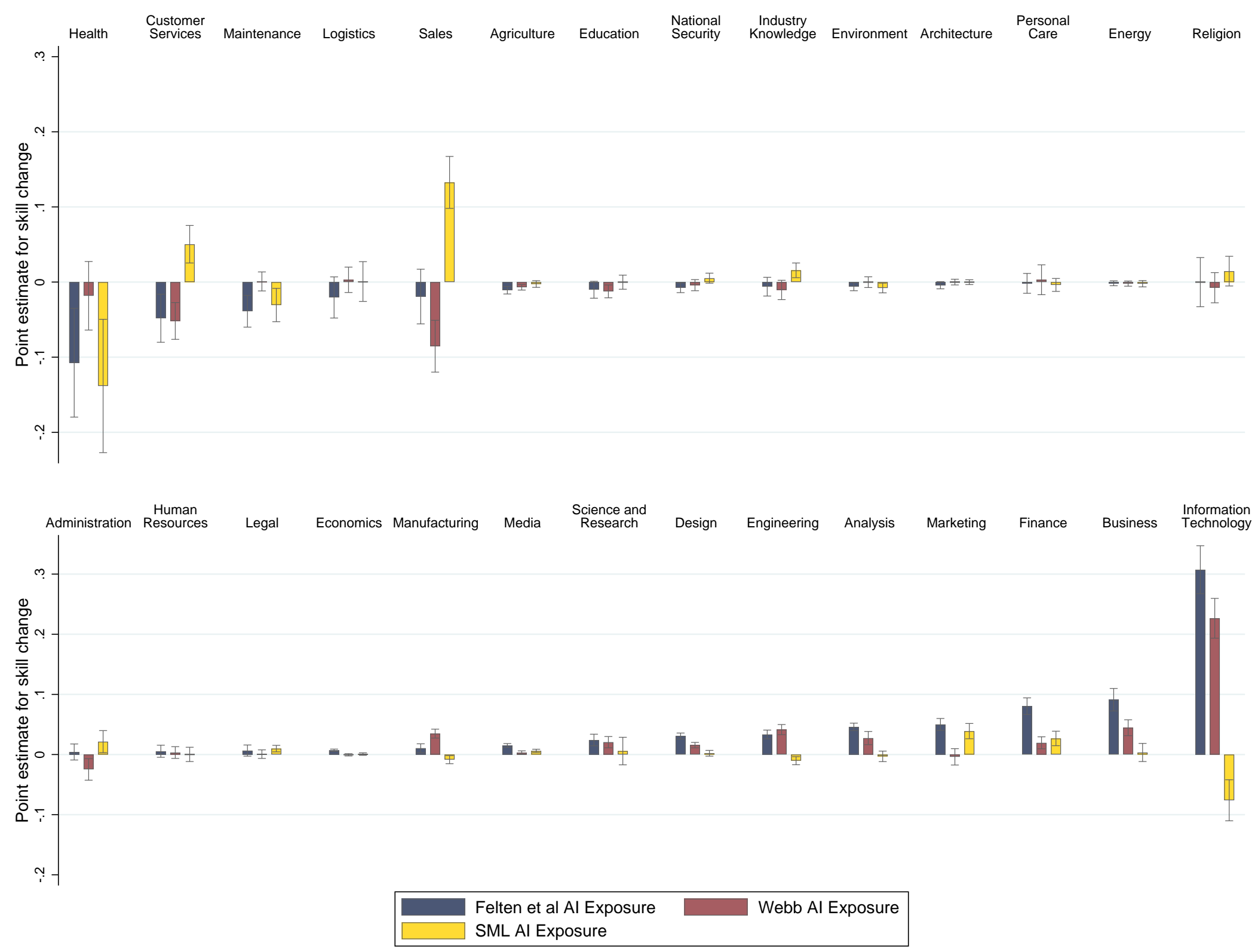

Notes: this figure plots the effect of establishment AI exposure on positive skill change by skill family. We construct measures of positive skill change, at the establishment level as defined in the main text, separately for each skill family in Burning Glass. Then we regress establishment positive skill change on establishment AI exposure, using the specification of column (3), Table 3, separately for each skill family. We plot the resulting point estimates and $95 \%$ confidence intervals for our three measures of AI exposure. We list the results separately for each skill family, as described at the top of the graph. We report estimates using our AI exposure measures based on Felten et al. Webb and SML with bars of different shades. 
TABLE 1: Effects of AI Exposure on Establishment AI Vacancy Growth, 2010-2018

\begin{tabular}{|c|c|c|c|c|c|c|}
\hline & \multicolumn{6}{|c|}{ Growth of Establishment AI Vacancies, 2010-2018 } \\
\hline & $(1)$ & $(2)$ & $(3)$ & $(4)$ & $(5)$ & $(6)$ \\
\hline & \multicolumn{6}{|c|}{ Panel A: Felten et al. Measure of AI Exposure } \\
\hline Establishment AI & 15.96 & 13.82 & 9.19 & 16.53 & 9.75 & 16.87 \\
\hline Exposure, 2010 & $(1.73)$ & $(1.43)$ & $(1.21)$ & $(1.89)$ & $(1.20)$ & $(1.86)$ \\
\hline \multirow[t]{2}{*}{ Observations } & $1,075,474$ & $1,075,474$ & 954,519 & $1,075,474$ & 954,518 & 762,672 \\
\hline & \multicolumn{6}{|c|}{ Panel B: Webb Measure of AI Exposure } \\
\hline Establishment AI & 6.59 & 5.08 & 3.21 & 5.91 & 0.42 & 1.14 \\
\hline Exposure, 2010 & $(1.13)$ & $(0.96)$ & $(0.81)$ & $(1.27)$ & $(0.82)$ & $(1.08)$ \\
\hline \multirow[t]{2}{*}{ Observations } & $1,159,789$ & $1,159,789$ & $1,021,673$ & $1,159,789$ & $1,021,673$ & 824,803 \\
\hline & \multicolumn{6}{|c|}{ Panel C: SML Measure of AI Exposure } \\
\hline Establishment AI & 3.76 & 2.30 & -2.21 & -3.04 & 1.95 & 4.47 \\
\hline Exposure, 2010 & $(1.19)$ & $(1.04)$ & $(0.96)$ & $(1.38)$ & $(0.89)$ & $(1.34)$ \\
\hline Observations & $1,159,789$ & $1,159,789$ & $1,021,673$ & $1,159,789$ & $1,021,673$ & 824,803 \\
\hline \multicolumn{7}{|l|}{ Covariates: } \\
\hline $\begin{array}{l}\text { Share of Vacancies in } \\
\text { sales \& admin, } 2010\end{array}$ & & & & & $\checkmark$ & $\checkmark$ \\
\hline \multicolumn{7}{|l|}{ Fixed Effects: } \\
\hline Firm Size Decile & & $\checkmark$ & $\checkmark$ & & $\checkmark$ & \\
\hline Commuting Zone & & $\checkmark$ & $\checkmark$ & $\checkmark$ & $\checkmark$ & $\checkmark$ \\
\hline 3-digit Industry & & & $\checkmark$ & & $\checkmark$ & \\
\hline Firm & & & & $\checkmark$ & & $\checkmark$ \\
\hline
\end{tabular}

This table presents estimates of the effects of establishment AI exposure on establishment AI vacancy growth. The sample is establishments posting vacancies in 2010-12 or 2016-18, outside NAICS sectors 51 (information) and 54 (business services). The outcome variable, constructed from Burning Glass data, is the growth rate of AI vacancies between 2010 and 2018, multiplied by 100. We approximate this growth rate with the change in the inverse hyperbolic sine of the number of vacancies posted by the establishment in 2010-12 and 2016-18. The regressor, establishment AI exposure in 2010, is the standardized mean of occupation AI exposure, over the 6 digit SOC occupations for which the establishment posts vacancies in 2010-2012, weighted by the number of vacancies posted per occupation. In Panel A, the measure of occupation AI exposure is from Felten et al. (2019). In Panel B, the measure of occupation AI exposure is SML, from Brynjolfsson et al. (2019). In Panel C, the measure of occupation AI exposure is from Webb (2020). The covariates included in each model are reported at the bottom of the table. Column 1 contains only establishment AI exposure. Columns 2-3 and 5 include fixed effects for the decile of firm size (defined as the total vacancies posted by an establishment's firm in 2010-2012). Columns 2-6 include commuting zone fixed effects. Columns 3 and 5 include 3-digit NAICS industry fixed effects. Columns 4 and 6 include firm fixed effects. Columns 5 and 6 control for the share of 2010-12 vacancies in each establishment, belonging to the broad occupations of either sales or administration. In each regression, observations are weighted by total establishment vacancies in 2010-2012. Standard errors are clustered by firm. 
TABLE 2: Effects of AI Exposure on Establishment AI Vacancy Growth, Controlling for Software Exposure

\begin{tabular}{|c|c|c|c|c|c|c|}
\hline & \multicolumn{6}{|c|}{ Growth of Establishment AI Vacancies, 2010-2018 } \\
\hline & (1) & $(2)$ & $(3)$ & $(4)$ & $(5)$ & $(6)$ \\
\hline & \multicolumn{6}{|c|}{ Panel A: Felten et al. Measure of AI Exposure } \\
\hline Establishment AI & 16.28 & 14.10 & 9.63 & 17.43 & 9.96 & 17.47 \\
\hline Exposure, 2010 & $(1.74)$ & $(1.44)$ & $(1.23)$ & $(1.95)$ & $(1.24)$ & $(1.90)$ \\
\hline Estab. Software & 2.36 & 2.24 & 2.62 & 4.83 & 0.66 & 1.83 \\
\hline Exposure & $(0.76)$ & $(0.71)$ & $(0.76)$ & $(1.23)$ & $(0.82)$ & $(1.19)$ \\
\hline \multirow[t]{2}{*}{ Observations } & $1,059,620$ & $1,059,620$ & 941,046 & $1,059,620$ & 941,046 & $1,059,620$ \\
\hline & \multicolumn{6}{|c|}{ Panel B: Webb Measure of AI Exposure } \\
\hline Establishment AI & 10.64 & 7.88 & 3.85 & 6.81 & 1.50 & 2.57 \\
\hline Exposure, 2010 & $(1.83)$ & $(1.50)$ & $(1.10)$ & $(1.52)$ & $(1.07)$ & $(1.41)$ \\
\hline Estab. Software & -6.28 & -4.27 & -0.96 & -1.44 & -1.81 & -2.54 \\
\hline Exposure & $(1.51)$ & $(1.26)$ & $(0.96)$ & $(1.34)$ & $(1.00)$ & $(1.49)$ \\
\hline \multirow[t]{2}{*}{ Observations } & $1,159,789$ & $1,159,789$ & $1,021,673$ & $1,159,789$ & $1,021,673$ & $1,159,789$ \\
\hline & \multicolumn{6}{|c|}{ Panel C: SML Measure of AI Exposure } \\
\hline Establishment AI & 4.14 & 2.64 & -1.96 & -2.42 & 1.90 & 4.37 \\
\hline Exposure, 2010 & $(1.18)$ & $(1.04)$ & $(0.96)$ & $(1.28)$ & $(0.88)$ & $(1.28)$ \\
\hline Estab. Software & 1.56 & 1.44 & 1.09 & 2.40 & -0.90 & -0.76 \\
\hline Exposure & $(0.80)$ & $(0.77)$ & $(0.69)$ & $(1.04)$ & $(0.78)$ & $(1.11)$ \\
\hline Observations & $1,159,789$ & $1,159,789$ & $1,021,673$ & $1,159,789$ & $1,021,673$ & $1,159,789$ \\
\hline \multicolumn{7}{|l|}{ Covariates: } \\
\hline $\begin{array}{l}\text { Share of Vacancies in } \\
\text { sales \& admin, } 2010\end{array}$ & & & & & $\checkmark$ & $\checkmark$ \\
\hline \multicolumn{7}{|l|}{ Fixed Effects: } \\
\hline Firm Size Decile & & $\checkmark$ & $\checkmark$ & & $\checkmark$ & \\
\hline Commuting Zone & & $\checkmark$ & $\checkmark$ & $\checkmark$ & $\checkmark$ & $\checkmark$ \\
\hline 3-digit Industry & & & $\checkmark$ & & $\checkmark$ & \\
\hline Firm & & & & $\checkmark$ & & $\checkmark$ \\
\hline
\end{tabular}

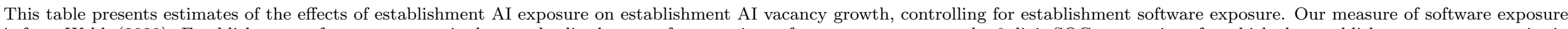

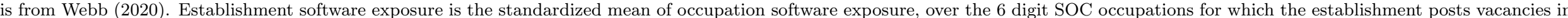

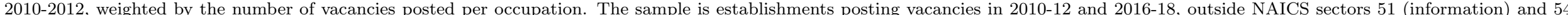

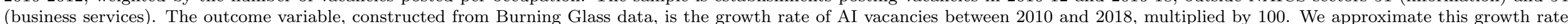

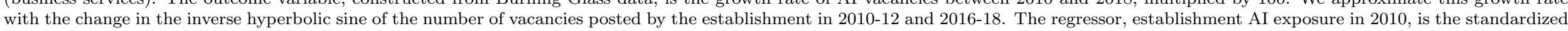

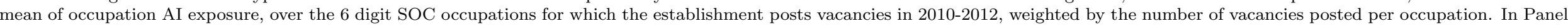

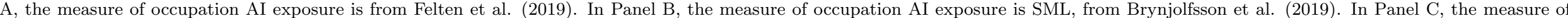

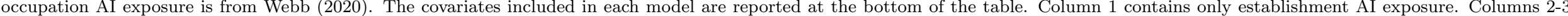

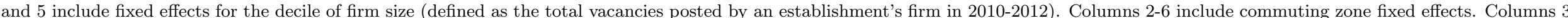

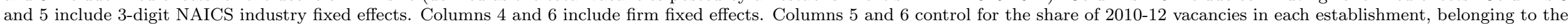

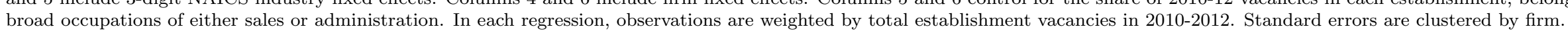


TABLE 3: Effects of AI Exposure on Establishment Negative Skill Change, 2010-2018

Establishment Negative Skill Change, 2010-2018

(1)

(2)

Panel A: Felten et al. Measure of AI Exposure

Establishment AI

Exposure, 2010

Observations

(4)

(5)

(6)

\begin{abstract}
Establishment AI
Exposure, 2010

Observations
\end{abstract}

Establishment AI
Exposure, 2010
Observations

\section{Covariates:}

Share of Vacancies in

sales \& admin, 2010

Fixed Effects:

Firm Size Decile

Commuting Zone

3-digit Industry

Firm
0.83

(0.09)

339,282

0.83

(0.09)

339,282

0.97
$(0.07)$
322,901

0.50

(0.05)

339,282

Panel B: Webb Measure of AI Exposure

$\begin{array}{cc}0.62 & 0.60 \\ (0.11) & (0.11) \\ 353,107 & 353,107\end{array}$

0.45
$(0.06)$

0.20

(0.04)

335,589

353,107

1.00
$(0.07)$
322,901

0.54

339,282

$0.07)$
22,901

0.68

(0.11)

335,589

0.34

353,107

Panel C: SML Measure of AI Exposure

$\begin{array}{cc}0.53 & 0.52 \\ (0.08) & (0.07) \\ 353,107 & 353,107\end{array}$

0.26
$(0.04)$
353,107

335,589
0.46

(0.09)

335,589

353,107

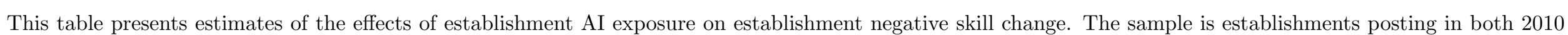

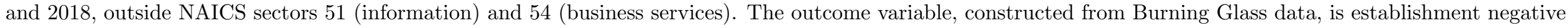

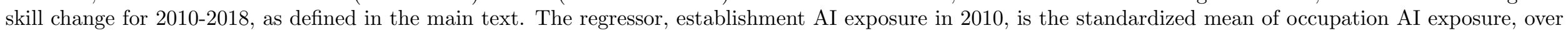

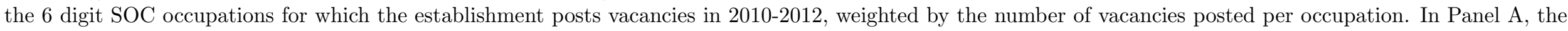

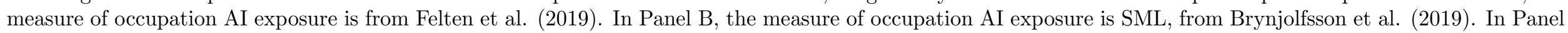

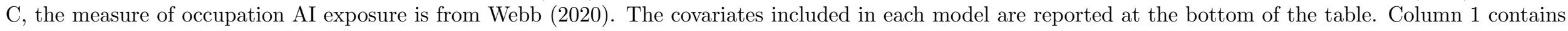

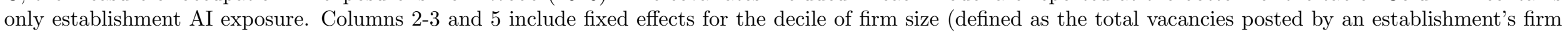

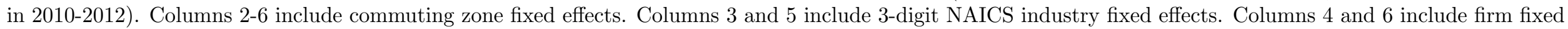

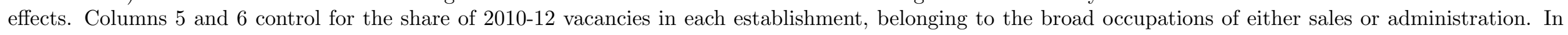
each regression, observations are weighted by total establishment vacancies in 2010-2012. Standard errors are clustered by firm. 
TABLE 4: Effects of AI Exposure on Establishment Positive Skill Change, 2010-2018

\begin{tabular}{|c|c|c|c|c|c|c|}
\hline & \multicolumn{6}{|c|}{ Establishment Positive Skill Change, 2010-2018 } \\
\hline & (1) & $(2)$ & $(3)$ & (4) & $(5)$ & (6) \\
\hline & \multicolumn{6}{|c|}{ Panel A: Felten et al. Measure of AI Exposure } \\
\hline Establishment AI & 0.95 & 0.94 & 0.58 & 0.02 & 0.62 & 0.05 \\
\hline Exposure, 2010 & $(0.08)$ & $(0.09)$ & $(0.09)$ & $(0.04)$ & $(0.09)$ & $(0.04)$ \\
\hline \multirow[t]{2}{*}{ Observations } & 339,282 & 339,282 & 322,901 & 339,282 & 322,901 & 339,282 \\
\hline & \multicolumn{6}{|c|}{ Panel B: Webb Measure of AI Exposure } \\
\hline Establishment AI & 0.69 & 0.66 & 0.26 & -0.01 & 0.43 & 0.13 \\
\hline Exposure, 2010 & $(0.09)$ & $(0.09)$ & $(0.08)$ & $(0.03)$ & $(0.08)$ & $(0.04)$ \\
\hline \multirow[t]{2}{*}{ Observations } & 353,107 & 353,107 & 335,589 & 353,107 & 335,589 & 353,107 \\
\hline & \multicolumn{6}{|c|}{ Panel C: SML Measure of AI Exposure } \\
\hline Establishment AI & 0.62 & 0.59 & 0.19 & 0.10 & 0.26 & 0.03 \\
\hline Exposure, 2010 & $(0.09)$ & $(0.09)$ & $(0.09)$ & $(0.04)$ & $(0.09)$ & $(0.04)$ \\
\hline Observations & 353,107 & 353,107 & 335,589 & 353,107 & 335,589 & 353,107 \\
\hline \multicolumn{7}{|l|}{ Covariates: } \\
\hline $\begin{array}{l}\text { Share of Vacancies in } \\
\text { sales \& admin, } 2010\end{array}$ & & & & & $\checkmark$ & $\checkmark$ \\
\hline \multicolumn{7}{|l|}{ Fixed Effects: } \\
\hline Firm Size Decile & & $\checkmark$ & $\checkmark$ & & $\checkmark$ & \\
\hline Commuting Zone & & $\checkmark$ & $\checkmark$ & $\checkmark$ & $\checkmark$ & $\checkmark$ \\
\hline 3-digit Industry & & & $\checkmark$ & & $\checkmark$ & \\
\hline Firm & & & & $\checkmark$ & & $\checkmark$ \\
\hline
\end{tabular}

This table presents estimates of the effects of establishment AI exposure on establishment positive skill change. The sample is establishments posting vacancies in 2010-12 and 2016-18, outside NAICS sectors 51 (information) and 54 (business services). The outcome variable, constructed from Burning Glass data, is establishment positive skill change for 2010-2018, as defined in the main text. The sample is establishments posting in both 2010 and 2019 . The regressor, establishment AI exposure in 2010, is the standardized mean of occupation AI exposure, over the 6 digit SOC occupations for which the establishment posts vacancies in 2010-2012, weighted by the number of vacancies posted per occupation. In Panel A, the measure of occupation AI exposure is from Felten et al. (2019). In Panel B, the measure of occupation AI exposure is SML, from Brynjolfsson et al. (2019). In Panel C, the measure of occupation AI exposure is from Webb (2020). The covariates included in each model are reported at the bottom of the table. Column 1 contains only establishment AI exposure. Columns 2-3 and 5 include fixed effects for the decile of firm size (defined as the total vacancies posted by an establishment's firm in 2010-2012). Columns 2-6 include commuting zone fixed effects. Columns 3 and 5 include 3-digit NAICS industry fixed effects. Columns 4 and 6 include firm fixed effects. Columns 5 and 6 control for the share of 2010-12 vacancies in each establishment, belonging to the broad occupations of either sales or administration. In each regression, observations are weighted by total establishment vacancies in 2010-2012. Standard errors are clustered by firm. 
TABLE 5: Effects of AI Exposure on Establishment Non-AI Vacancy Growth, 2010-2018

\begin{tabular}{|c|c|c|c|c|c|c|c|c|}
\hline & \multicolumn{8}{|c|}{ Growth of Establishment Non-AI Vacancies, 2010-2018 } \\
\hline & \multicolumn{6}{|c|}{ Full Sample } & \multicolumn{2}{|c|}{ Establishments Posting in 2018} \\
\hline & $(1)$ & $(2)$ & $(3)$ & $(4)$ & $(5)$ & $(6)$ & $(7)$ & $(8)$ \\
\hline & \multicolumn{8}{|c|}{ Panel A: Felten et al. Measure of AI Exposure } \\
\hline Establishment AI & -13.80 & -16.36 & -11.90 & -4.81 & -12.42 & -4.04 & -8.38 & -3.56 \\
\hline Exposure, 2010 & $(4.22)$ & $(4.11)$ & $(4.08)$ & $(1.44)$ & $(4.01)$ & $(1.47)$ & $(3.46)$ & $(1.86)$ \\
\hline \multirow[t]{2}{*}{ Observations } & $1,075,474$ & $1,075,474$ & 954,519 & $1,075,474$ & 954,519 & $1,075,474$ & 324,901 & 341,525 \\
\hline & \multicolumn{8}{|c|}{ Panel B: Webb Measure of AI Exposure } \\
\hline Establishment AI & -17.24 & -18.21 & -6.73 & -2.22 & -8.30 & 1.51 & -4.70 & -1.44 \\
\hline Exposure, 2010 & $(3.72)$ & $(3.63)$ & $(3.01)$ & $(0.93)$ & $(3.70)$ & $(0.98)$ & $(2.66)$ & $(1.36)$ \\
\hline \multirow[t]{2}{*}{ Observations } & $1,159,789$ & $1,159,789$ & $1,021,673$ & $1,159,789$ & $1,021,673$ & $1,159,789$ & 337,758 & 355,529 \\
\hline & \multicolumn{8}{|c|}{ Panel C: SML Measure of AI Exposure } \\
\hline Establishment AI & 7.02 & 5.74 & 2.05 & 0.95 & 2.21 & -3.01 & 0.01 & -0.91 \\
\hline Observations & $1,159,789$ & $1,159,789$ & $1,021,673$ & $1,159,789$ & $1,021,673$ & $1,159,789$ & 337,758 & 355,529 \\
\hline \multicolumn{9}{|l|}{ Covariates: } \\
\hline Share of Vacancies in & & & & & $\checkmark$ & $\checkmark$ & & \\
\hline \multicolumn{9}{|l|}{ Sales, Admin. in 2010} \\
\hline \multicolumn{9}{|l|}{ Fixed Effects: } \\
\hline Firm Size Decile & & $\checkmark$ & $\checkmark$ & & $\checkmark$ & & $\checkmark$ & \\
\hline Commuting Zone & & $\checkmark$ & $\checkmark$ & $\checkmark$ & $\checkmark$ & $\checkmark$ & $\checkmark$ & $\checkmark$ \\
\hline 3-digit Industry & & & $\checkmark$ & & $\checkmark$ & & $\checkmark$ & \\
\hline Firm & & & & $\checkmark$ & & $\checkmark$ & & $\checkmark$ \\
\hline
\end{tabular}

This table presents estimates of the effects of establishment AI exposure on establishment non-AI vacancy growth. The sample is establishments posting vacancies in 2010-12 and 2016-18, outside NAICS sectors 51 (information) and 54 (business services). The outcome variable, constructed from Burning Glass data, is the growth rate of non-AI vacancies between 2010 and 2018, multiplied by 100. We approximate this growth rate with the change in the inverse hyperbolic sine of the number of vacancies posted by the establishment in 2010-12 and 2016-18. The regressor, establishment AI exposure in 2010, is the standardized mean of occupation AI exposure, over the 6 digit SOC occupations for which the establishment posts vacancies in 2010-2012, weighted by the number of vacancies posted per occupation. In Panel A, the measure of occupation AI exposure is from Felten et al. (2019). In Panel B, the measure of occupation AI exposure is SML, from Brynjolfsson et al. (2019). In Panel C, the measure of occupation AI exposure is from Webb (2020). The final two columns exclude establishments that do not post positive vacancies in 2018. The covariates included in each model are reported at the bottom of the table. Column 1 contains only establishment AI exposure. Columns 2, 3,5 and 7 include fixed effects for the decile of firm size (defined as the total vacancies posted by an establishment's firm in 2010-2012). Columns 2-8 include commuting zone fixed effects. Columns 3, 5 and 7 include 3-digit NAICS industry fixed effects. Columns 4, 6 and 8 include firm fixed effects. Columns 5 and 6 control for the share of 2010-12 vacancies in each establishment, belonging to the broad occupations of either sales or administration. In each regression, observations are weighted by total vacancies in 2010-2012. Standard errors are clustered by firm. 
TABle 6: Effects of AI Exposure on Establishment Non-AI Vacancy Growth, Controlling for Software Exposure

\begin{tabular}{|c|c|c|c|c|c|c|c|c|}
\hline & \multicolumn{8}{|c|}{ Growth of Establishment Non-AI Vacancies, 2010-2018 } \\
\hline & \multicolumn{6}{|c|}{ Full Sample } & \multicolumn{2}{|c|}{ Establishments Posting in 2018} \\
\hline & $(1)$ & $(2)$ & $(3)$ & $(4)$ & $(5)$ & $(6)$ & (7) & (8) \\
\hline & \multicolumn{8}{|c|}{ Panel A: Felten et al. Measure of AI Exposure } \\
\hline Establishment AI & -14.62 & -17.21 & -12.02 & -5.43 & -12.47 & -3.93 & -8.68 & -3.73 \\
\hline Exposure, 2010 & $(4.28)$ & $(4.17)$ & $(4.00)$ & $(1.49)$ & $(3.94)$ & $(1.54)$ & $(3.36)$ & $(1.90)$ \\
\hline Estab. Software & -7.50 & -7.35 & 0.66 & -1.77 & 1.07 & 2.03 & -1.67 & -0.41 \\
\hline Exposure & $(3.68)$ & $(3.60)$ & $(3.09)$ & $(1.08)$ & $(3.28)$ & $(1.17)$ & $(3.41)$ & $(1.40)$ \\
\hline \multirow[t]{2}{*}{ Observations } & $1,059,620$ & $1,059,620$ & 941,046 & $1,059,620$ & 941,046 & $1,059,620$ & 322,187 & 338,645 \\
\hline & \multicolumn{8}{|c|}{ Panel B: Webb Measure of AI Exposure } \\
\hline Establishment AI & -23.04 & -25.36 & -14.04 & -3.06 & -14.95 & -0.29 & -7.30 & -2.56 \\
\hline Exposure, 2010 & $(4.61)$ & $(4.47)$ & $(4.68)$ & $(1.05)$ & $(5.59)$ & $(1.09)$ & $(4.51)$ & $(1.56)$ \\
\hline Estab. Software & 9.01 & 10.88 & 10.98 & 1.36 & 11.16 & 3.19 & 3.80 & 1.74 \\
\hline Exposure & $(4.32)$ & $(4.22)$ & $(4.79)$ & $(1.14)$ & $(5.11)$ & $(1.18)$ & $(5.26)$ & $(1.54)$ \\
\hline \multirow[t]{2}{*}{ Observations } & $1,159,789$ & $1,159,789$ & $1,021,673$ & $1,159,789$ & $1,021,673$ & $1,159,789$ & 337,758 & 355,529 \\
\hline & \multicolumn{8}{|c|}{ Panel C: SML Measure of AI Exposure } \\
\hline Establishment AI & 5.97 & 4.70 & 2.62 & 0.84 & 2.40 & -2.67 & -0.17 & -0.94 \\
\hline Exposure, 2010 & $(3.15)$ & $(3.03)$ & $(2.93)$ & $(1.12)$ & $(3.63)$ & $(1.20)$ & $(2.89)$ & $(1.39)$ \\
\hline Estab. Software & -4.41 & -4.37 & 2.46 & -0.43 & 3.04 & 2.80 & -0.85 & -0.13 \\
\hline Exposure & $(3.66)$ & $(3.60)$ & $(3.10)$ & $(0.95)$ & $(3.30)$ & $(1.03)$ & $(3.36)$ & $(1.34)$ \\
\hline Observations & $1,159,789$ & $1,159,789$ & $1,021,673$ & $1,159,789$ & $1,021,673$ & $1,159,789$ & 337,758 & 355,529 \\
\hline \multicolumn{9}{|l|}{ Covariates: } \\
\hline $\begin{array}{l}\text { Share of Vacancies in } \\
\text { sales \& admin, } 2010 \\
\text { Fixed Effects: }\end{array}$ & & & & & $\checkmark$ & $\checkmark$ & & \\
\hline Firm Size Decile & & $\checkmark$ & $\checkmark$ & & $\checkmark$ & & $\checkmark$ & \\
\hline Commuting Zone & & $\checkmark$ & $\checkmark$ & $\checkmark$ & $\checkmark$ & $\checkmark$ & $\checkmark$ & $\checkmark$ \\
\hline 3-digit Industry & & & $\checkmark$ & & $\checkmark$ & & $\checkmark$ & \\
\hline Firm & & & & $\checkmark$ & & $\checkmark$ & & $\checkmark$ \\
\hline
\end{tabular}

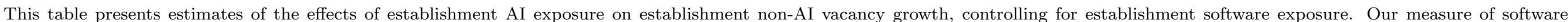

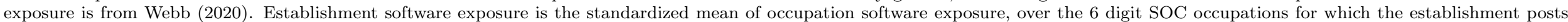

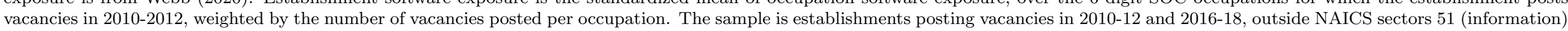

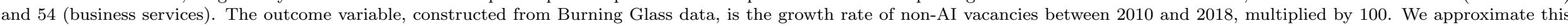

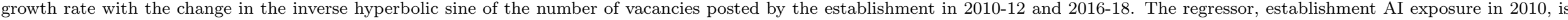

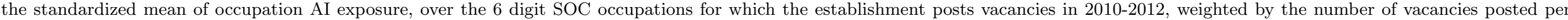

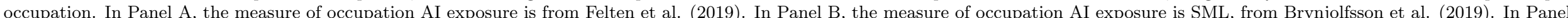

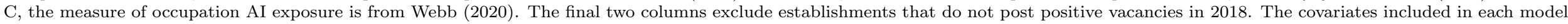

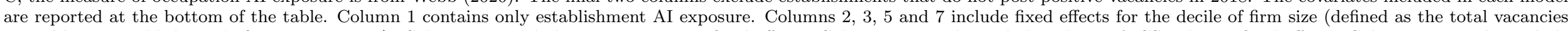

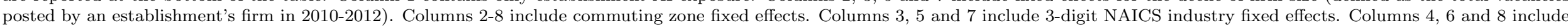

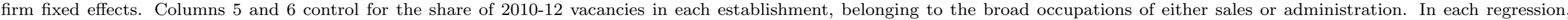
observations are weighted by total vacancies in 2010-2012. Standard errors are clustered by firm. 
TABLE 7: Effects of AI Exposure on Establishment Non-AI Vacancy Growth, 2010-2014 and 2014-2018

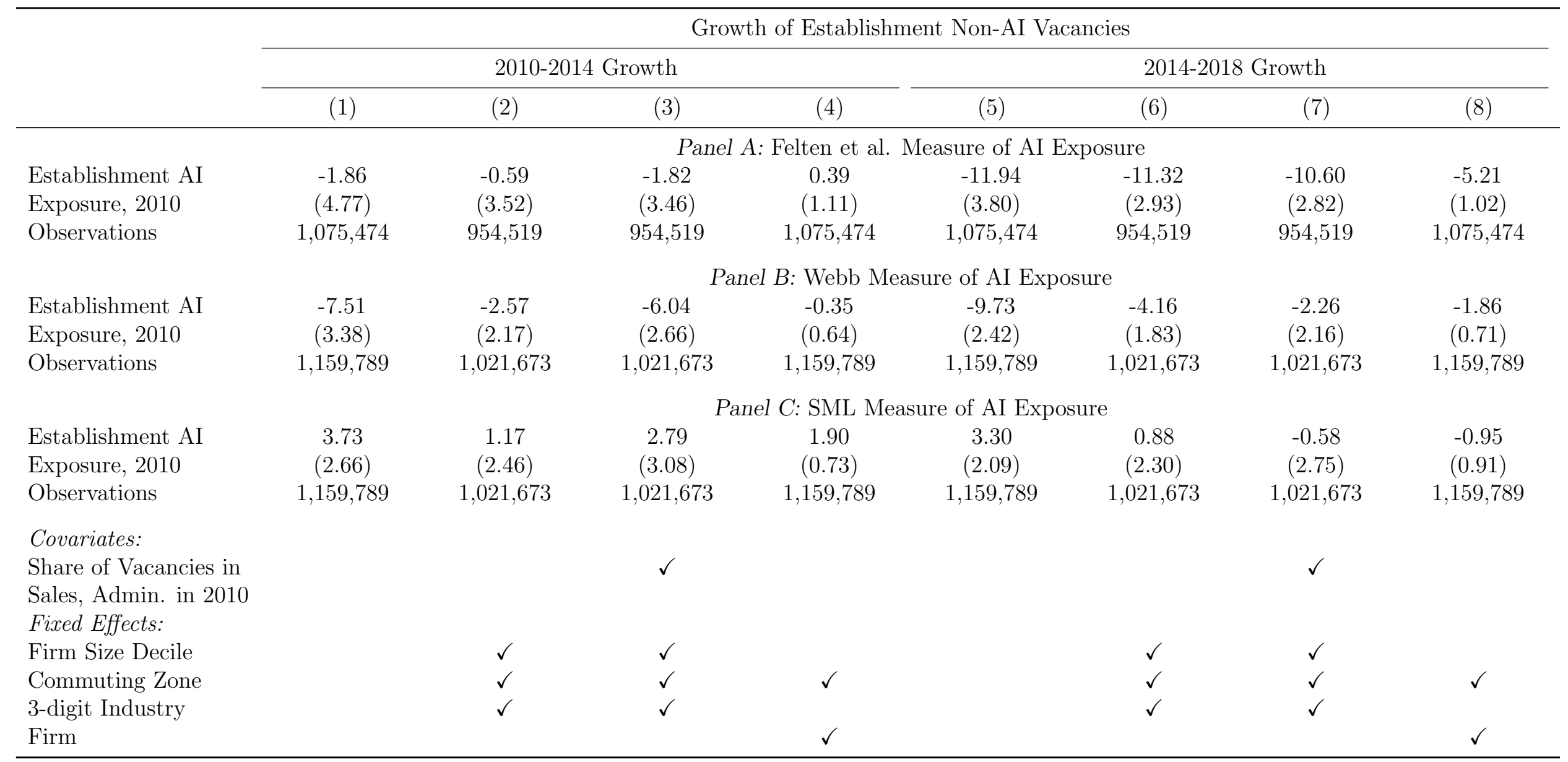

This table presents estimates of the effects of establishment AI exposure on establishment non-AI vacancy growth, separately for 2010-2014 and 2014-2018. The sample is establishments posting vacancies in 2010-12 and 2016-18, outside NAICS sectors 51 (information) and 54 (business services). In columns 1-4, the outcome variable, constructed from Burning Glass data, is the growth rate of non-AI vacancies between 2010 and 2014, multiplied by 100. We approximate this growth rate with the change in the inverse hyperbolic sine of the number of vacancies posted by the establishment in 2010-12 and 2013-15. In columns 5-8, the outcome variable is the change in the inverse hyperbolic sine of the number of vacancies posted by the establishment in 2013-15 and 2016-18. The regressor, establishment AI exposure in 2010, is the standardized mean of occupation AI exposure, over the 6 digit SOC occupations for which the establishment posts vacancies in 2010-2012, weighted by the number of vacancies posted per occupation. In Panel A, the measure of occupation AI exposure is from Felten et al. (2019). In Panel B, the measure of occupation AI exposure is SML, from Brynjolfsson et al. (2019). In Panel C, the measure of occupation AI exposure is from Webb (2020). The covariates included in each model are reported at the bottom of the table. Columns 1 and 5 contains only establishment AI exposure. Columns $2,3,6$ and 7 include fixed effects for the decile of firm size (defined as the total vacancies posted by an establishment's firm in 2010-2012); and also include 3-digit NAICS industry fixed effects. All columns other than 1 and 5 include commuting zone fixed effects. Columns 4 and 8 include firm fixed effects. Columns 3 and 7 control for the share of $2010-12$ vacancies in each establishment, belonging to the broad occupations of either sales or administration. In each regression, observations are weighted by total vacancies in $2010-2012$. Standard errors are clustered by firm. 
TABLE 8: Effects of AI Exposure on Market Employment and Wage Growth

\begin{tabular}{|c|c|c|c|c|c|c|c|c|c|}
\hline & \multicolumn{3}{|c|}{ Industry by CZ Employment Growth (CBP) } & \multicolumn{3}{|c|}{ Occupation Employment Growth (OES) } & \multicolumn{3}{|c|}{ Occupation Wage Growth (OES) } \\
\hline & $\begin{array}{l}2003-2007 \\
\quad(1)\end{array}$ & $\begin{array}{l}2007-2010 \\
(2)\end{array}$ & $\begin{array}{c}2010-2016 \\
(3)\end{array}$ & $\begin{array}{c}2004-2007 \\
(4)\end{array}$ & $\begin{array}{l}2007-2010 \\
\quad(5)\end{array}$ & $\begin{array}{c}2010-2018 \\
(6)\end{array}$ & $\begin{array}{l}2004-2007 \\
\quad(7)\end{array}$ & $\begin{array}{l}2007-2010 \\
\quad(8)\end{array}$ & $\begin{array}{c}2010-2018 \\
(9)\end{array}$ \\
\hline & \multicolumn{9}{|c|}{ Panel A: Felten et al. Measure of AI Exposure } \\
\hline Market AI Exposure, & 0.03 & 0.10 & -0.05 & 0.34 & 0.86 & 0.51 & -0.00 & 0.02 & -0.17 \\
\hline 2010 & $(0.17)$ & $(0.20)$ & $(0.08)$ & $(0.34)$ & $(0.32)$ & $(0.35)$ & $(0.17)$ & $(0.20)$ & $(0.06)$ \\
\hline \multirow[t]{2}{*}{ Observations } & 10,937 & 10,926 & 10,929 & 736 & 700 & 680 & 680 & 648 & 629 \\
\hline & \multicolumn{9}{|c|}{ Panel B: Webb Measure of AI Exposure } \\
\hline Market AI Exposure, & 0.10 & 0.18 & 0.11 & 0.00 & 0.11 & -0.17 & 0.11 & -0.05 & -0.02 \\
\hline 2010 & $(0.15)$ & $(0.17)$ & $(0.09)$ & $(0.17)$ & $(0.21)$ & $(0.29)$ & $(0.08)$ & $(0.10)$ & $(0.04)$ \\
\hline \multirow[t]{2}{*}{ Observations } & 10,981 & 10,968 & 10,968 & 713 & 704 & 717 & 660 & 653 & 663 \\
\hline & \multicolumn{9}{|c|}{ Panel $C$ : SML Measure of AI Exposure } \\
\hline Market AI Exposure, & -0.14 & 0.37 & -0.01 & 0.00 & -0.17 & -0.37 & -0.03 & 0.18 & 0.04 \\
\hline 2010 & $(0.17)$ & $(0.18)$ & $(0.08)$ & $(0.25)$ & $(0.29)$ & $(0.25)$ & $(0.08)$ & $(0.12)$ & $(0.05)$ \\
\hline Observations & 10,981 & 10,968 & 10,968 & 713 & 704 & 717 & 660 & 653 & 663 \\
\hline \multicolumn{10}{|l|}{ Covariates: } \\
\hline Share of Vacancies in & $\checkmark$ & $\checkmark$ & $\checkmark$ & & & & & & \\
\hline \multirow{2}{*}{\multicolumn{10}{|c|}{$\begin{array}{l}\text { Sales, Admin. in } 2010 \\
\text { Fixed Effects: }\end{array}$}} \\
\hline & & & & & & & & & \\
\hline Commuting Zone & $\checkmark$ & $\checkmark$ & $\checkmark$ & & & & & & \\
\hline Sector & $\checkmark$ & $\checkmark$ & $\checkmark$ & & & & & & \\
\hline 3-digit Occupation & & & & $\checkmark$ & $\checkmark$ & $\checkmark$ & $\checkmark$ & $\checkmark$ & $\checkmark$ \\
\hline
\end{tabular}

This table presents estimates of the effects of market AI exposure on market employment and wage growth. In columns 1-3, the outcome is the growth rate of sector (i.e. 2 digit NAICS industry) by commuting zone employment, measured in percentage points per year (i.e. $100 \mathrm{x}$ the log change divided by number of years), from the County Business Patterns; for 2003-2007, 2007-2010 and 2010-2016, respectively. The sample excludes industry sectors 51 (information) and 54 (business services). In columns 4-6, the outcome is the growth rate of 6 digit SOC occupation employment outside sectors 51 and 54 , measured in percentage points per year, from the Occupation Employment Statistics; for 2004-2007, 2007-2010 and 2010-2018, respectively. In columns 7-9, the outcome is the growth of 6 digit SOC median hourly wages outside sectors 51 and 54, measured in percentage points per year, also from the Occupational Employment Statistics. In columns 1-3, the regressor is the standardized mean occupation AI exposure, across the 6 digit occupations posted in each sector by commuting zone cell, based on the distribution of vacancies by detailed occupation in each zone and industry in 2010-2012. The regressions are weighted by baseline employment in each sector by commuting zone. In columns 4-9, the regressor is standardized occupation AI exposure by 6 digit SOC occupation. In panel A, the measure of occupation AI exposure is from Felten et al. (2019); in panel B the measure is SML from Brynjolfsson et al. (2019); in panel C the measure is from Webb (2020). All regressions are weighted by baseline employment. The covariates included in each model are reported at the bottom of the table. Columns 1-3 contain sector and commuting zone fixed effects, and controls for the share of 2010-2012 vacancies in either sales or administration in each sector by commuting zone, measured from Burning Glass. Columns 4-9 control for 3-digit SOC occupation fixed effects. Standard errors are clustered by commuting zone in columns 1-3, and robust against heteroskedasticity in columns 4-9. 


\title{
AI AND Jobs: Evidence From OnLINe VaCancies (Online Appendix)
}

\author{
Daron Acemoglu David Autor Jonathon Hazell \\ MIT and NBER MIT and NBER LSE \\ Pascual Restrepo \\ Boston University and NBER
}

October 2021 


\section{Appendix A: Proofs}

Proofs of Propositions 1 and 2:. Consider an establishment $e$ that initially allocates tasks $\mathcal{T}_{e}^{N}$ to (non-AI) labor and $\mathcal{T}_{e}^{A I}$ to AI algorithms. The unit cost of production for this establishment is

$\ln C_{e}=\int_{\mathcal{T}_{e}^{N}} \alpha(x) \ln \left(w / \gamma^{\ell}(x)\right) d x+\int_{\mathcal{T}_{e}^{A I}} \alpha(x) \ln \left(\left(w^{A I}+w^{K}\right) / \gamma^{a}(x)\right) d x-\int_{\mathcal{T}_{e}} \alpha(x) \ln (\alpha(x)) d x$.

Moreover, its total demand for non-AI workers can be computed as

$$
\ell_{e}^{N}=\frac{1}{w^{N}} \cdot y_{e} \cdot C_{e} \cdot \int_{\mathcal{T}_{e}^{N}} \alpha(x) d x,
$$

and the total demand for AI workers can be computed as

$$
\ell_{e}^{A I}=\frac{1}{w^{A I}+w^{K}} \cdot y_{e} \cdot C_{e} \cdot \int_{\mathcal{T}_{e}^{A I}} \alpha(x) d x
$$

Consider a (discrete) improvement in the productivity of algorithms for an infinitesimal set of tasks $\mathcal{T}^{A}$, so that it becomes cheaper to perform these tasks by algorithms rather than workers.

As stated in both propositions, we assume that the share of tasks allocated to AI is initially small, so that

$$
\text { Exposure to } \mathrm{AI}_{e} \approx \frac{\int_{\mathcal{T}^{A} \cap \mathcal{T}_{e}^{N}} \alpha(x) d x}{\int_{\mathcal{T}_{e}^{N}} \alpha(x) d x}
$$

AI thus reduces unit costs by

$$
d \ln C_{e}=-\left(\int_{\mathcal{T}^{A} \cap \mathcal{T}_{e}^{N}} \alpha(x) d x\right) \cdot \pi_{e}
$$

where

$$
\pi_{e}=\frac{\int_{\mathcal{T}^{A} \cap \mathcal{T}_{e}^{N}} \alpha(x) \cdot\left[\ln \left(w / \gamma^{\ell}(x)\right)-\ln \left(\left(w^{A I}+w^{K}\right) / \gamma^{a}(x)\right)\right]}{\int_{\mathcal{T}^{A} \cap \mathcal{T}_{e}^{N}} \alpha(x) d x}>0
$$

is the average cost savings from substituting AI algorithms for workers in the set of tasks given by $\mathcal{T}^{A}$. 
Then, the change in total cost can be expressed as

$$
d \ln \left(y_{e} \cdot C_{e}\right)=\left(\varepsilon_{e} \cdot \rho_{e}-1\right) \cdot\left(\int_{\mathcal{T}^{A} \cap \mathcal{T}_{e}^{N}} \alpha(x) d x\right) \cdot \pi_{e}
$$

where $\varepsilon_{e}>1$ is the demand elasticity faced by the firm and $\rho_{e}>0$ the establishment's passthrough rate, summarizing how its price responds to the change in total costs; formally,

$$
\rho_{e}=\frac{d \ln p_{e}}{d \ln C_{e}}
$$

Rewriting this expression,

$$
d \ln \left(y_{e} \cdot C_{e}\right)=\left(\varepsilon_{e} \cdot \rho_{e}-1\right) \cdot\left(\int_{\mathcal{T}_{e}^{N}} \alpha(x) d x\right) \cdot \pi_{e} \cdot \text { Exposure to } \mathrm{AI}_{e}
$$

The demand for non-AI workers changes by

$$
\begin{aligned}
d \ln \ell_{e}^{N}= & -\frac{\int_{\mathcal{T}^{A} \cap \mathcal{T}_{e}^{N}} \alpha(x) d x}{\int_{\mathcal{T}_{e}^{N}} \alpha(x) d x}+\left(\varepsilon_{e} \cdot \rho_{e}-1\right) \cdot\left(\int_{\mathcal{T}_{e}^{N}} \alpha(x) d x\right) \cdot \pi_{e} \cdot \text { Exposure to } \mathrm{AI}_{e} \\
& =\left(-1+\left(\varepsilon_{e} \cdot \rho_{e}-1\right) \cdot\left(\int_{\mathcal{T}_{e}^{N}} \alpha(x) d x\right) \cdot \pi_{e}\right) \cdot \text { Exposure to } \mathrm{AI}_{e},
\end{aligned}
$$

and the demand for AI workers changes by

$$
\begin{aligned}
d \ln \ell_{e}^{A I}= & \frac{\int_{\mathcal{T}^{A} \cap \mathcal{T} A I} \alpha(x) d x}{\int_{\mathcal{T}_{e}^{N}} \alpha(x) d x} \cdot \frac{\int_{\mathcal{T}_{e}^{N}} \alpha(x) d x}{\int_{\mathcal{T}_{e}^{A I}} \alpha(x) d x}+\left(\varepsilon_{e} \cdot \rho_{e}-1\right) \cdot\left(\int_{\mathcal{T}_{e}^{N}} \alpha(x) d x\right) \cdot \pi_{e} \cdot \operatorname{Exposure} \text { to } \mathrm{AI}_{e} \\
& =\left(\frac{\int_{\mathcal{T}_{e}^{N}} \alpha(x) d x}{1-\int_{\mathcal{T}_{e}^{N}} \alpha(x) d x}+\left(\varepsilon_{e} \cdot \rho_{e}-1\right) \cdot\left(\int_{\mathcal{T}_{e}^{N}} \alpha(x) d x\right) \cdot \pi_{e}\right) \cdot \text { Exposure to } \mathrm{AI}_{e} .
\end{aligned}
$$




\section{Appendix B: Additional Figures and Tables}


Figure A1: Occupation and Industry Shares in Burning Glass

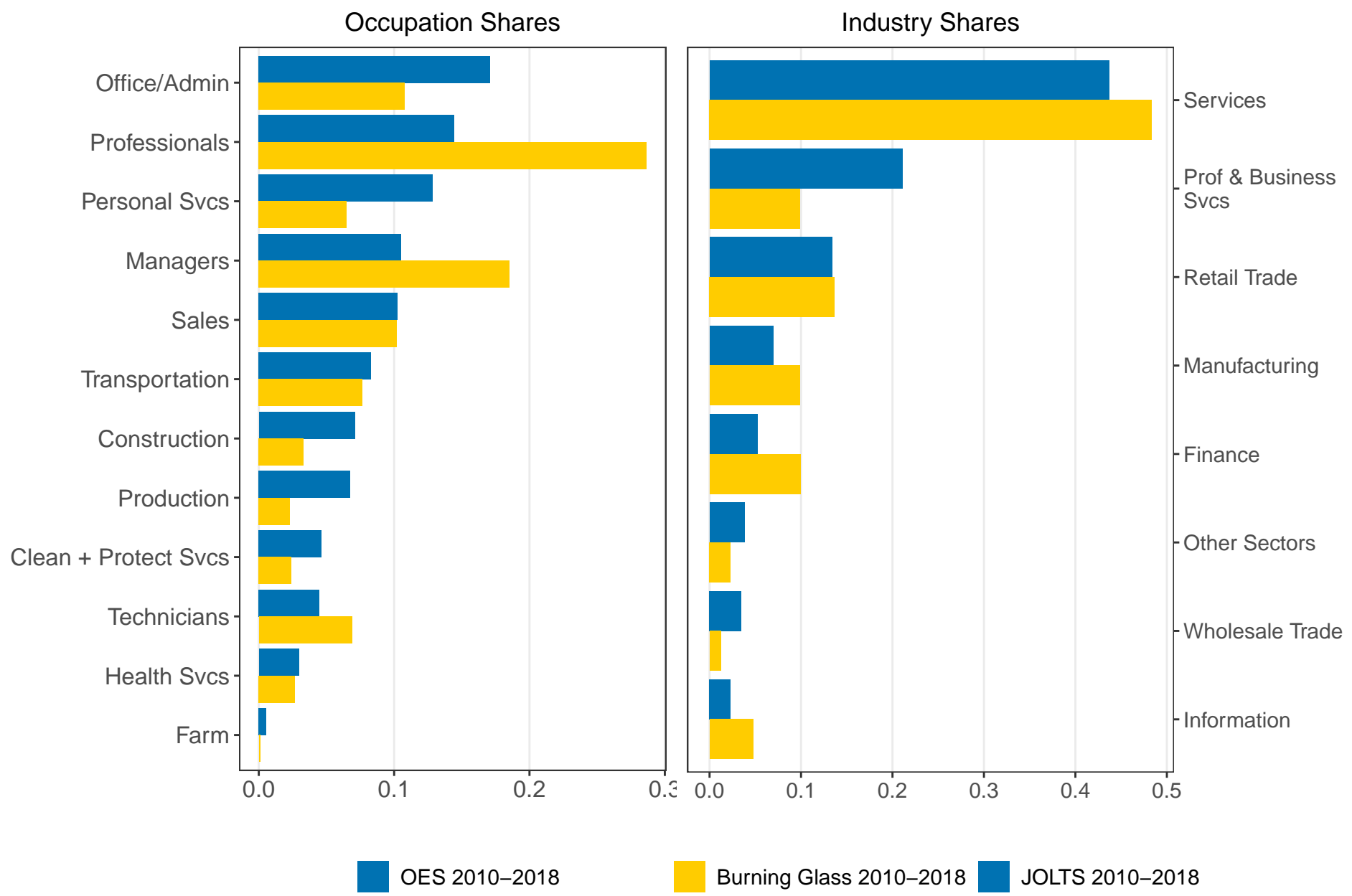

The left panel plots the share of vacancies by broad occupation in the 2010-2018 Burning Glass data, and the share of employment by broad occupation in the 2010-2018 Occupational Employment Statistics. The right panel plots the share of vacancies by broad industry in the 2010-2018 Burning Glass data, and also in JOLTS data. 
TABLE A1: Effects of AI Exposure on Establishment AI Vacancies, Occupation Share Controls

Growth of Establishment AI Vacancies, 2010-2018

(1)

$(2)$

$(3)$

(4)

Panel A: Felten et al Measure of AI Exposure

Establishment AI

Exposure, 2010

Observations

Establishment AI

Exposure, 2010

Observations

Establishment AI

Exposure, 2010

Observations

Covariates:

2010 Vacancy Share

in Broad Occupations

Fixed Effects:

Firm Size Decile

Commuting Zone

3-digit Industry

Firm

$\begin{array}{cccc}10.12 & 9.52 & 7.24 & 13.70 \\ (1.58) & (1.50) & (1.44) & (2.12) \\ 1,075,474 & 1,075,474 & 954,519 & 1,075,474\end{array}$

Panel B: Webb Measure of AI Exposure

$\begin{array}{cccc}-1.55 & -2.81 & -1.75 & -2.06 \\ (1.06) & (0.98) & (0.84) & (1.08) \\ 1,159,789 & 1,159,789 & 1,021,673 & 1,159,789\end{array}$

Panel C: SML Measure of AI Exposure

$\begin{array}{cccc}1.52 & 1.56 & -0.99 & 0.42 \\ (0.95) & (0.89) & (0.91) & (1.26) \\ 1,159,789 & 1,159,789 & 1,021,673 & 1,159,789\end{array}$
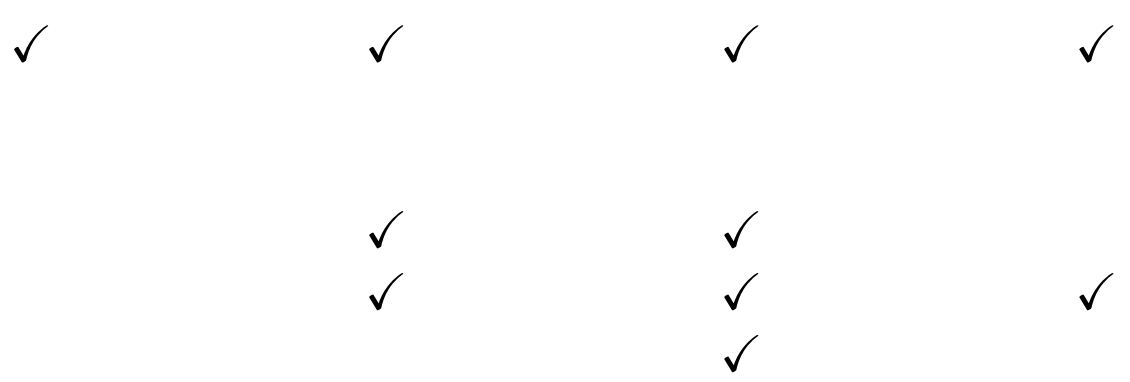

This table presents estimates of the effects of establishment AI exposure on establishment AI vacancy growth. The outcome variable, constructed from Burning Glass data, is the growth rate of AI vacancies between 2010 and 2018 , multiplied by 100. We approximate this growth rate with the change in the inverse hyperbolic sine of the number of vacancies posted by the establishment in 2010-12 and 2016-18. The regressor, establishment AI exposure in 2010, is the mean of occupation AI exposure, over the 6 digit SOC occupations for which the establishment posts vacancies in 2010-2012, weighted by the number of vacancies posted per occupation. Establishment AI exposure is divided by the standard deviation, weighted by $2010-12$ vacancies. In Panel A, the measure of occupation AI exposure is from Felten et al (2019). In Panel B, the measure of occupation AI exposure is SML, from Webb (2020). In Panel C, the measure of occupation AI exposure is from Brynjolfsson et al (2019). The regressor is the establishment's AI exposure. All columns exclude establishments in NAICS sectors 51 (information) and 54 (business services). The covariates included in each model are reported at the bottom of the table. All columns control for the $2010-12$ share of vacancies in each broad occupation, defined in the main text. Column 1 contains only establishment AI exposure. Columns 2 and 3 include fixed effects for the decile of firm total vacancies in 2010-12 to which the establishment's firm belongs. Columns 2-4 include commuting zone fixed effects. Column 3 includes 3-digit NAICS industry fixed effects. Column 4 includes firm fixed effects. In each regression, observations are weighted by total vacancies in 2010-2012. Standard errors are clustered by firm. 
TABle A2: Effect of Establishment AI Exposure on AI Vacancy Growth, Borusyak et al (2021) Standard Errors

Growth of Establishment AI Vacancies, 2010-2018

\begin{tabular}{llll}
\hline$(1)$ & $(2)$ & $(3)$ & $(4)$ \\
\hline
\end{tabular}

Panel A: Felten et al. Measure of AI Exposure

\begin{tabular}{lcccc} 
& \multicolumn{4}{c}{ Panel A: Felten et al. Measure of AI Exposure } \\
Establishment AI & 15.96 & 13.82 & 9.19 & 16.53 \\
Exposure, 2010 & $(1.57)$ & $(1.42)$ & $(1.19)$ & $(2.87)$ \\
Observations & $1,075,474$ & $1,075,474$ & 954,519 & $1,075,474$
\end{tabular}
Observations
$1,075,474$

Panel B: Webb Measure of AI Exposure

Establishment AI
Exposure, 2010

Observations

Establishment AI

Exposure, 2010

Observations

Fixed Effects:

Firm Size Decile

Commuting Zone

3-digit Industry

Firm
6.59

$(2.02)$

5.08

(1.50)

3.21

(1.55)

$1,159,789$

$1,159,789$

$1,021,673$

$1,159,789$

5.91

Panel C: SML Measure of AI Exposure

$\begin{array}{cccc}3.76 & 2.30 & -2.21 & -3.04 \\ (2.72) & (2.09) & (1.67) & (4.21) \\ & & & \\ 1,159,789 & 1,159,789 & 1,021,673 & 1,159,789\end{array}$
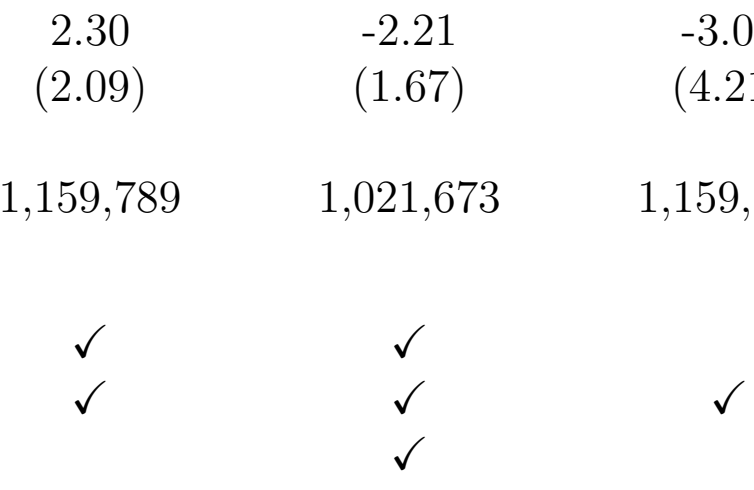

This table presents estimates of the effects of establishment AI exposure on establishment AI vacancy growth, with standard errors from Borusyak et al (2021). The sample is establishments posting vacancies in 2010-12 or 2016-18, outside NAICS sectors 51 (information) and 54 (business services). The outcome variable, constructed from Burning Glass data, is the growth rate of AI vacancies between 2010 and 2018, multiplied by 100. We approximate this growth rate with the change in the inverse hyperbolic sine of the number of vacancies posted by the establishment in 2010-12 and 2016-18. The regressor, establishment AI exposure in 2010, is the standardized mean of occupation AI exposure, over the 6 digit SOC occupations for which the establishment posts vacancies in 2010-2012, weighted by the number of vacancies posted per occupation. In Panel A, the measure of occupation AI exposure is from Felten et al. (2019). In Panel B, the measure of occupation AI exposure is SML, from Brynjolfsson et al. (2019). In Panel C, the measure of occupation AI exposure is from Webb (2020). The covariates included in each model are reported at the bottom of the table. Column 1 contains only establishment AI exposure. Columns 2-3 and 5 include fixed effects for the decile of firm size (defined as the total vacancies posted by an establishment's firm in 2010-2012). Columns 2-6 include commuting zone fixed effects. Columns 3 and 5 include 3-digit NAICS industry fixed effects. Columns 4 and 6 include firm fixed effects. Columns 5 and 6 control for the share of 2010-12 vacancies in each establishment, belonging to the broad occupations of either sales or administration. In each regression, observations are weighted by total establishment vacancies in $2010-2012$. Standard errors are from Borusyak et al (2021) and come from a weighted regression estimated at the 6 digit occupation level, with weights that depend on baseline establishment size. 
TABlE A3: Regression of Establishment AI Growth on Establishment AI Exposure and Occupation Shares

Growth of Establishment AI Vacancies, 2010-2018

(1)

(2)

(3)

(4)

Panel A: Felten et al. Measure of AI Exposure

Adj. Partial R

Squared

Observations

0.0256

0.0157

0.0007

0.0004

107,713

107,713

95,574

107,713

Panel B: Webb Measure of AI Exposure

Adj. Partial R

Squared

Observations

0.0074

0.0055

0.0000

0.0001

116,171

$116,171 \quad 102,263$

116,171

Panel C: SML Measure of AI Exposure

Adj. Partial R

Squared

Observations

0.0014

0.0003

0.0003

0.0014

116,171

116,171

102,263

116,171

Panel D: Felten et al. + SML + Webb Measures

Adj. Partial R

Squared

Observations

0.0278

0.0183

0.0018

0.0028

106,120

106,120

94,221

106,120

Panel E: All Occupation Shares as Regressors

Adj. Partial R

Squared

Observations

0.109

0.083

0.062

0.147

119,625

119,625

105,157

119,625

Fixed Effects:

Firm Size Decile

Commuting Zone

3-digit Industry

Firm
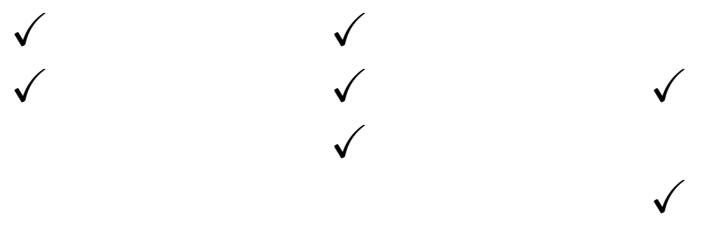

Notes: This table presents the adjusted partial R squared (i.e. the adjusted R squared after "partialling out" the control variables) from regressions of establishment AI exposure on establishment AI vacancy growth or establishment initial occupation composition. The sample starts with establishments posting vacancies in 2010-12 and 2016-18, outside NAICS sectors 51 (information) and 54 (business services). Then we draw a $10 \%$ random sample. The outcome variable, constructed from Burning Glass data, is the growth rate of AI vacancies between 2010 and 2018, multiplied by 100. We approximate this growth rate with the change in the inverse hyperbolic sine of the number of vacancies posted by the establishment in 2010-12 and 2016-18. The regressor, establishment AI exposure in 2010, is the standardized mean of occupation AI exposure, over the 6 digit SOC occupations for which the establishment posts vacancies in 2010-2012, weighted by the number of vacancies posted per occupation. In Panel A, the measure of occupation AI exposure is from Felten et al. (2019). In Panel B, the measure of occupation AI exposure is SML, from Brynjolfsson et al. (2019). In Panel C, the measure of occupation AI exposure is from Webb (2020). Panel D regresses the outcome variable on each of the regressors in Panel A-C. Panel E regresses the outcome variable on the 2010-12 establishment share of vacancies in each 6 digit SOC occupation. The covariates included in each model are reported at the bottom of the table. In each regression, observations are weighted by total establishment vacancies in 2010-2012. Standard errors are clustered by firm. 
TABle A4: Effects of 2007 AI Exposure on Establishment AI Vacancy Growth, 2010-2018

Growth of Establishment AI Vacancies, 2010-2018

(1) (2) (3) (4) (5)

(6)

Panel A: Felten et al. Measure of AI Exposure

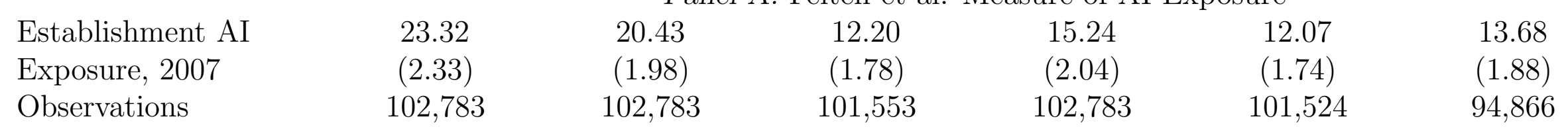

Observations

102,783

$(2.04)$

101,524

94,866

Panel B: Webb Measure of AI Exposure

Establishment AI
Exposure, 2007
Observations

Establishment AI

Exposure, 2007

$\infty \quad$ Observations

$\begin{array}{cc}8.87 & 6.97 \\ (1.71) & (1.54) \\ 106,022 & 106,022\end{array}$

\subsection{6}

(1.99)

106,022

4.49
$(1.33)$
104,719

5.04

(1.39)

106,022

1.92

(1.30)

104,688

Panel C: SML Measure of AI Exposure

5.44
$(1.78)$
106,022

\section{$-3.39$}

(1.82)

106,022
1.78

104,688

Covariates: Share of

Vacancies in sales \&

admin, 2010

Fixed Effects:

Firm Size Decile

Commuting Zone

3-digit Industry

Firm
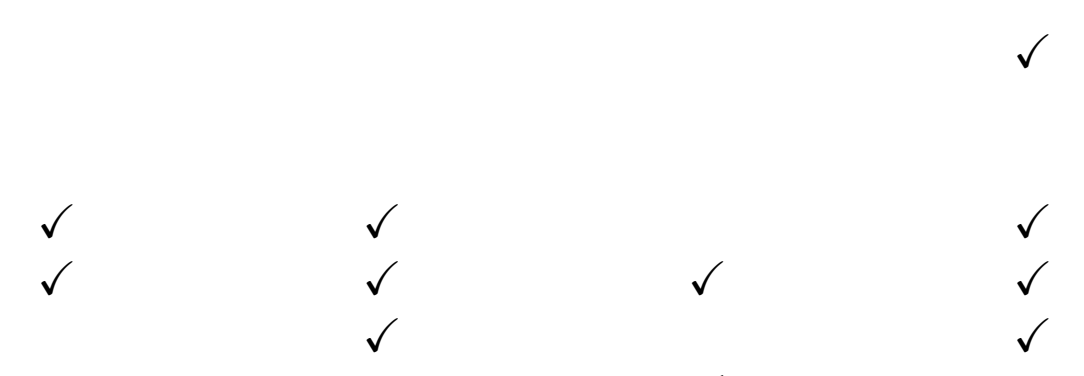

97,919

This table presents estimates of the effects of establishment AI exposure on establishment AI vacancy growth. The sample is establishments posting vacancies in 2010-12 and 2016-18, outside NAICS sectors 51 (information) and 54 (business services). The outcome variable, constructed from Burning Glass data, is the growth rate of AI vacancies between 2010 and 2018, multiplied by 100. We approximate this growth rate with the change in the inverse hyperbolic sine of the number of vacancies posted by the establishment in 2010-12 and 2016-18. The regressor, establishment AI exposure in 2007, is the mean of occupation AI exposure, over the 6 digit SOC occupations for which the establishment posts vacancies in 2007, weighted by the number of vacancies posted per occupation. Establishment AI exposure is divided by the standard deviation, weighted by 2010-12 vacancies. In Panel A, the measure of occupation AI exposure is from Felten et al. (2019). In Panel B, the measure of occupation AI exposure is SML, from Brynjolfsson et al. (2019). In Panel C, the measure of occupation AI exposure is from Webb (2020). The covariates included in each model are reported at the bottom of the table. Column 1 contains only establishment AI exposure. Columns 2-3 and 5 include fixed effects for the decile of firm total vacancies in 2010-12 to which the establishment's firm belongs. Columns 2-6 include commuting zone fixed effects. Columns 3 and 5 include 3 digit NAICS industry fixed effects. Columns 4 and 6 include firm fixed effects. Columns 5 and 6 control for the share of 2010-12 vacancies in each establishment, belonging to the broad occupations of either sales or administration. In each regression, observations are weighted by total establishment vacancies in $2010-2012$. Standard errors are clustered by firm. 
TABle A5: Effects of AI Exposure on Establishment Broad AI Vacancy Growth, 2010-2018

Growth of Establishment Broad AI Vacancies, 2010-2018

(1)

(2)

(5)

(6)

\begin{tabular}{|c|c|c|c|c|c|c|}
\hline & & Pant & Felten et al & sure of AI & sure & \\
\hline Establishment AI & 20.05 & 17.17 & 10.96 & 18.72 & 11.50 & 18.80 \\
\hline Exposure, 2010 & $(1.77)$ & $(1.49)$ & $(1.22)$ & (1.99) & $(1.22)$ & $(1.95)$ \\
\hline Observations & $1,075,474$ & $1,075,474$ & 954,519 & $1,075,474$ & 954,519 & $1,075,474$ \\
\hline & & & B: Webb $\mathrm{N}$ & e of AI Ex & & \\
\hline Establishment AI & 8.47 & 6.43 & 3.72 & 7.38 & 0.99 & 2.53 \\
\hline & & & $C: \mathrm{SML} \mathrm{M}$ & e of AI Ex. & & \\
\hline Establishment AI & 4.14 & 2.18 & -2.22 & -2.90 & 1.92 & 4.90 \\
\hline Exposure, 2010 & $(1.43)$ & $(1.27)$ & $(0.89)$ & $(1.33)$ & $(0.88)$ & $(1.34)$ \\
\hline Observations & $1,159,789$ & $1,159,789$ & $1,021,673$ & $1,159,789$ & $1,021,673$ & $1,159,789$ \\
\hline Fixed Effects: & & & & & & \\
\hline Firm Size Decile & & $\checkmark$ & $\checkmark$ & & $\checkmark$ & \\
\hline Commuting Zone & & $\checkmark$ & $\checkmark$ & $\checkmark$ & $\checkmark$ & $\checkmark$ \\
\hline 3-digit Industry & & & $\checkmark$ & & $\checkmark$ & \\
\hline Firm & & & & $\checkmark$ & & $\checkmark$ \\
\hline
\end{tabular}

Observations

\section{Establishment AI \\ Exposure, 2010}

Covariates: Share of

Vacancies in Sales,

Admin. in 2010

Fixed Effects:

Firm Size Decile

igit Industry

Firm
Exposure, 2010
$1,075,474$
$1,075,474$
0.99

$(0.83)$

$(1.28)$

$1,021,673$

$$
-2.90
$$

$(0.88)$

$1,021,673$

This table presents estimates of the effects of establishment AI exposure on establishment Broad AI vacancy growth. The sample is establishments posting vacancies in 2010-12 or 2016-18, outside NAICS sectors 51 (information) and 54 (business services). Broad AI is defined in the main text, as vacancies that post skills in the skill clusters "Artificial Intelligence" or "Machine Learning". The outcome variable, constructed from Burning Glass data, is the growth rate of AI vacancies between 2010 and 2018, multiplied by 100. We approximate this growth rate with the change in the inverse hyperbolic sine of the number of vacancies posted by the establishment in 2010-12 and 2016-18. The regressor, establishment AI exposure in 2010, is the mean of occupation AI exposure, over the 6 digit SOC occupations for which the establishment posts vacancies in 2010-2012, weighted by the number of vacancies posted per occupation. Establishment AI exposure is divided by the standard deviation, weighted by 2010-12 vacancies. In Panel A, the measure of occupation AI exposure is from Felten et al. (2019). In Panel B, the measure of occupation AI exposure is SML, from Brynjolfsson et al. (2019). In Panel C, the measure of occupation AI exposure is from Webb (2020). The covariates included in each model are reported at the bottom of the table. Column 1 contains only establishment AI exposure. Columns 2-3 and 5 include fixed effects for the decile of firm total vacancies in 2010-12 to which the establishment's firm belongs. Columns 2-6 include commuting zone fixed effects. Columns 3 and 5 include 3 digit NAICS industry fixed effects. Columns 4 and 6 include firm fixed effects. Columns 5 and 6 control for the share of 2010-12 vacancies in each establishment, belonging to the broad occupations of either sales or administration. In each regression, observations are weighted by total establishment vacancies in 2010-2012. Standard errors are clustered by firm. 
TABLE A6: Effects of AI Exposure on Establishment AI Share Change, 2010-2018

Change in Establishment Share of AI Vacancies, 2010-2018

(1)

(2)

(3)

(4)

(5)

(6)

Panel A: Felten et al. Measure of AI Exposure
Establishment A
Exposure, 2010
Observations

Establishment AI
Exposure, 2010
Observations

Establishment AI

Exposure, 2010

0.29
$(0.03)$
341,525

355,529

0.05

$(0.02)$

355,529
0.26
$(0.02)$
341,525

0.20
$(0.02)$
324,901

0.20

324,901

(0.02)

341,525
0.18

341,525
0.22

324,901

Panel B: Webb Measure of AI Exposure

$\begin{array}{cccc}0.22 & 0.14 & 0.11 & 0.12 \\ (0.03) & (0.02) & (0.02) & (0.02) \\ 355,529 & 337,758 & 355,529 & 337,758\end{array}$

0.05

Panel C: SML Measure of AI Exposure

$\begin{array}{cccc}0.03 & -0.06 & -0.08 & 0.04 \\ (0.02) & (0.02) & (0.02) & (0.02) \\ 355,529 & 337,758 & 355,529 & 337,758\end{array}$

0.05

355,529
337,758
Covariates: Share of

Vacancies in Sales,

Admin. in 2010

Fixed Effects:

Firm Size Decile

Commuting Zone

3-digit Industry

Firm
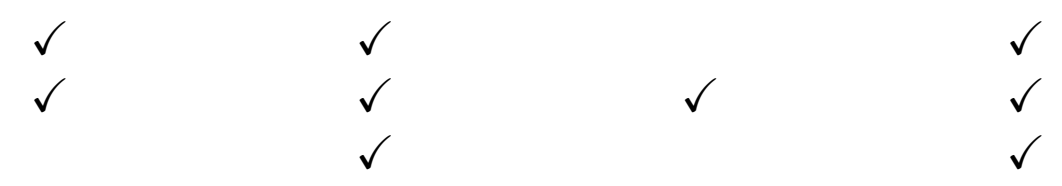

This table presents estimates of the effects of establishment AI exposure on the change in the share of AI vacancies. The outcome variable, constructed from Burning Glass data, is the change in the share of AI vacancies between 2010 and 2018, multiplied by 100. The shares are the ratio of AI vacancies to total vacancies in 2010-12 and 2016-2018. The regressor, establishment AI exposure in 2010, is the mean of occupation AI exposure, over the 6 digit SOC occupations for which the establishment posts vacancies in 2010-2012, weighted by the number of vacancies posted per occupation. Establishment AI exposure is divided by the standard deviation, weighted by 2010-12 vacancies. In Panel A, the measure of occupation AI exposure is from Felten et al. (2019). In Panel B, the measure of occupation AI exposure is SML, from Brynjolfsson et al. (2019). In Panel C, the measure of occupation AI exposure is from Webb (2020). All columns exclude establishments in NAICS sectors 51 (information) and 54 (business services). The covariates included in each model are reported at the bottom of the table. Column 1 contains only establishment AI exposure. Columns 2-3 and 5 include fixed effects for the decile of firm total vacancies in 2010-12 to which the establishment's firm belongs. Columns 2-6 include commuting zone fixed effects. Columns 3 and 5 include 3-digit NAICS industry fixed effects. Columns 4 and 6 include firm fixed effects. Columns 5 and 6 control for the share of 2010-12 vacancies in each establishment, belonging to the broad occupations of either sales or administration. In each regression, observations are weighted by total establishment vacancies in 2010-2012. Standard errors are clustered by firm. 
TABLE A7: Effects of AI Exposure on Firm AI Vacancy Growth, 2010-2018

\begin{tabular}{|c|c|c|c|c|c|c|}
\hline & \multicolumn{2}{|c|}{$\begin{array}{l}\text { Firm AI Vacancy Growth, } \\
\text { 2010-2018 }\end{array}$} & \multicolumn{2}{|c|}{$\begin{array}{l}\text { Firm Mean Establishment } \\
\text { AI Growth, 2010-2018 }\end{array}$} & \multicolumn{2}{|c|}{$\begin{array}{c}\text { Firm AI Vacancy Share } \\
\text { Change, 2010-2018 }\end{array}$} \\
\hline & (1) & $(2)$ & (3) & (4) & $(5)$ & (6) \\
\hline & \multicolumn{6}{|c|}{ Panel A: Felten et al. Measure of AI Exposure } \\
\hline Firm AI Exposure, & -3.77 & -0.49 & 13.60 & 8.01 & 0.29 & 0.27 \\
\hline 2010 & $(4.81)$ & $(4.13)$ & $(1.85)$ & $(1.49)$ & $(0.03)$ & $(0.05)$ \\
\hline \multirow[t]{2}{*}{ Observations } & 401,399 & 320,628 & 401,399 & 320,628 & 110,390 & 99,109 \\
\hline & \multicolumn{6}{|c|}{ Panel B: Webb Measure of AI Exposure } \\
\hline Firm AI Exposure, & -6.83 & -7.02 & 5.29 & 0.66 & 0.22 & 0.14 \\
\hline 2010 & $(4.23)$ & $(4.86)$ & $(1.25)$ & $(1.10)$ & $(0.04)$ & $(0.02)$ \\
\hline \multirow[t]{2}{*}{ Observations } & 431,195 & 339,512 & 431,195 & 339,512 & 114,975 & 102,917 \\
\hline & \multicolumn{6}{|c|}{ Panel C: SML Measure of AI Exposure } \\
\hline Firm AI Exposure, & 6.01 & 3.44 & 4.96 & 1.50 & 0.11 & 0.06 \\
\hline 2010 & $(4.17)$ & $(3.63)$ & $(1.59)$ & $(1.08)$ & $(0.03)$ & $(0.04)$ \\
\hline Observations & 431,195 & 339,512 & 431,195 & 339,512 & 114,975 & 102,917 \\
\hline Covariates: Share of & & & & & & \\
\hline $\begin{array}{l}\text { Vacancies in sales \& } \\
\text { admin, } 2010\end{array}$ & & $\checkmark$ & & $\checkmark$ & & $\checkmark$ \\
\hline Fixed Effects: & & & & & & \\
\hline Firm Size Decile & & $\checkmark$ & & $\checkmark$ & & $\checkmark$ \\
\hline 3 digit Industry & & $\checkmark$ & & $\checkmark$ & & $\checkmark$ \\
\hline
\end{tabular}

This table presents estimates of the effects of firm AI exposure on firm AI vacancy growth. In the first two columns, the outcome variable, constructed from Burning Glass data, is the change in the inverse hyperbolic sine of AI vacancies posted by the firm, between 2010-12 and 2016-18, multiplied by 100. In the middle two columns, the outcome variable is the firm mean of the change in the inverse hyperbolic sine of AI vacancies posted by each establishment in the firm, between 2010-12 and 2016-18, multiplied by 100. The firm mean is weighted by vacancies in each establishment in 2010-2012. In the last two columns, the outcome variable is the change in the ratio of AI vacancies to total vacancies between 2010-12 and 2016-2018, multiplied by 100. The regressor, firm AI exposure in 2010, is the mean of occupation AI exposure, over the 6 digit SOC occupations for which the firm posts vacancies in 2010-2012, weighted by the number of vacancies posted per occupation. Firm AI exposure is divided by the standard deviation, weighted by 2010-12 vacancies in the firm. In Panel A, the measure of occupation AI exposure is from Felten et al. (2019). In Panel B, the measure of occupation AI exposure is SML, from Brynjolfsson et al. (2019). In Panel C, the measure of occupation AI exposure is from Webb (2020). All columns exclude establishments in NAICS sectors 51 (information) and 54 (business services). The covariates included in each model are reported at the bottom of the table. Columns 1, 3 and 5 contains only firm AI exposure. Columns 2, 4 and 6 include fixed effects for the decile of firm total vacancies in 2010-12, 3-digit NAICS industry fixed effects, and controls for the share of 2010-12 vacancies in each firm, belonging to the broad occupations of either sales or administration. In each regression, observations are weighted by total firm vacancies in 2010-2012. Standard errors are clustered by firm. 
TABlE A8: Effects of AI Exposure on Establishment Negative Skill Change, 2010-2018

\begin{tabular}{|c|c|c|c|c|c|c|}
\hline & \multicolumn{6}{|c|}{ Establishment Negative Skill Change, 2010-2018 } \\
\hline & $(1)$ & $(2)$ & $(3)$ & $(4)$ & $(5)$ & (6) \\
\hline & \multicolumn{6}{|c|}{ Panel A: Felten et al. Measure of AI Exposure } \\
\hline Establishment AI & 0.59 & 0.57 & 0.34 & 0.24 & 0.46 & 0.35 \\
\hline Exposure, 2010 & $(0.09)$ & $(0.09)$ & $(0.07)$ & $(0.03)$ & $(0.09)$ & $(0.04)$ \\
\hline Estab. Software & 0.24 & 0.23 & 0.06 & -0.04 & 0.05 & -0.05 \\
\hline Exposure & $(0.11)$ & $(0.11)$ & $(0.07)$ & $(0.03)$ & $(0.08)$ & $(0.03)$ \\
\hline \multirow[t]{2}{*}{ Observations } & 353,107 & 353,107 & 335,589 & 353,107 & 335,589 & 353,107 \\
\hline & \multicolumn{6}{|c|}{ Panel B: Webb Measure of AI Exposure } \\
\hline Establishment AI & 0.95 & 0.92 & 0.80 & 0.45 & 0.99 & 0.56 \\
\hline Exposure, 2010 & $(0.13)$ & $(0.12)$ & $(0.10)$ & $(0.05)$ & $(0.14)$ & $(0.05)$ \\
\hline Estab. Software & -0.50 & -0.49 & -0.52 & -0.39 & -0.49 & -0.38 \\
\hline Exposure & $(0.11)$ & $(0.11)$ & $(0.10)$ & $(0.04)$ & $(0.09)$ & $(0.05)$ \\
\hline \multirow[t]{2}{*}{ Observations } & 353,107 & 353,107 & 335,589 & 353,107 & 335,589 & 353,107 \\
\hline & \multicolumn{6}{|c|}{ Panel C: SML Measure of AI Exposure } \\
\hline Establishment AI & 0.59 & 0.57 & 0.34 & 0.24 & 0.46 & 0.35 \\
\hline Exposure, 2010 & $(0.09)$ & $(0.09)$ & $(0.07)$ & $(0.03)$ & $(0.09)$ & $(0.04)$ \\
\hline Estab. Software & 0.24 & 0.23 & 0.06 & -0.04 & 0.05 & -0.05 \\
\hline Exposure & $(0.11)$ & $(0.11)$ & $(0.07)$ & $(0.03)$ & $(0.08)$ & $(0.03)$ \\
\hline Observations & 353,107 & 353,107 & 335,589 & 353,107 & 335,589 & 353,107 \\
\hline \multicolumn{7}{|l|}{ Covariates: } \\
\hline $\begin{array}{l}\text { Share of Vacancies in } \\
\text { sales \& admin, } 2010 \\
\text { Fixed Effects: }\end{array}$ & & & & & $\checkmark$ & $\checkmark$ \\
\hline Firm Size Decile & & $\checkmark$ & $\checkmark$ & & $\checkmark$ & \\
\hline Commuting Zone & & $\checkmark$ & $\checkmark$ & $\checkmark$ & $\checkmark$ & $\checkmark$ \\
\hline 3-digit Industry & & & $\checkmark$ & & $\checkmark$ & \\
\hline Firm & & & & $\checkmark$ & & $\checkmark$ \\
\hline
\end{tabular}

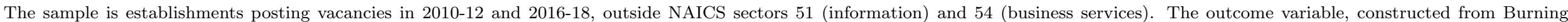

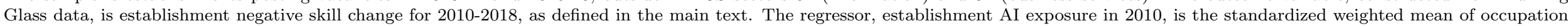

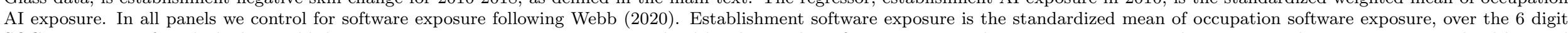

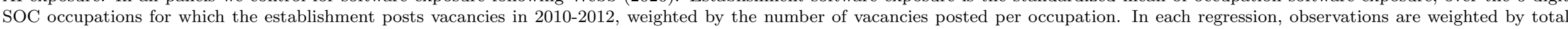
establishment vacancies in 2010-2012. Standard errors are clustered by firm. 
TABle A9: Effects of AI Exposure on Establishment Positive Skill Change, 2010-2018

\begin{tabular}{|c|c|c|c|c|c|c|}
\hline & \multicolumn{6}{|c|}{ Establishment Positive Skill Change, 2010-2018 } \\
\hline & (1) & $(2)$ & $(3)$ & $(4)$ & $(5)$ & (6) \\
\hline & \multicolumn{6}{|c|}{ Panel A: Felten et al. Measure of AI Exposure } \\
\hline Establishment AI & 0.96 & 0.95 & 0.59 & 0.01 & 0.65 & 0.07 \\
\hline Exposure, 2010 & $(0.08)$ & $(0.08)$ & $(0.09)$ & $(0.04)$ & $(0.09)$ & $(0.04)$ \\
\hline Estab. Software & 0.18 & 0.17 & 0.09 & -0.05 & 0.21 & 0.05 \\
\hline Exposure & $(0.09)$ & $(0.09)$ & $(0.08)$ & $(0.03)$ & $(0.07)$ & $(0.04)$ \\
\hline \multirow[t]{2}{*}{ Observations } & 336,425 & 336,425 & 320,208 & 336,425 & 320,208 & 336,425 \\
\hline & \multicolumn{6}{|c|}{ Panel B: Webb Measure of AI Exposure } \\
\hline Establishment AI & 1.03 & 1.01 & 0.41 & 0.04 & 0.54 & 0.15 \\
\hline Exposure, 2010 & $(0.11)$ & $(0.10)$ & $(0.08)$ & $(0.04)$ & $(0.09)$ & $(0.04)$ \\
\hline Estab. Software & -0.54 & -0.52 & -0.22 & -0.08 & -0.17 & -0.03 \\
\hline Exposure & $(0.11)$ & $(0.11)$ & $(0.09)$ & $(0.04)$ & $(0.08)$ & $(0.04)$ \\
\hline \multirow[t]{2}{*}{ Observations } & 353,107 & 353,107 & 335,589 & 353,107 & 335,589 & 353,107 \\
\hline & \multicolumn{6}{|c|}{ Panel C: SML Measure of AI Exposure } \\
\hline Establishment AI & 0.68 & 0.65 & 0.21 & 0.09 & 0.26 & 0.03 \\
\hline Exposure, 2010 & $(0.09)$ & $(0.08)$ & $(0.09)$ & $(0.04)$ & $(0.09)$ & $(0.04)$ \\
\hline Estab. Software & 0.29 & 0.28 & 0.09 & -0.02 & 0.12 & 0.05 \\
\hline Exposure & $(0.08)$ & $(0.08)$ & $(0.08)$ & $(0.03)$ & $(0.07)$ & $(0.03)$ \\
\hline Observations & 353,107 & 353,107 & 335,589 & 353,107 & 335,589 & 353,107 \\
\hline \multicolumn{7}{|l|}{ Covariates: } \\
\hline $\begin{array}{l}\text { Share of Vacancies in } \\
\text { sales \& admin, } 2010\end{array}$ & & & & & $\checkmark$ & $\checkmark$ \\
\hline \multicolumn{7}{|l|}{ Fixed Effects: } \\
\hline Firm Size Decile & & $\checkmark$ & $\checkmark$ & & $\checkmark$ & \\
\hline Commuting Zone & & $\checkmark$ & $\checkmark$ & $\checkmark$ & $\checkmark$ & $\checkmark$ \\
\hline 3-digit Industry & & & $\checkmark$ & & $\checkmark$ & \\
\hline Firm & & & & $\checkmark$ & & $\checkmark$ \\
\hline
\end{tabular}

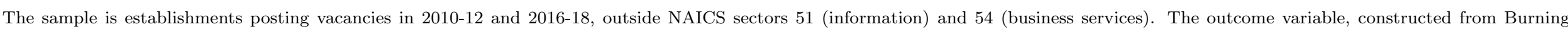

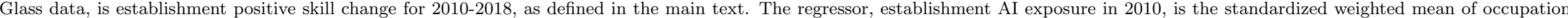

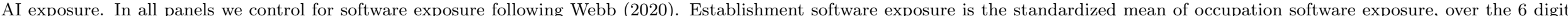

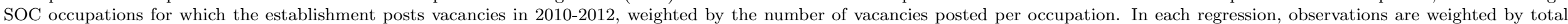
establishment vacancies in 2010-2012. Standard errors are clustered by firm. 
TABLE A10: Effects of AI Exposure on Establishment Total Vacancy Growth, 2014-2018

\begin{tabular}{|c|c|c|c|c|}
\hline & \multicolumn{4}{|c|}{ Growth of Establishment Total Vacancies, 2014-2018 } \\
\hline & (1) & $(2)$ & $(3)$ & $(4)$ \\
\hline & \multicolumn{4}{|c|}{ Panel A: Felten et al. Measure of AI Exposure } \\
\hline Establishment AI & -11.80 & -11.24 & -10.51 & -5.13 \\
\hline Exposure, 2010 & $(3.80)$ & $(2.93)$ & $(2.81)$ & $(1.02)$ \\
\hline \multirow[t]{2}{*}{ Observations } & $1,075,474$ & 954,519 & 954,519 & $1,075,474$ \\
\hline & \multicolumn{4}{|c|}{ Panel B: Webb Measure of AI Exposure } \\
\hline Establishment AI & -9.62 & -4.11 & -2.21 & -1.81 \\
\hline Exposure, 2010 & $(2.42)$ & $(1.83)$ & $(2.16)$ & $(0.71)$ \\
\hline \multirow[t]{2}{*}{ Observations } & $1,159,789$ & $1,021,673$ & $1,021,673$ & $1,159,789$ \\
\hline & \multicolumn{4}{|c|}{ Panel C: SML Measure of AI Exposure } \\
\hline Establishment AI & 3.32 & 0.85 & -0.57 & -0.99 \\
\hline Exposure, 2010 & $(2.09)$ & $(2.30)$ & $(2.75)$ & $(0.92)$ \\
\hline Observations & $1,159,789$ & $1,021,673$ & $1,021,673$ & $1,159,789$ \\
\hline \multicolumn{5}{|l|}{ Covariates: Share of } \\
\hline Vacancies in Sales, & & & $\checkmark$ & \\
\hline \multicolumn{5}{|l|}{ Admin. in 2010} \\
\hline \multicolumn{5}{|l|}{ Fixed Effects: } \\
\hline Firm Size Decile & & $\checkmark$ & $\checkmark$ & \\
\hline Commuting Zone & & $\checkmark$ & $\checkmark$ & $\checkmark$ \\
\hline 3-digit Industry & & $\checkmark$ & $\checkmark$ & \\
\hline Firm & & & & $\checkmark$ \\
\hline
\end{tabular}

This table presents estimates of the effects of establishment AI exposure on establishment total vacancy growth. The outcome variable, constructed from Burning Glass data, is the growth rate of total vacancies between 2014 and 2018, multiplied by 100. We approximate this growth rate with the change in the inverse hyperbolic sine of the number of vacancies posted by the establishment in 2013-15 and 2016-18. The regressor, establishment AI exposure in 2010, is the mean of occupation AI exposure, over the 6 digit SOC occupations for which the establishment posts vacancies in 2010-2012, weighted by the number of vacancies posted per occupation. Establishment AI exposure is divided by the standard deviation, weighted by 2010-12 vacancies. In Panel A, the measure of occupation AI exposure is from Felten et al. (2019). In Panel B, the measure of occupation AI exposure is SML, from Brynjolfsson et al. (2019). In Panel C, the measure of occupation AI exposure is from Webb (2020). All columns exclude establishments in NAICS sectors 51 (information) and 54 (business services). The covariates included in each model are reported at the bottom of the table. Column 1 contains only establishment AI exposure. Columns 2 and 3 include fixed effects for the decile of firm total vacancies in 2010-12 to which the establishment's firm belongs. Columns 2-4 include commuting zone fixed effects. Columns 2-3 include 3-digit NAICS industry fixed effects. Column 4 includes firm fixed effects. In each regression, observations are weighted by total vacancies in 2010-2012. Standard errors are clustered by firm. 
TABLE A11: Effects of AI Exposure on Implied Establishment Non-AI Employment Growth, 2010-2018

\begin{tabular}{|c|c|c|c|c|c|c|c|c|}
\hline & \multicolumn{8}{|c|}{ Growth of Establishment Non-AI Employment, 2010-2018 } \\
\hline & $(1)$ & $(2)$ & $(3)$ & $(4)$ & $(5)$ & (6) & $(7)$ & $(8)$ \\
\hline & \multicolumn{8}{|c|}{ Panel A: Felten et al. Measure of AI Exposure } \\
\hline Establishment AI & -7.24 & -8.91 & -5.62 & -4.86 & -6.36 & -4.80 & -4.57 & -9.11 \\
\hline Exposure, 2010 & $(4.66)$ & $(4.53)$ & $(3.61)$ & $(1.39)$ & $(3.59)$ & $(1.39)$ & $(5.32)$ & $(2.00)$ \\
\hline \multirow[t]{2}{*}{ Observations } & $1,075,474$ & $1,075,474$ & 954,519 & $1,075,474$ & 954,519 & $1,075,474$ & 324,901 & 341,525 \\
\hline & \multicolumn{8}{|c|}{ Panel B: Webb Measure of AI Exposure } \\
\hline Establishment AI & -12.54 & -13.59 & -7.53 & -0.19 & -12.17 & 0.68 & -7.47 & -1.29 \\
\hline Exposure, 2010 & $(3.52)$ & $(3.49)$ & $(2.48)$ & $(0.81)$ & $(3.25)$ & $(0.93)$ & $(3.66)$ & $(1.37)$ \\
\hline \multirow[t]{2}{*}{ Observations } & $1,159,789$ & $1,159,789$ & $1,021,673$ & $1,159,789$ & $1,021,673$ & $1,159,789$ & 337,758 & 355,529 \\
\hline & \multicolumn{8}{|c|}{ Panel C: SML Measure of AI Exposure } \\
\hline Establishment AI & 2.13 & 1.45 & 1.56 & -1.27 & 4.06 & -2.80 & -0.72 & -2.38 \\
\hline Exposure, 2010 & $(3.13)$ & $(3.04)$ & $(2.58)$ & $(0.94)$ & $(2.83)$ & $(1.05)$ & $(4.36)$ & $(1.47)$ \\
\hline Observations & $1,159,789$ & $1,159,789$ & $1,021,673$ & $1,159,789$ & $1,021,673$ & $1,159,789$ & 337,758 & 355,529 \\
\hline $\begin{array}{l}\text { Sample: Posts in } \\
\text { 2018: }\end{array}$ & & & & & & & $\checkmark$ & $\checkmark$ \\
\hline Covariates: Share of & & & & & & & & \\
\hline $\begin{array}{l}\text { Vacancies in sales \& } \\
\text { admin, } 2010\end{array}$ & & & & & $\checkmark$ & $\checkmark$ & & \\
\hline Fixed Effects: & & & & & & & & \\
\hline Firm Size Decile & & $\checkmark$ & $\checkmark$ & & $\checkmark$ & & $\checkmark$ & \\
\hline Commuting Zone & & $\checkmark$ & $\checkmark$ & $\checkmark$ & $\checkmark$ & $\checkmark$ & $\checkmark$ & \\
\hline 3-digit Industry & & & $\checkmark$ & & $\checkmark$ & & $\checkmark$ & \\
\hline Firm & & & & $\checkmark$ & & $\checkmark$ & & $\checkmark$ \\
\hline
\end{tabular}

This table presents estimates of the effects of establishment AI exposure on implied establishment non-AI employment growth. The outcome variable, constructed from Burning Glass data, is the growth rate of establishment non-AI employment between 2010 and 2018, multiplied by 100. We measure establishment employment growth using the procedure for cumulating vacancies into a stock of employment, as described in the main text. We approximate this growth rate with the change in the inverse hyperbolic sine of the number of vacancies posted by the establishment in 2010-12 and 2016-18. The regressor, establishment AI exposure in 2010, is the mean of occupation AI exposure, over the 6 digit SOC occupations for which the establishment posts vacancies in 2010-2012, weighted by the number of vacancies posted per occupation. Establishment AI exposure is divided by the standard deviation, weighted by 2010-12 vacancies. In Panel A, the measure of occupation AI exposure is from Felten et al. (2019). In Panel B, the measure of occupation AI exposure is SML, from Brynjolfsson et al. (2019). In Panel C, the measure of occupation AI exposure is from Webb (2020). All columns exclude establishments in NAICS sectors 51 (information) and 54 (business services). The final two columns exclude establishments that do not post positive vacancies in 2018. The covariates included in each model are reported at the bottom of the table. Column 1 contains only establishment AI exposure. Columns 2, 3, 5 and 7 include fixed effects for the decile of firm total vacancies in 2010-12 to which the establishment's firm belongs. Columns 2-8 include commuting zone fixed effects. Columns 3, 5 and 7 include 3 -digit NAICS industry fixed effects. Columns 4,6 and 8 include firm fixed effects. Columns 5 and 6 control for the share of 2010-12 vacancies in each establishment, belonging to the broad occupations of either sales or administration. In each regression, observations are weighted by total vacancies in 2010-2012. Standard errors are clustered by firm. 
TABlE A12: Effect of Establishment AI Exposure on Non-AI Vacancy Growth, Borusyak et al (2021) Standard Errors

\begin{tabular}{|c|c|c|c|c|}
\hline & \multicolumn{4}{|c|}{ Growth of Establishment Non-AI Vacancies, 2010-2018 } \\
\hline & (1) & $(2)$ & $(3)$ & $(4)$ \\
\hline & \multicolumn{4}{|c|}{ Panel A: Felten et al. Measure of AI Exposure } \\
\hline Establishment AI & -13.80 & -16.36 & -11.90 & -4.81 \\
\hline Exposure, 2010 & $(4.00)$ & $(4.24)$ & $(6.15)$ & $(2.40)$ \\
\hline \multirow[t]{2}{*}{ Observations } & $1,075,474$ & $1,075,474$ & 954,519 & $1,075,474$ \\
\hline & \multicolumn{4}{|c|}{ Panel B: Webb Measure of AI Exposure } \\
\hline Establishment AI & -17.24 & -18.21 & -6.73 & -2.22 \\
\hline Exposure, 2010 & $(4.44)$ & $(4.97)$ & $(5.41)$ & $(3.33)$ \\
\hline \multirow[t]{2}{*}{ Observations } & $1,159,789$ & $1,159,789$ & $1,021,673$ & $1,159,789$ \\
\hline & \multicolumn{4}{|c|}{ Panel C: SML Measure of AI Exposure } \\
\hline Establishment AI & 7.02 & 5.74 & 2.05 & 0.95 \\
\hline Exposure, 2010 & $(4.56)$ & $(4.53)$ & $(3.64)$ & $(3.74)$ \\
\hline Observations & $1,159,789$ & $1,159,789$ & $1,021,673$ & $1,159,789$ \\
\hline \multicolumn{5}{|l|}{ Fixed Effects: } \\
\hline Firm Size Decile & & $\checkmark$ & $\checkmark$ & \\
\hline Commuting Zone & & $\checkmark$ & $\checkmark$ & $\checkmark$ \\
\hline 3-digit Industry & & & $\checkmark$ & \\
\hline Firm & & & & $\checkmark$ \\
\hline
\end{tabular}

This table presents estimates of the effects of establishment AI exposure on establishment non-AI vacancy growth, with standard errors from Borusyak et al (2021). The sample is establishments posting vacancies in 2010-12 and 2016-18, outside NAICS sectors 51 (information) and 54 (business services). The outcome variable, constructed from Burning Glass data, is the growth rate of non-AI vacancies between 2010 and 2018, multiplied by 100. We approximate this growth rate with the change in the inverse hyperbolic sine of the number of vacancies posted by the establishment in 2010-12 and 2016-18. The regressor, establishment AI exposure in 2010, is the standardized mean of occupation AI exposure, over the 6 digit SOC occupations for which the establishment posts vacancies in 2010-2012, weighted by the number of vacancies posted per occupation. In Panel A, the measure of occupation AI exposure is from Felten et al. (2019). In Panel B, the measure of occupation AI exposure is SML, from Brynjolfsson et al. (2019). In Panel C, the measure of occupation AI exposure is from Webb (2020). The covariates included in each model are reported at the bottom of the table. Column 1 contains only establishment AI exposure. Columns 2 and 3 include fixed effects for the decile of firm size (defined as the total vacancies posted by an establishment's firm in 2010-2012). Columns 2-4 include commuting zone fixed effects. Column 3 includes 3-digit NAICS industry fixed effects. Column 4 includes firm fixed effects. In each regression, observations are weighted by total vacancies in 2010-2012. Standard errors are from Borusyak et al (2021) and come from a weighted regression estimated at the 6 digit occupation level, with weights that depend on baseline establishment size. 
TABle A13: Regression of Establishment Non-AI Vacancy Growth on AI Growth, 2010-2018

\begin{tabular}{|c|c|c|c|c|c|c|c|c|}
\hline & \multicolumn{8}{|c|}{ Growth of Establishment Non-AI Vacancies, 2010-2018 } \\
\hline & \multicolumn{6}{|c|}{ Full Sample } & \multicolumn{2}{|c|}{ Establishments Posting in 2018} \\
\hline & $(1)$ & $(2)$ & $(3)$ & $(4)$ & $(5)$ & (6) & $(7)$ & (8) \\
\hline Establishment AI & 0.67 & 0.66 & 0.67 & 0.29 & 0.67 & 0.30 & 0.40 & 0.19 \\
\hline Growth, 2010-18 & $(0.03)$ & $(0.03)$ & $(0.03)$ & $(0.02)$ & $(0.03)$ & $(0.02)$ & $(0.02)$ & $(0.01)$ \\
\hline Observations & $1,194,154$ & $1,194,154$ & $1,050,500$ & $1,194,154$ & $1,050,500$ & $1,194,154$ & 343,266 & 361,471 \\
\hline \multicolumn{9}{|l|}{ Covariates: Share of } \\
\hline Vacancies in Sales, & & & & & $\checkmark$ & $\checkmark$ & & \\
\hline \multicolumn{9}{|l|}{ Admin. in 2010} \\
\hline \multicolumn{9}{|l|}{ Fixed Effects: } \\
\hline Firm Size Decile & & $\checkmark$ & $\checkmark$ & & $\checkmark$ & & $\checkmark$ & \\
\hline Commuting Zone & & $\checkmark$ & $\checkmark$ & $\checkmark$ & $\checkmark$ & $\checkmark$ & $\checkmark$ & $\checkmark$ \\
\hline 3 digit Industry & & & $\checkmark$ & & $\checkmark$ & & $\checkmark$ & \\
\hline Firm & & & & $\checkmark$ & & $\checkmark$ & & $\checkmark$ \\
\hline
\end{tabular}

This table presents estimates from a regression of of establishment non-AI vacancy growth on establishment AI vacancy growth. The outcome variable, constructed from Burning Glass data, is the growth rate of non-AI vacancies between 2010 and 2018, multiplied by 100. We approximate this growth rate with the change in the inverse hyperbolic sine of the number of vacancies posted by the establishment in 2010-12 and 2016-18. The regressor, establishment AI vacancy growth is the change in the inverse hyperbolic sine of the number of AI vacancies posted by the establishment in 2010-12 and 2016-18. The final two columns exclude establishments that do not post positive vacancies in 2018. The covariates included in each model are reported at the bottom of the table. Column 1 contains only establishment AI exposure. Columns 2, 3, 5 and 7 include fixed effects for the decile of firm size (defined as the total vacancies posted by an establishment's firm in 2010-2012). Columns 2-8 include commuting zone fixed effects. Columns 3,5 and 7 include 3-digit NAICS industry fixed effects. Columns 4, 6 and 8 include firm fixed effects. Columns 5 and 6 control for the share of 2010-12 vacancies in each establishment, belonging to the broad occupations of either sales or administration. In each regression, observations are weighted by total vacancies in 2010-2012. Standard errors are clustered by firm. 
TABLE A14: Effects of AI Exposure on Firm Non-AI Vacancy Growth, 2010-2018

\begin{tabular}{|c|c|c|c|c|c|}
\hline & \multicolumn{5}{|c|}{ Growth of Firm Non-AI Vacancies, 2010-2018 } \\
\hline & (1) & (2) & (3) & (4) & (5) \\
\hline $\begin{array}{l}\text { Firm AI Exposure, } \\
2010 \\
\text { Observations }\end{array}$ & $\begin{array}{c}-14.06 \\
(5.40) \\
401,399\end{array}$ & $\begin{array}{c}\text { nel A: Fe } \\
-12.09 \\
(5.25) \\
401,399\end{array}$ & $\begin{array}{l}\text { al. Measl } \\
-5.94 \\
(8.09) \\
320,628\end{array}$ & $\begin{array}{c}\text { I Exposi } \\
-5.85 \\
(9.54) \\
99,109\end{array}$ & $\begin{array}{c}-6.92 \\
(7.79) \\
320,628\end{array}$ \\
\hline $\begin{array}{l}\text { Firm AI Exposure, } \\
2010 \\
\text { Observations }\end{array}$ & $\begin{array}{c}-23.16 \\
(4.98) \\
431,195\end{array}$ & $\begin{array}{c}\text { Panel B: } \\
-22.74 \\
(4.88) \\
431,195\end{array}$ & $\begin{array}{c}\text { Measure } \\
-4.95 \\
(5.65) \\
339,512\end{array}$ & $\begin{array}{c}\text { Xxposure } \\
0.56 \\
(6.76) \\
102,917\end{array}$ & $\begin{array}{c}-14.39 \\
(6.82) \\
339,512\end{array}$ \\
\hline $\begin{array}{l}\text { Firm AI Exposure, } \\
2010 \\
\text { Observations }\end{array}$ & $\begin{array}{c}7.38 \\
(4.65) \\
431,195\end{array}$ & $\begin{array}{c}\text { Panel C } \\
7.59 \\
(4.49) \\
431,195\end{array}$ & $\begin{array}{c}\text { Measure } \\
3.68 \\
(5.36) \\
339,512\end{array}$ & $\begin{array}{c}\text { xposure } \\
-3.05 \\
(6.00) \\
102,917\end{array}$ & $\begin{array}{c}14.71 \\
(6.72) \\
339,512\end{array}$ \\
\hline $\begin{array}{l}\text { Sample: Posts in } \\
\text { 2018: } \\
\text { Covariates: Share of } \\
\text { Vacancies in sales \& } \\
\text { admin, 2010 } \\
\text { Fixed Effects: } \\
\text { Firm Size Decile } \\
\text { 3-digit Industry }\end{array}$ & & $\checkmark$ & $\begin{array}{l}\checkmark \\
\checkmark\end{array}$ & $\begin{array}{l}\checkmark \\
\checkmark\end{array}$ & $\begin{array}{l}\checkmark \\
\checkmark\end{array}$ \\
\hline
\end{tabular}

This table presents estimates of the effects of firm AI exposure on firm non-AI vacancy growth. The outcome variable, constructed from Burning Glass data, is the change in the inverse hyperbolic sine of non-AI vacancies posted by the firm, between 2010-12 and 2016-18, multiplied by 100. The regressor, firm AI exposure in 2010, is the mean of occupation AI exposure, over the 6 digit SOC occupations for which the firm posts vacancies in 2010-2012, weighted by the number of vacancies posted per occupation. Firm AI exposure is divided by the standard deviation, weighted by 2010-12 vacancies in the firm. In Panel A, the measure of occupation AI exposure is from Felten et al. (2019). In Panel B, the measure of occupation AI exposure is SML, from Brynjolfsson et al. (2019). In Panel C, the measure of occupation AI exposure is from Webb (2020). All columns exclude establishments in NAICS sectors 51 (information) and 54 (business services). The covariates included in each model are reported at the bottom of the table. Column 1 contains only firm AI exposure. Columns 2-5 include fixed effects for the decile of firm total vacancies in 2010-12. Columns 3-5 include 3-digit NAICS industry fixed effects. Column 5 controls for the share of 2010-12 vacancies in each firm, belonging to the broad occupations of either sales or administration. Column 5 restricts the sample to firms with positive vacancies in 2018 . In each regression, observations are weighted by total firm vacancies in 2010-2012. Standard errors are clustered by firm. 
TABLE A15: Effects of AI Exposure on Establishment Non-AI Vacancy Growth, Average Establishment Weights

\begin{tabular}{|c|c|c|c|c|}
\hline & \multicolumn{4}{|c|}{ Growth of Establishment Non-AI Vacancies, 2014-2018 } \\
\hline & (1) & $(2)$ & (3) & $(4)$ \\
\hline & \multicolumn{4}{|c|}{ Panel A: Felten et al. Measure of AI Exposure } \\
\hline Establishment AI & -10.69 & -4.89 & -3.42 & -4.08 \\
\hline Exposure, 2010 & $(2.45)$ & $(2.22)$ & $(2.21)$ & $(1.17)$ \\
\hline \multirow[t]{2}{*}{ Observations } & $1,075,474$ & 954,519 & 954,519 & $1,075,474$ \\
\hline & \multicolumn{4}{|c|}{ Panel B: Webb Measure of AI Exposure } \\
\hline Establishment AI & -2.89 & 1.71 & 3.98 & -1.14 \\
\hline Exposure, 2010 & $(1.94)$ & $(1.85)$ & $(2.09)$ & $(0.94)$ \\
\hline \multirow[t]{2}{*}{ Observations } & $1,159,789$ & $1,021,673$ & $1,021,673$ & $1,159,789$ \\
\hline & \multicolumn{4}{|c|}{ Panel C: SML Measure of AI Exposure } \\
\hline Establishment AI & -2.60 & 1.63 & 3.41 & 0.75 \\
\hline Exposure, 2010 & $(1.90)$ & $(1.77)$ & $(2.09)$ & $(0.85)$ \\
\hline Observations & $1,159,789$ & $1,021,673$ & $1,021,673$ & $1,159,789$ \\
\hline \multicolumn{5}{|l|}{ Covariates: Share of } \\
\hline Vacancies in Sales, & & & $\checkmark$ & \\
\hline \multicolumn{5}{|l|}{ Admin. in 2010} \\
\hline \multicolumn{5}{|l|}{ Fixed Effects: } \\
\hline Firm Size Decile & & $\checkmark$ & $\checkmark$ & \\
\hline Commuting Zone & & $\checkmark$ & $\checkmark$ & $\checkmark$ \\
\hline 3-digit Industry & & $\checkmark$ & $\checkmark$ & \\
\hline Firm & & & & $\checkmark$ \\
\hline
\end{tabular}

This table presents estimates of the effects of establishment AI exposure on establishment non-AI vacancy growth. In each regression, observations are weighted by average vacancies in 2010-2012 and 2016-2018. The outcome variable, constructed from Burning Glass data, is the growth rate of non-AI vacancies between 2010 and 2018, multiplied by 100. We approximate this growth rate with the change in the inverse hyperbolic sine of the number of vacancies posted by the establishment in 2010-12 and 2016-18. The regressor, establishment AI exposure in 2010, is the mean of occupation AI exposure, over the 6 digit SOC occupations for which the establishment posts vacancies in 2010-2012, weighted by the number of vacancies posted per occupation. Establishment AI exposure is divided by the standard deviation, weighted by 2010-12 vacancies. In Panel A, the measure of occupation AI exposure is from Felten et al. (2019). In Panel B, the measure of occupation AI exposure is SML, from Brynjolfsson et al. (2019). In Panel C, the measure of occupation AI exposure is from Webb (2020). All columns exclude establishments in NAICS sectors 51 (information) and 54 (business services). The final two columns exclude establishments that do not post positive vacancies in 2018. The covariates included in each model are reported at the bottom of the table. Column 1 contains only establishment AI exposure. Columns 2, 3, 5 and 7 include fixed effects for the decile of firm total vacancies in 2010-12 to which the establishment's firm belongs. Columns 2-8 include commuting zone fixed effects. Columns 3,5 and 7 include 3 -digit NAICS industry fixed effects. Columns 4, 6 and 8 include firm fixed effects. Columns 5 and 6 control for the share of 2010-12 vacancies in each establishment, belonging to the broad occupations of either sales or administration. Standard errors are clustered by firm. 